Portland State University

PDXScholar

$9-29-2021$

\title{
Building a Biomechanical Model of a Rat Forelimb
}

Joshua Nathaniel Eric Mak

Portland State University

Follow this and additional works at: https://pdxscholar.library.pdx.edu/open_access_etds

Part of the Biomechanical Engineering Commons

Let us know how access to this document benefits you.

\section{Recommended Citation}

Mak, Joshua Nathaniel Eric, "Building a Biomechanical Model of a Rat Forelimb" (2021). Dissertations and Theses. Paper 5819.

https://doi.org/10.15760/etd.7690

This Thesis is brought to you for free and open access. It has been accepted for inclusion in Dissertations and Theses by an authorized administrator of PDXScholar. Please contact us if we can make this document more accessible: pdxscholar@pdx.edu. 
Building a Biomechanical Model of a Rat Forelimb

by

Joshua Nathaniel Eric Mak

A thesis submitted in partial fulfillment of the requirements for the degree of

\author{
Master of Science \\ in \\ Mechanical Engineering
}

Thesis Committee:

Alexander Hunt, Chair

Mark M. Weislogel

Faryar Etesami

Portland State University

2021 


\begin{abstract}
This paper presents a biomechanical model of the rat forelimb to test theories of determining viscoelastic muscle parameters. Several biomechanical models of rat hindlimbs have been developed and have explored the effects of multi-muscle control during locomotion. This paper presents a biomechanical model of the rat forelimb. The forelimb model uses two ball-and-socket joints to model clavicle and scapula movement. A third ball-and-socket joint is used at the shoulder and two hinge joints are used at the elbow and wrist. Scapula motion is further constrained by muscle and spring elements. Each forelimb has 11 degrees of freedom, and 23 Hill-type muscles. The model has been created in Animatlab, which includes both a neural design component and a physics environment. Muscle paths are hand guided to approximate the origin and insertion points necessary to replicate multi-body articulation. Most muscles are represented with a single linear muscle path, except in cases where muscle wrapping was necessary around joints or bones. Explored in this work are multiple methods for setting passive muscle parameters, including the use of scaling heuristics and optimization with experimental data. Stiffness and damping muscle parameters are being validated against kinematic and kinetic data from the rat.
\end{abstract}




\section{Dedication}

I would like to dedicate this work to my wife Heather, who's unwavering support has enabled me to pursue my dreams, and to my children Alexa, Eliana, and Gabriel, who's love and curiosity helped drive me to complete this work. I hope to set this as an example so they too can work hard and follow their passions. 


\section{Acknowledgments}

I would like to thank and acknowledge the support of Dr. Alex Hunt for his guidance and support on this thesis, the Agile and Adaptive Robotics lab, especially Cody Sharzenberger for always providing useful feedback. I would also like to thank Dr. Faryar Etesami and Dr. Mark Weislogel for serving on my thesis committee. Additionally, I would like to thank the members of the International Research Group (IRG4) team, specifically, Dr. Roger Quinn, Dr. Matt Tresch, Dr. Martin Fisher, Dr. Emmanuel Andrada, and Fletcher Young for their support and feedback throughout the project. Furthermore, I would like to thank Dr. Thomas Hancock for his support and feedback. Finally, I would like to thank Portland State University for their support and the National Science Foundation for funding under (NSF) grant for NeuroNex: Communication, Coordination, and Control in Systems (C3NS), NSF NeuroNex 2015317, as well as NSF grant 1943483 . This work is solely my responsibility and does not necessarily represent the official view of the National Science Foundation, or any University. 


\section{Table of Contents}

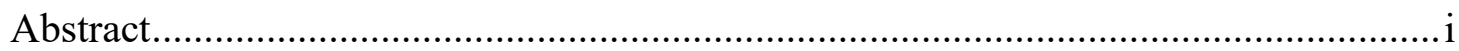

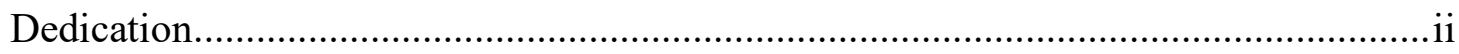

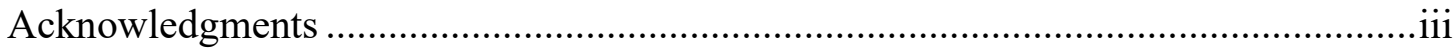

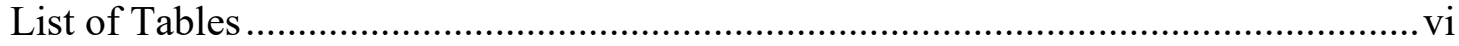

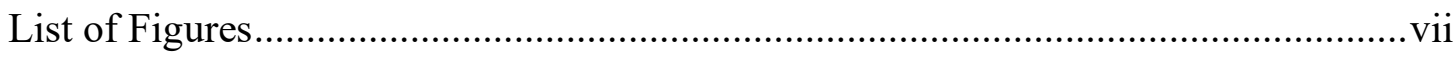

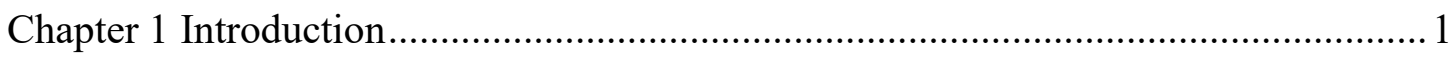

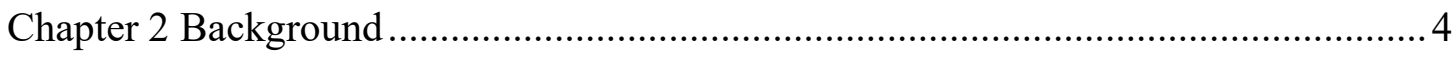

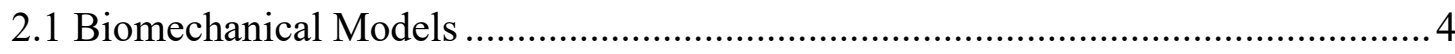

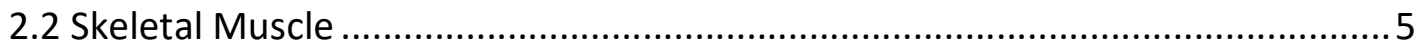

2.3 Hill's Mechanical Model of Skeletal Muscle ....................................................12

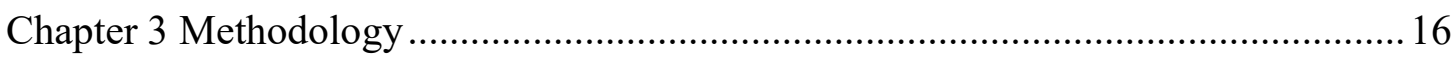

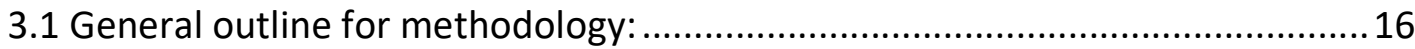

3.2 Determining Rat Forelimb Muscle Architecture..............................................17

3.2.1 Linear Relationships between the dog and rat muscle architecture ...........19

3.2.2 Dog Rat Hindlimb Comparison Using a Proportional Length Method......... 20

3.2.3 Multiple Linear Regression of Separate Clusters Determined by K-Means

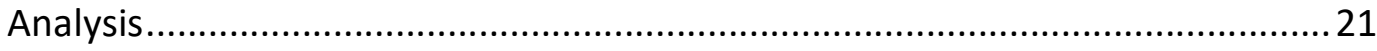

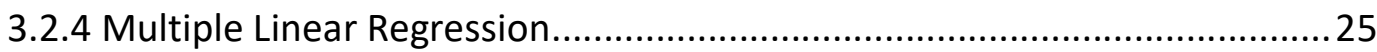

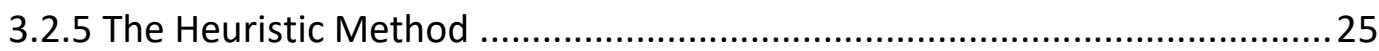

3.2.6 Muscle Fiber Type ................................................................................ 30

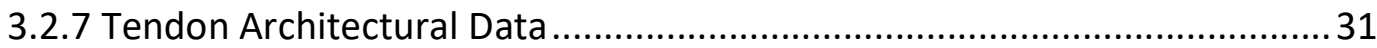

3.3 Viscoelastic Passive and Active Parameters ……………..................................33

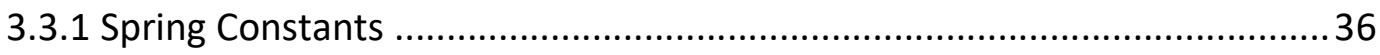

3.3.2 The Active Contractile Component: Specific Muscle Tension ......................37

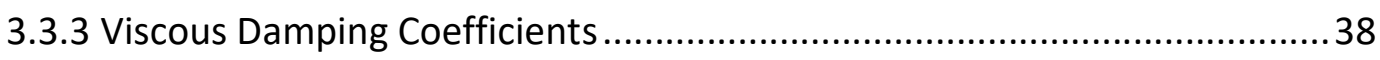

3.3.4 The Muscle Parameter Model.....................................................................4 41

3.4 Building the model in Animatlab ................................................................. 42

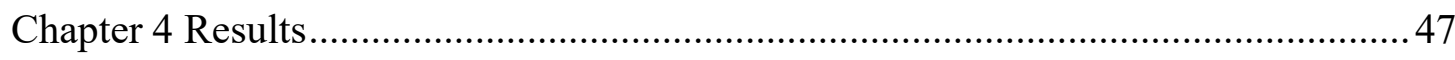

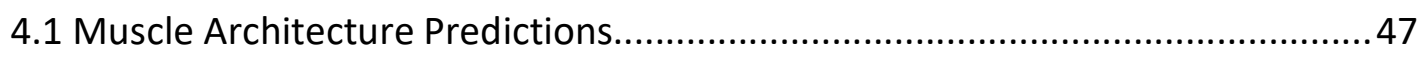


4.1.1 Linear relationships between the dog and rat muscle architecture........... 47

4.1.2 Dog Rat Hindlimb Comparison Using Proportional Lengths .......................47

4.1.3 Multiple Linear Regression of Separate Clusters Determined by K-Means

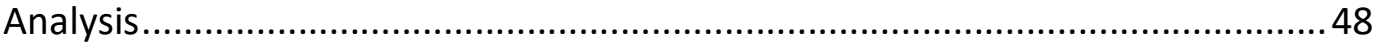

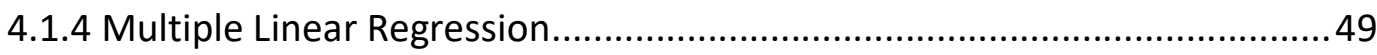

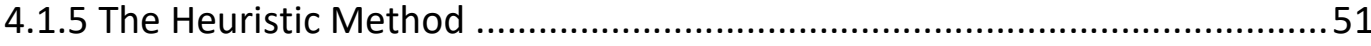

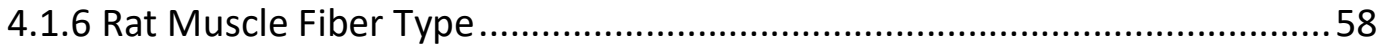

4.1.6 The Rat Forelimb Muscle Architectural Predictions ..................................59

4.1.7 The Tendon Architectural Predictions ....................................................6 60

4.2 The Passive and Active Predicted Muscle Parameters for the Rat Forelimb .....61

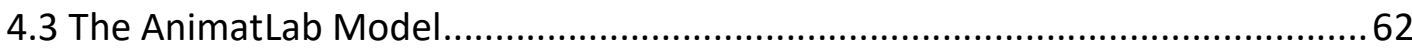

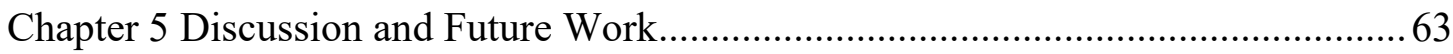

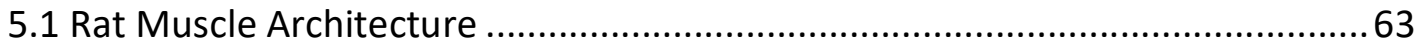

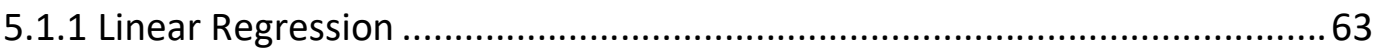

5.1.2 Using the proportional length approach. ................................................63 63

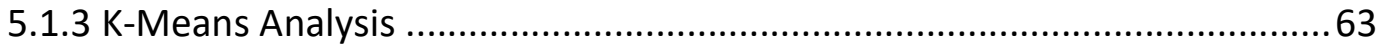

5.1.4 Multiple Linear Regression......................................................................64

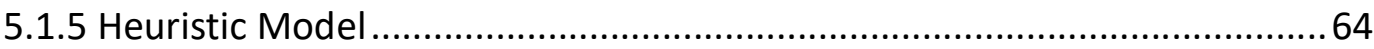

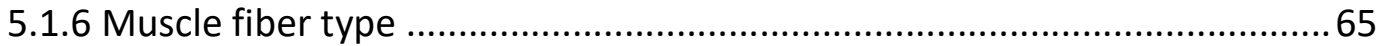

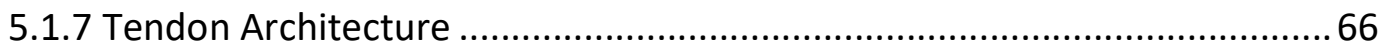

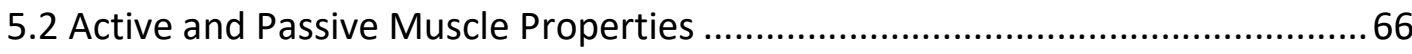

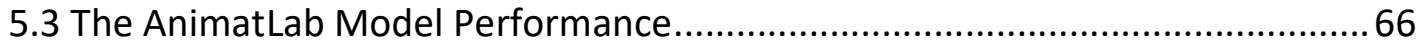

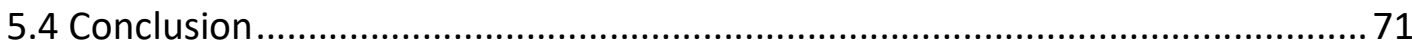

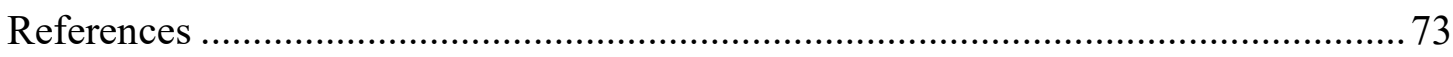

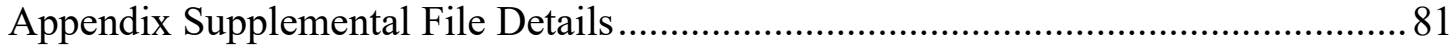




\section{List of Tables}

Table 1. Muscle Fiber Characteristics .................................................................... 11

Table 2. Proportional lengths of the dog and rat hindlimb ......................................20

Table 3. The different $k$-means analyses that were used along with their inputs.........23

Table 4. The results from a $k$-means analysis. DOA $=$ dog only analysis, $R O A=$ rat

only analysis, and DRA = dog-rat analysis......................................................... 23

Table 5. Coefficients used to determine the rat forelimb data. ..................................25

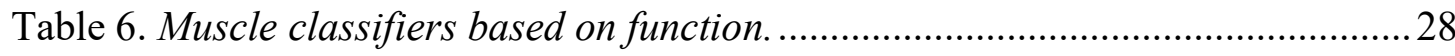

Table 7. Hindlimb classifiers...................................................................................... 28

Table 8. Forelimb muscle classes determined by observation. .................................. 30

Table 9. The mean percent error for each predicted value when compared to the real

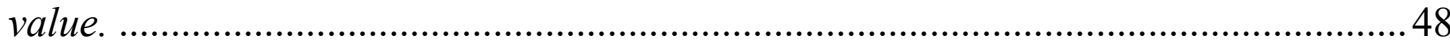

Table 10. The k-means clustered multi-linear regression models used to predict rat muscle architecture. Each row is a different model. ................................................. 49

Table 11. The multi-linear regression models trained on dog and rat hindlimb data

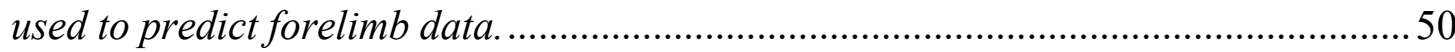

Table 12. Slope intercept equations used. ............................................................. 51

Table 13. Shift necessary for each difference between the linear rat-dog model and the heuristic approach. Values are given in mean \pm standard deviation. .........................53

Table 14. The percent error for each model. Values are represented as mean \pm standard deviation. .................................................................................................... 56

Table 15. The adjustments needed to transition from dog fast-twitch muscle percentage to rat........................................................................................................... 58

Table 16. Forelimb architecture predicted by the heuristic method. ..........................59

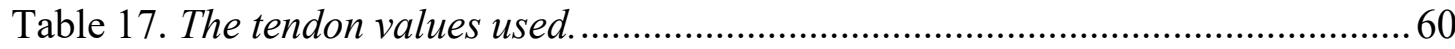

Table 18. The passive and active muscle parameters predicted for the forelimb. .......61 


\section{List of Figures}

Figure 1.1. The simulated rat in AnimatLab................................................................. 3

Figure 2.1. The individual components that make up skeletal muscle. ........................6

Figure 2.2. Sarcomeres are the underlying mechanisms that generate contractile force.

Figure 2.3. The power stroke is the interaction of actin, myosin, and ATP. ................ 8

Figure 2.4. The different types of pennate muscles. .................................................. 10

Figure 2.5. The linear-Hill muscle model............................................................. 14

Figure 3.1. Reconstructing the power law relationship between mass and body weight

for different dog breeds used by Stark with the addition of the rat............................ 18

Figure 3.2. The "Elbow Method" shows how the distortion in the system increase as

the number of clusters used in k-means analysis increase ........................................22

Figure 3.3. The difference between the real and single linear prediction. If the value is

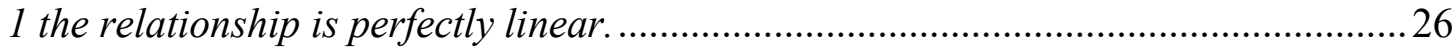

Figure 3.4. The standing posture of a larger dog and a rat. ...................................... 27

Figure 3.5. The comparison of physiological cross-sectional area (PCSA) and

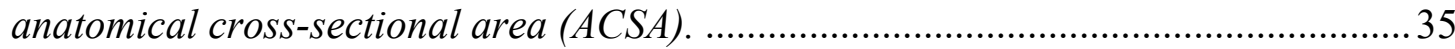

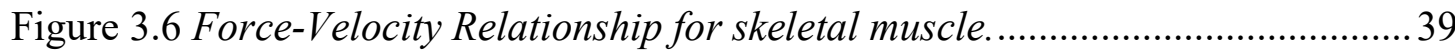

Figure 3.7. The rat forelimb is comprised of three spherical joints and two revolute

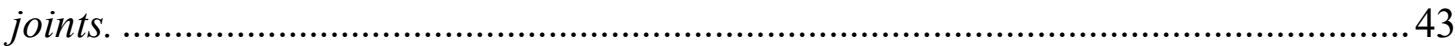

Figure 3.8. The mathematical system describing the movement of the rat forelimb. ... 44

Figure 3.9. The muscles used to manipulate the forelimbs of the rat. Image taken from

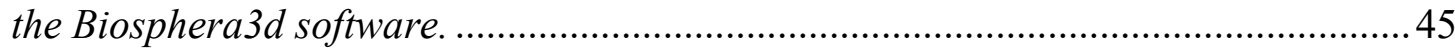

Figure 4.1. Dog-Rat muscle architecture approximate linear relationships............... 47

Figure 4.2. The results from the "Elbow Method" used in python to determine the number of clusters needed for analysis.

Figure 4.3. The predicted forelimb values using the multiple linear regression models.

Figure 4.4. The difference between the real and single linear prediction. If the value is

1 the relationship is perfectly linear. .52

Figure 4.5. The predicted muscle mass values for the rat hindlimb using the heuristic compared to the real rat values.

Figure 4.6. The predicted muscle resting length values for the rat hindlimb using the heuristic compared to the real rat values.

Figure 4.7. The predicted fiber length values for the rat hindlimb using the heuristic compared to the real rat values.

Figure 4.8. The predicted PCSA values for the rat hindlimb using the heuristic

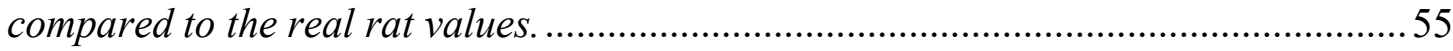
Figure 4.9. The rat forelimb muscle mass predictions from the linear model as well as the heuristic model. 56 
Figure 4.10. The rat forelimb muscle resting length predictions from the linear model

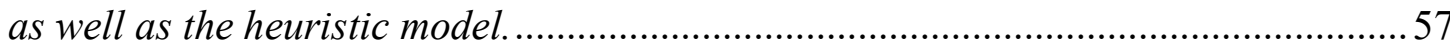
Figure 4.11. The rat forelimb fiber length predictions from the linear model as well as the heuristic model.

Figure 4.12. The rat forelimb PCSA predictions from the linear model as well as the heuristic model.

Figure 4.13. The AnimatLab model of the rat. Blue lines represent the muscle-tendon unit.

Figure 5.1. A screen shot of the video used to validate the AnimatLab model, showing

the initial conditions for test 1 .

Figure 5.2. A screen shot of the video used to validate the AnimatLab model, showing the initial conditions for test 2 . .68

Figure 5.3. The image on the left is of the AnimatLab model and the image on the right is an anesthetized rat. The red arrows indicate where the limb begins to distinguish itself from the main body and the yellow dotted arrow indicated a possible spring force exerted on the forelimb by the skin.... 


\section{Chapter 1 Introduction}

Balancing is an integral part of legged locomotion, and while animals can achieve this effortlessly, similar accomplishments for modern robots prove elusive. Understanding how animals are able to maintain stable positions is still an area of considerable research. One aspect of this problem is understanding how each muscle in the body contributes to the maintenance of stable positions. The contributions of each muscle are based on its passive elastic and viscous properties as well as its active contraction. Currently there is not a unified method for determining these properties. In this work, methods for determining viscoelastic properties for muscles are explored. Developing a model to predict these values will help build a foundation upon which biomechanical models can be built. The research done in this paper focuses on the biomechanical model of the rat forelimb. Previous work has concentrated on the hind limbs of the rat, and in an effort to fully understand balance control, a biomechanical model of the forelimb is needed.

In order to develop the rat model, architectural data was needed. Rat muscle architecture includes muscle mass, resting muscle length, fiber length, physiological cross-sectional area as well as density, percent slow- and fast-twitch muscle fibers, anatomical cross-sectional area and the architectural index. One of the primary roadblocks to this research was the lack of available rat forelimb data. So, great effort was taken to first complete an extensive literature review for available data. Next when it was discovered rat forelimb data was unavailable, a scaling relationship with 
published forelimb data for another quadruped mammal was needed. In this instance dog forelimb architectural data was used. Rat hindlimb data is available [1] as well as dog hindlimb data [2]. Relationships were established between the dog and rat hindlimb data. Dog forelimb data was discovered from another research paper [3], and the relationships established for the dog - rat hindlimb were used to predict rat forelimb values from the dog forelimb. Many methods were explored in order to scale the data from a dog to a rat.

After the muscle architectural data was determined, the viscoelastic parameters of each muscle needed to be predicted. Currently there does not exist a formal unified approach to determine these values. As a result, a model was developed to predict the spring and damping parameters, which are referred to in this paper as the viscoelastic parameters. Additionally, the active contractile force and the max contractile velocity are determined for each muscle. The approach for determining the muscle parameters in this paper is that of material science, where fundamental muscle properties such as Young's Modulus and geometric data are used to predict values. The linear-Hill muscle model is used to represent each muscle.

After the parameters were set, the model could be developed in the AnimatLab software. The software contains a physics environment where the user can add collision and graphical objects. The files used in AnimatLab were obtained from previous work which studied the rat hindlimb [4]. A few of these models were edited 
to build the forelimb and these mesh models were exported as Standard Triangle Language (STL) files, and input to the AnimatLab physics environment. Once the skeleton was built, the muscles were added. This involved studying three-dimensional anatomical rat models to determine muscle origin and insertion points. These points are then added to the rat skeleton in AnimatLab. The next step is to create muscles between each muscle origin and insertion point. Next, each individual muscle requires the viscoelastic parameters to be set, then the AnimatLab can simulate the model. Finally, the model was compared using visual data from testing a real rats passive muscle properties.

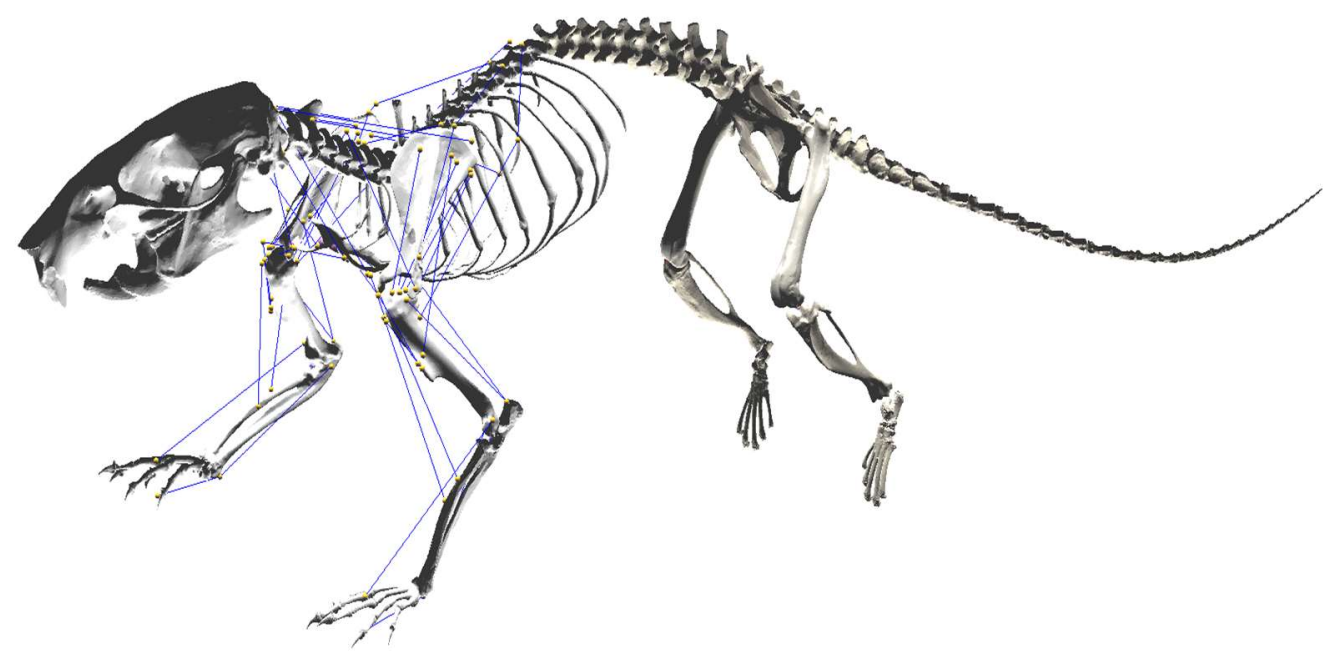

Figure 1.1. The simulated rat in AnimatLab. 


\section{Chapter 2 Background}

\subsection{Biomechanical Models}

The purpose of this work is to develop a biomechanical model of the rat forelimb to a) have a complete model, as a hindlimb model in AnimatLab already exists, and b) to develop a method of predicting viscoelastic muscle parameters. Biomechanical models are used to simulate musculoskeletal systems to recreate and therefore understand motion, forces, momentum, moments, balance, aerodynamics, hydrodynamics, and different forms of locomotion. The benefit of these models is the are able to test models relatively inexpensively when compared to physical robots. Additionally, they provide insight into the behavior of the real biological system they are emulating. Muscles can be stimulated to various degrees of contraction, allowing the user to gain insight into the function of each muscle. Furthermore, different combinations of muscles can be activated providing insight into the overall model behavior. In order to build the biomechanical model of the rat forelimb the skeletal muscle structure must be studied and replicated. The bone segments and joints of the real animal must be replicated. The muscle origin and insertion points must be placed in the best approximate locations. Finally, the viscoelastic parameters must be added to each muscle so it best replicates the real rat. In order to determine the viscoelastic parameters muscle architectural data is needed. While that explains the top-down method of developing the rat biomechanical model, this paper goes from the ground up where the first step is obtaining muscle architectural data, then viscoelastic parameters are established, and finally the model can be built in AnimatLab. 


\subsection{Skeletal Muscle}

Skeletal muscle is the force actuator in animals. Skeletal muscle is what causes animal's limbs to move producing a variety of motion such as running, jumping, swimming, and flying. There are roughly 30 muscles in the rat forelimb alone. The term 'roughly' is used because at times it can be difficult to classify one muscle from many as is the case with the triceps. For example, the triceps contain three distinct muscle bodies, the long, lateral and medial heads, and yet they all converge to share a common insertion point on the olecranon process of the ulna. However, the origin of each of these muscles is distinct. The long head originates in the infraglenoid tubercle of scapula, where the lateral head originates above the radial groove, and the medial head originates below the radial groove. It is the combination of all three of these muscle bodies contracting that allow the elbow to extend. While each muscle only has one-degree of freedom, namely shortening and elongating in the axial direction, it is the placement and combination of the muscle bodies that allows a large degree of freedom for the overall limb. In combination with these muscle bodies, the other types of connective tissue are the tendons which attach the muscle body to bone, and ligaments which hold the bones to one another. However, the muscle body and tendons are very different from each other. While the muscle body is the force generator, the tendon is the unit which enables the force generator to connect to the limb. The tendon has the ability to store a large amount of strain energy, and this energy can be used to reduce muscle fiber work and optimize contraction [5] [6]. 
Exploring the subunits of skeletal muscle reveals smaller and smaller bundles of different components. Skeletal muscle is made up of bundles of fascicles, which in turn are bundles of muscle fibers. Each muscle fiber further breaks down into bundles of fibrils and finally microfibrils. It is the behavior of these muscle myofibrils that determine many of the properties of skeletal muscle.

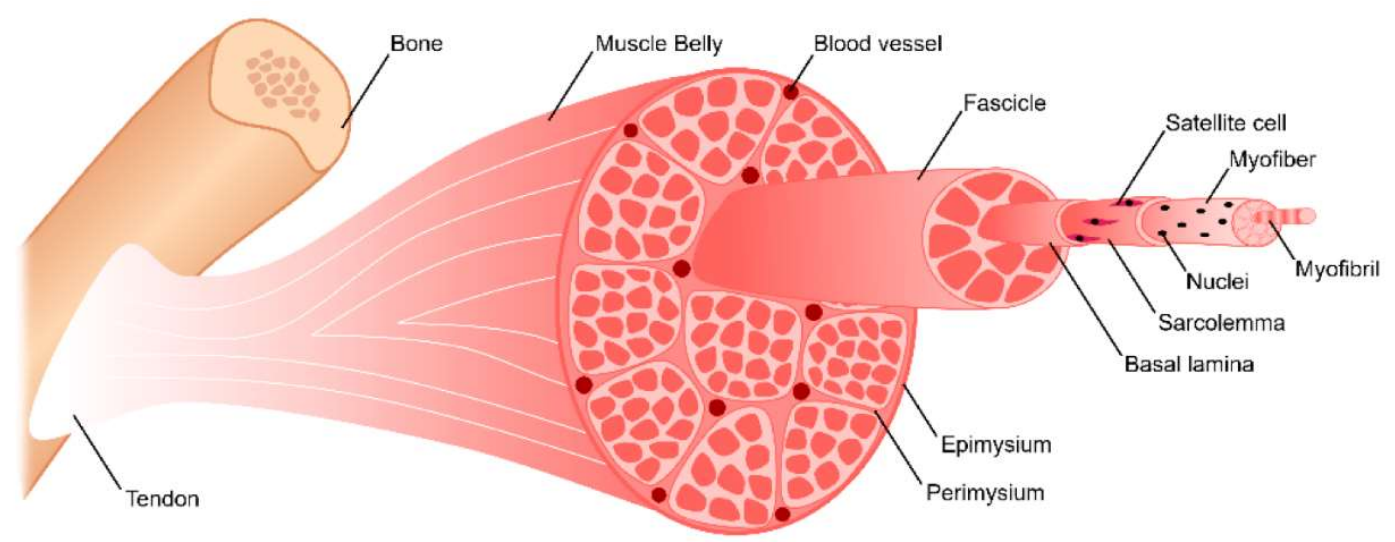

Figure 2.1. The individual components that make up skeletal muscle.

Myofibrils are the basic rod-like unit of the muscle. Myofibrils consist of four complex proteins known as myosin, actin, tropomyosin, and troponin. The proteins are arranged into a functional unit, the sarcomere. 


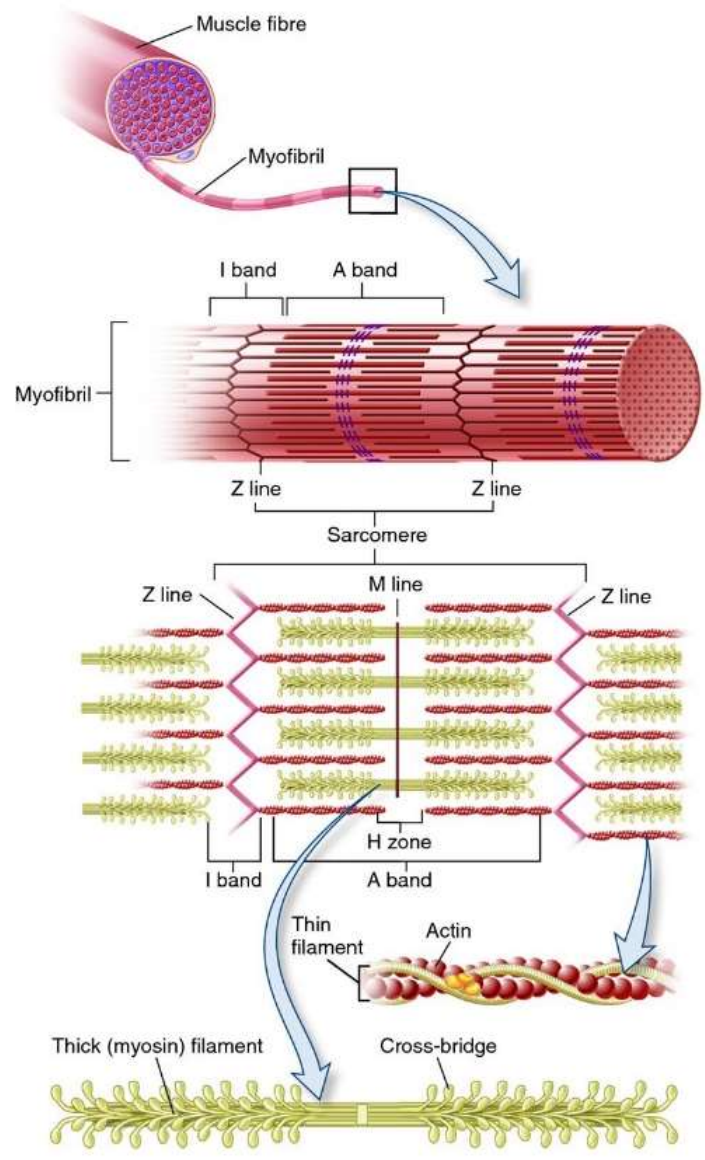

Figure 2.2. Sarcomeres are the underlying mechanisms that generate contractile force.

Muscular contraction is caused by the contraction of the sarcomere. In the muscles resting state, troponin binds tropomyosin to actin preventing myosin from binding to the active sites (myosin - actin binding site). When the nervous system sends a signal down the corticospinal tract via motor neurons the signal is transmitted to the muscle at the neuromuscular junction. This process stimulates skeletal muscle causing an action potential. During the action potential calcium is released from the sarcoplasmic reticulum throughout the muscle. Calcium then binds to troponin which releases tropomyosin from myosin-actin binding sites. Once the active myosin-actin binding 
site is open, the myosin head binds to actin. Energy is provided to the myosin head in the form of Adenosine Triphosphate (ATP) and the myosin head bends causing the sarcomere to shorten. This process is known as the power stroke. The power stroke can continue multiple times causing the sarcomere to shorten even more.

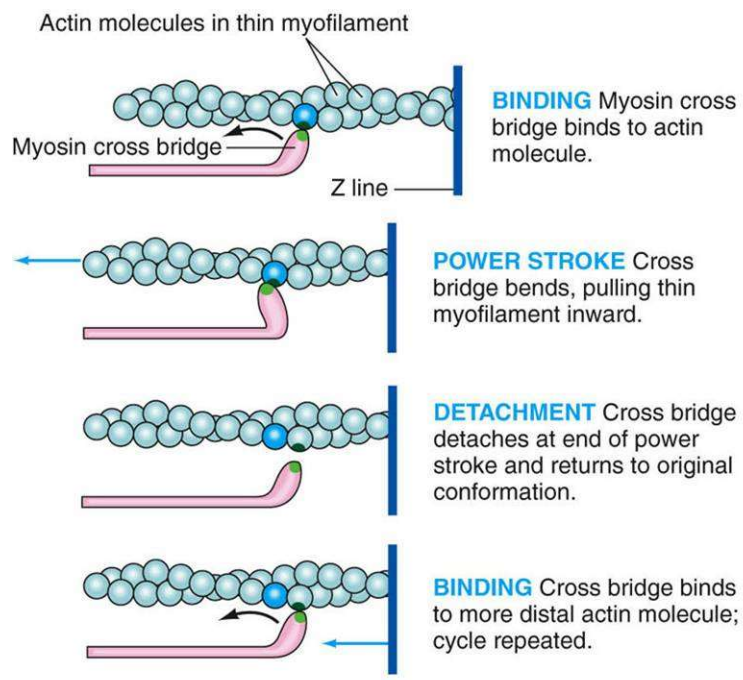

(a)

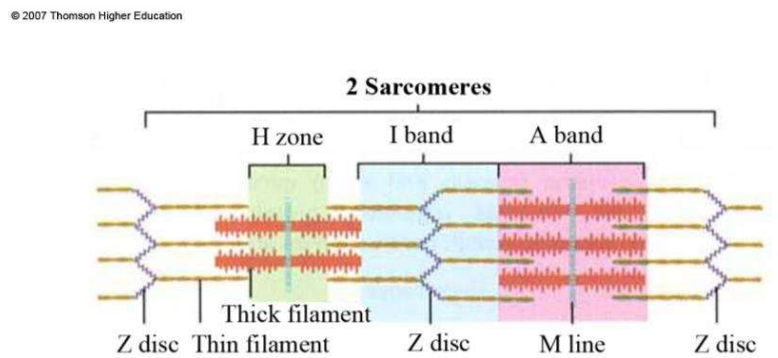

(a) Relaxed musele

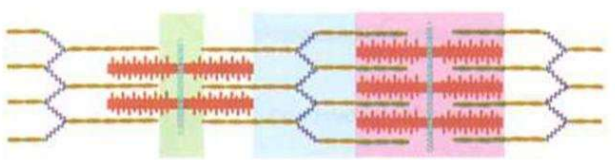

(b) Partially contracted muscle

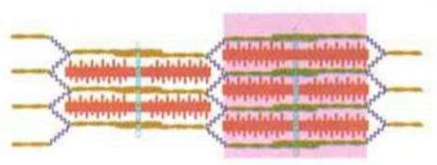

(c) Maximally contracted muscle

Figure 2.3. The power stroke is the interaction of actin, myosin, and ATP. 
Tens of thousands of these sarcomere units are what produce muscle forces [7]. This explanation of muscle contraction was first introduced in 1954 as sliding filament theory [8] [9]. While this is fundamentally how a muscle contracts, it is not the only piece of relevant information. The way in which the muscle attaches to the tendon also determines how the muscle will behave. Furthermore, muscle bodies do not all contain unidirectional muscle fascicles which are all parallel to the muscle body. When the fascicles are all arranged in the same direction they are called fusiform. An example of a fusiform muscle is the biceps brachii. However, a thin portion of the tendon may run through the central region of the muscle body and allow for the fascicles to be arranged at an angle with respect to the tendon, appearing similar to a feather. This arrangement is referred to as bipennate. An example of such an arrangement would be the rectus femoris. Other arrangements are possible such as multipennate (deltoid) and even circular (orbicularus oris). 


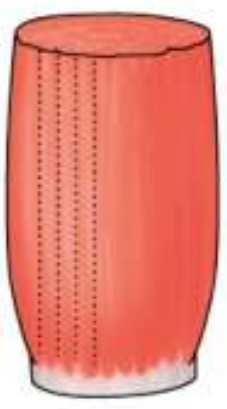

Parallel

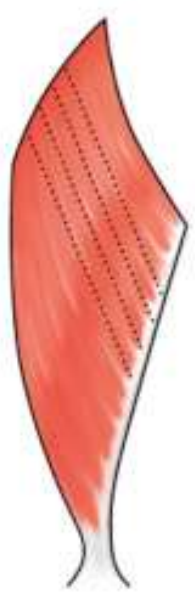

Unipennate

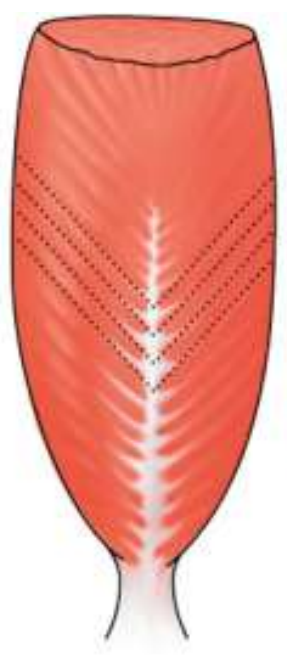

Bipennate

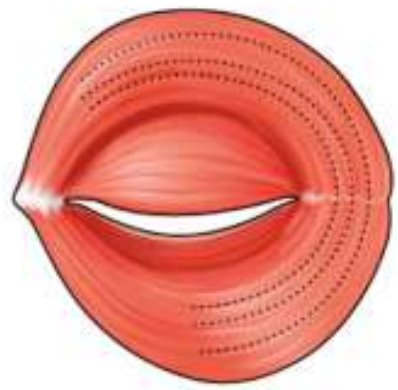

Circular

Figure 2.4. The different types of pennate muscles.

The angle of these fibers with respect to the tendon is called the pennation angle.

When fibers are pennate, more can be packed into a given volume allowing for more force when compared to a parallel-fibered muscle of equal volume. The tradeoff is pennate muscles have shorter fibers, with fewer sarcomeres in series, and therefore their displacements and velocities are lower when compared to parallel muscles with longer fibers [10].

In addition to muscle architectural properties, muscles can also be classified according to their twitch type. Generally, this can be broken into two major categories of slowtwitch or Type I, and fast-twitch, or Type II. Type II muscle fibers can be further subdivided into IIa and IIb. Type IIa muscle fibers are fast-twitch oxidative glycolytic and can be described as fast fatigue-resistant. Type IIb muscles are fast-twitch glycolytic fibers and can be described as fast fatigable. Type I muscles are slow oxidative fibers with high endurance [11]. 
Type I fibers require large mitochondrial volume in order to achieve high endurance. Type II fibers however have a richly developed sarcoplasmic reticulum allowing for more calcium to be deposited on the muscle. High tension requires most of the crosssectional area of fiber to be myofibrils. This is why a muscle cannot be both simultaneously strong, enduring, and rapid [12]. A third type of fast twitch muscle fiber, referred to as Type IIX with myosin heavy chain composition has been identified [11]. However, for the purpose of this research fiber types were only categorized as slow- (Type I) or fast-twitch (Type II). The reason for this was twofold. Firstly, after extensive literature reviews it is very difficult to find the fiber type for each muscle broken down into subcategories of fast-twitch muscle. Secondly, the differences between Type IIa and IIb and how they affect the muscle are not yet described well enough to take into account, other than to say, fast-twitch muscle fibers contract faster and fatigue faster than slow-type muscle fibers. The characteristics of each muscle type are described in table 8 [13].

Table 1. Muscle Fiber Characteristics

\begin{tabular}{llll} 
& $\begin{array}{l}\text { Type I } \\
\text { Slow-Twitch } \\
\text { Oxidative } \\
\text { (SO) }\end{array}$ & $\begin{array}{l}\text { Type IIA } \\
\text { Fast-Twitch } \\
\text { Oxidative } \\
\text { Glycolytic (FOG) }\end{array}$ & $\begin{array}{l}\text { Type IIB } \\
\text { Fast-Twitch } \\
\text { Glycolytic } \\
\text { (FG) }\end{array}$ \\
Characteristics & Slow & Fast & Fast \\
Contraction Speed & Slow & Intermediate & Fast \\
$\begin{array}{l}\text { Fatigue Rate } \\
\text { Diameter }\end{array}$ & Small & Intermediate & Large \\
$\begin{array}{l}\text { ATPase Concentration } \\
\text { Mitochondrial }\end{array}$ & Low & High & High \\
$\begin{array}{l}\text { Concentration } \\
\text { Glycolytic Enzyme } \\
\text { Concentration }\end{array}$ & High & High & Low \\
\hline
\end{tabular}


Skeletal muscle is composed of a heterogenous collection of these muscle fiber types [14] [15]. Additionally, muscle fiber units exhibit plasticity allowing Type IIA and Type IIB to convert between the two as a result of functional training. Type I to Type II conversions are possible in cases of severe deconditioning or spinal cord injury [16]. Another factor contributing to skeletal muscle performance is temperature. As the temperature of the muscle decreases the maximum shortening velocity also decreases [17]. However, temperature effects were not considered in this work and the model is considered to be at a steady state rat body temperature $\left(\sim 37.5^{\circ} \mathrm{C}\right)[18]$.

\subsection{Hill's Mechanical Model of Skeletal Muscle}

Skeletal muscle is the muscle locomotor and even when not actively used it has viscoelastic passive properties [19]. When a limb is moved and the muscle is elongated, the muscle will both resist the change in length as well as dampen changes in length over time. The resistance to the change in length exhibits spring-like behavior comparable to a mechanical spring being stretched with some force. The spring just like the muscle will provide an opposing force until the muscle is relaxed to its resting length. This elastic behavior of skeletal muscle is due to the properties of the muscle body as well as the tendons connecting the muscle body to the bones, often referred to as the muscle-tendon unit. The elastic elements in muscle-tendon unit are able to store energy slowly and release it rapidly, effectively amplifying the muscle power as well as dissipate energy rapidly, such as landing from a jump, to slowly 
stretch the muscle, possibly preventing injury [20]. Many structures within the muscle exhibit these spring-like properties. The elastic properties are due to the actomyosin cross-bridges, actin and myosin filaments, titin, and the connective tissue scaffolding of the extracellular matrix. They all have the potential to store and recover elastic energy during muscle contraction [20]. Tendons, while having a different anatomical structure, also provide a spring-like force [21]. This spring like behavior is due to the collagen that makes up the tendon [22]. The viscous effects demonstrated by the muscle are due to the fluids within the muscle such as extracellular fluid. As the muscle contracts and expands it exhibits a viscous damping-like behavior.

These viscoelastic properties of the muscle are represented in a mechanical model, which is based off the work done in the early to mid-1900s by Hill [23], Huxley [8], and Huxley [9]. The muscle model used in this work is the linear-Hill muscle model shown below. 


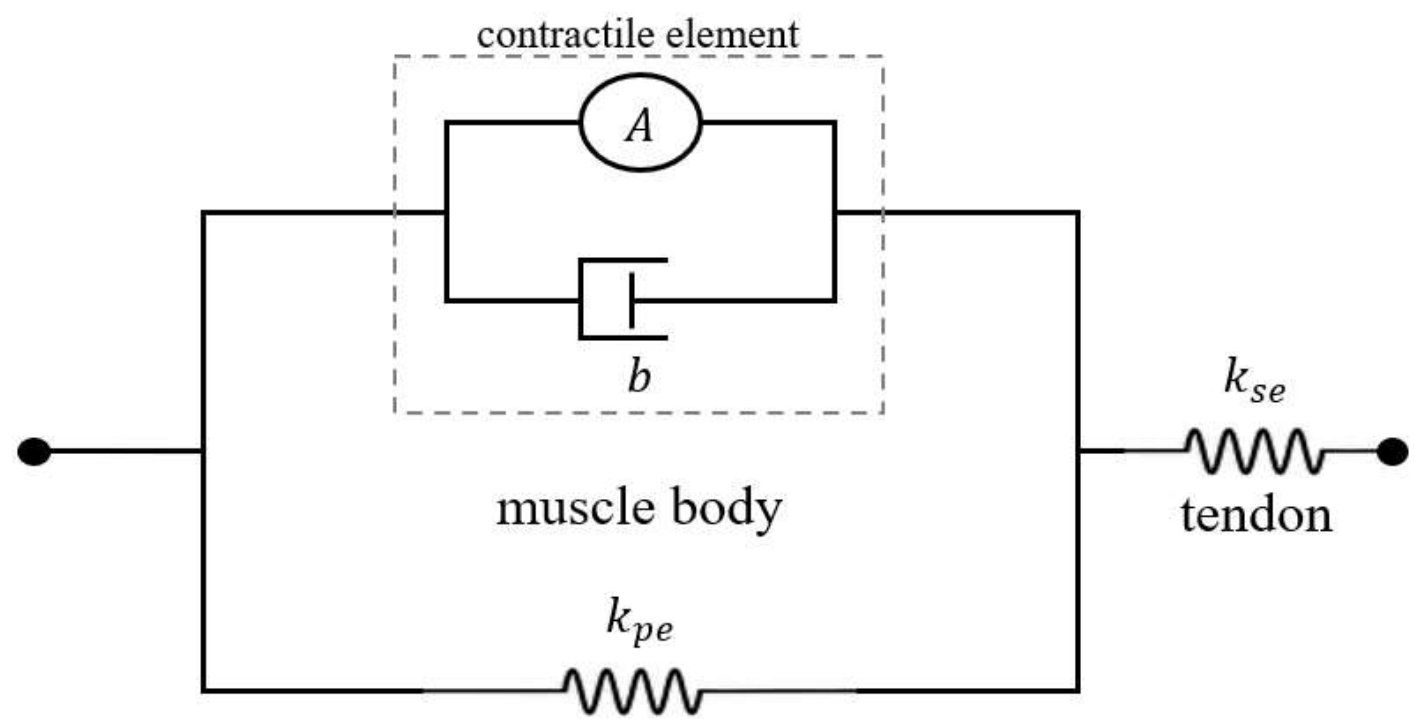

Figure 2.5. The linear-Hill muscle model.

The model consists of a series spring element which can be interpreted as the equivalent spring for both tendons (either side of the muscle body) attaching the skeletal muscle to bone. Additionally, the Hill-muscle model consists of three parallel components which make up the muscle body. The muscle body consists of a spring, damper and active contractile component. The active element is generating the contractile force. Biologically this is produced by the power stroke of myosin and actin interactions within the sarcomere. The governing equation for the Hill-muscle model used in this work is,

$$
\dot{T}=\frac{k_{s e}}{b}\left(k_{p e} \Delta L+b \dot{L}-\left(1+\frac{k_{p e}}{k_{s e}}\right) T+A\right)
$$

where, $\dot{T}$ is the change in muscle contraction force per time, $k_{s e}$ is the series spring element or the tendon/ligament spring constant, $b$ is the damping coefficient for the 
muscle body, $k_{p e}$ is the parallel spring element or the muscle body spring constant, $L$ is the length, $\dot{L}$ is the contractile muscle velocity, $T$ is the measured muscle contraction, and $A$ is the active contractile element. Muscles have only one degree of freedom which is the ability to contract. Due to the elastic spring-like behavior they exhibit, this means they also provide a spring force when stretched beyond their resting length [19]. Therefore, any opposing muscle, such as the triceps (antagonist) to the biceps (agonist) will exert some force opposite to the principal direction. In addition, the antagonist muscle groups will contract to some degree when the agonist is contracting [24]. This is known as coactivation. The degree to which the antagonist muscles activate is not fully described and varies from activity to activity. Coactivation of the antagonist muscle also varies in magnitude with the magnitude of the agonist contraction [24]. 


\section{Chapter 3 Methodology}

\subsection{General outline for methodology:}

The end goal is to develop a biomechanical rat forelimb model. In order to develop the model, viscoelastic parameters for each muscle must be known. In order to determine the muscle parameters, muscle architectural data is needed. Therefore, the methodology for approaching this problem was to first obtain rat forelimb architectural data, then predict passive muscle parameters, and finally build the model. Since rat forelimb architectural data does not exist, a significant amount of work went into predicting values. The approach used in this work is as follows:

Obtain / predict rat forelimb muscle architectural data

- The first step is to obtain architectural data by means of an extensive literature review.

- Once forelimb data was determined to be unavailable, scaling methods from another quadruped mammalian were explored.

- The scaling methods explore different relationships between two quadruped mammalian hindlimbs, namely the rat and dog, and establish relationships between the two in order to predict rat forelimb values.

Predict individual muscle viscoelastic and active parameters

- Determine elastic parameters

- Elasticity of skeletal muscle $\left(k_{p e}\right)$ 
- Elasticity of tendons $\left(k_{s e}\right)$

- Determine the active muscle contractile force $(A)$

- Determine the max contractile velocity $(\dot{L})$

- Determine the viscous effects of the muscle body $(b)$

\section{AnimatLab Model}

- Building the skeleton

- Add muscle origin and insertion points as well as the muscle-tendon unit.

- Setting individual muscle parameters

- $\quad$ Testing the model

\subsection{Determining Rat Forelimb Muscle Architecture}

One of the biggest roadblocks to this research was insufficient architectural data for the rat forelimb. While numerous studies have been conducted on the rat hindlimb [14, $25,1,26,27,28,29]$, in comparison very few have been done on the forelimb. According to an article published by Science magazine in 2021, more than 100 million mice and rats are used in U.S. labs alone [30], yet the literature review did not yield forelimb architecture. When a study involving the forelimb is conducted it is even rarer still to find the muscle geometry of the specimen listed. It is due to this fact, that muscle data had to be estimated from data published on rat hindlimbs as well as those of other quadruped mammals. The general approach was to use data published on rat hindlimbs as well as dog hindlimbs, then to determine a scaling relationship between the two accounting for differences in the proportional length and posture of each 
species. Those relationships were then applied to the forelimb data published for dogs, to determine rat forelimb muscle data. While this is never ideal, others have used a similar approach while achieving satisfactory results [31]. Originally, I attempted to replicate and use this work by using the same scaling relationship reported by Stark. In this work they observed a linear $\log -\log$ (power law) scaling relationship between different dog breeds for both muscle mass and PCSA. The equation for each line was given. Using some of the same dog data that Stark used, which was published by Shahar and Milgram, the same linear relationship was plotted along with the rat hindlimb data.

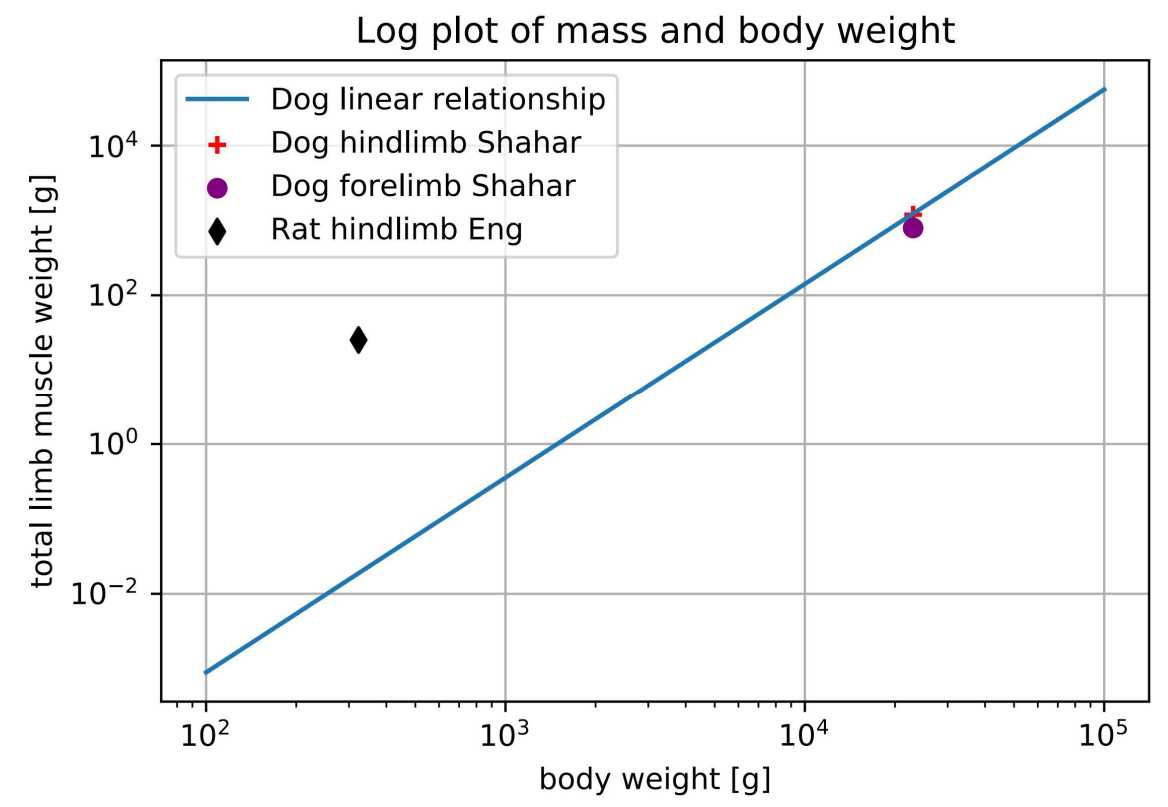

Figure 3.1. Reconstructing the power law relationship between mass and body weight for different dog breeds used by Stark with the addition of the rat.

It became evident the same relationships did not hold for different animals or at least between dogs and rats. 
Although forelimb data was nonexistent, rat hindlimb muscle architecture data was available [1]. This data included muscle mass, resting length, fiber length, pennation angle, PCSA and the architectural index (AI). In order to predict rat forelimb data a relationship needed to be established between dog and rat hindlimbs. Architectural data for dog forelimbs [3] and hindlimbs [2] is published, albeit not from the same dog. Once the data for the rat hindlimb and the dog hindlimb were obtained the next obstacle was matching muscles. The difficulty arose from the fact that different researchers dissect and label muscles differently. For example, one researcher reported muscle values for the cranial and caudal heads of the gracilis and another researcher simply reported the entire muscle as the gracilis. Additionally, even papers published by the same author would label the muscles differently [15] [14]. A direct consequence of this was the limit of comparable data. The rat hindlimb data reported 37 muscles [1], whereas the dog data reported 29 hindlimb muscles [2]. Of these, only 14 muscles of the dog and rat directly overlapped.

\subsubsection{Linear Relationships between the dog and rat muscle architecture}

The first step in investigating the data was to apply a linear relationship between the dog and rat muscle architectures using single linear regression. There were four types of architectural data to compare. These were the muscle mass, muscle resting length, fiber length, and PCSA. Each of the four categories were plotted on four separate scatter plots comparing the dog values to the rat. The dog was the $\mathrm{x}$-axis and the rat 
was the y-axis. Once this was completed using Microsoft Excel, a linear best fit line was added and the $\mathrm{R}^{2}$ values were evaluated.

\subsubsection{Dog Rat Hindlimb Comparison Using a Proportional Length Method.}

A large amount of data has been collected on dog limb proportions. Proportions are defined as the functional length of a bone between joints. Each joint is the start and stopping point of the bone segment regardless if the bone extends beyond the joint. For example, the ulna extends beyond the axis of rotation for the elbow joint. Yet when the ulna is taken into account as some portion of the overall limb, the bone length is considered to end at the elbow joint. In dogs, the radius and ulna account for $30 \%$ of the overall forelimb length on average. The forepaw accounts for $16 \%$. The humerus accounts for $27 \%$ and the scapula accounts for $28 \%$ [32]. The size of each segment various with different sized dog breeds. The mean hindlimb proportions for the dog hindlimb were compared to the hindlimb proportions in the simulated rat model.

Table 2. Proportional lengths of the dog and rat hindlimb.

\begin{tabular}{cccc} 
& Femur proportion [\%] & Tibia proportion [\%] & Hind-paw proportion [\%] \\
\hline Dog & $37 \pm 1.3$ & $37 \pm 1.3$ & $26 \pm 1.5$ \\
\hline Rat & 37.1 & 39.9 & 23 \\
\hline Rat/Dog & 1.00 & 1.08 & 0.88
\end{tabular}


The purpose was to examine the similarities and differences between the different species to see if muscles that scaled with the bone segments could have a scalar applied to them to adjust the muscles from dog to rat hindlimb proportions. For example many of the hindlimb muscles connect from the hip down the entire length of the femur and connect to the bottom of the femur or the top of the tibia. These muscles then scaled with the femur. When a dog is scaled down to the size of a rat, adjustments can be made to account for the different proportional length. Since the femur proportional length was approximately the same as the rat femur proportional length, then no adjusted is needed. However, for muscles whose length scaled with the tibia, the scaled down dog muscles would need to increase by $108 \%$ (Table 1). Similarly, muscles that scaled with the hind-paw would need to be decreased when comparing the scaled down dog with the rat to be $88 \%$ of the dog values. If this model proved to be reliable, the dog and rat forelimb proportions could be evaluated and adjustments could be made to the simple linear scaling of the dog down to the size of a rat.

\subsubsection{Multiple Linear Regression of Separate Clusters Determined by K-Means}

\section{Analysis}

The next method applied was a $k$-means unsupervised machine learning clustering algorithm in the python programming language. The purpose was to determine if there were clusters of muscles similar enough to each other to apply scaling relationships. Each cluster could have its own scaling method instead of trying to scale all the muscles in one cluster. K-means clustering algorithms create as many different 
clusters as are input by the user. Data in a 2-dimensional or 3-dimensional analysis can be plotted allowing the user to observe the data and provide an 'ideal' number of clusters to the model. Data in higher dimensions cannot be visualized and therefore the number of 'ideal' clusters is unknown. In order to determine the number of clusters the "Elbow Method" was used. The Elbow Method takes in the same arguments as the kmeans analysis but outputs how the distortion changes by adding another cluster. Initially the distortion reduces dramatically with more clusters. However, rather quickly, increasing the number of clusters marginally changes the decrease in distortion. Once the slope of these changes goes from a steep drop when adding clusters to a minor change, the "Elbow" can be determined.

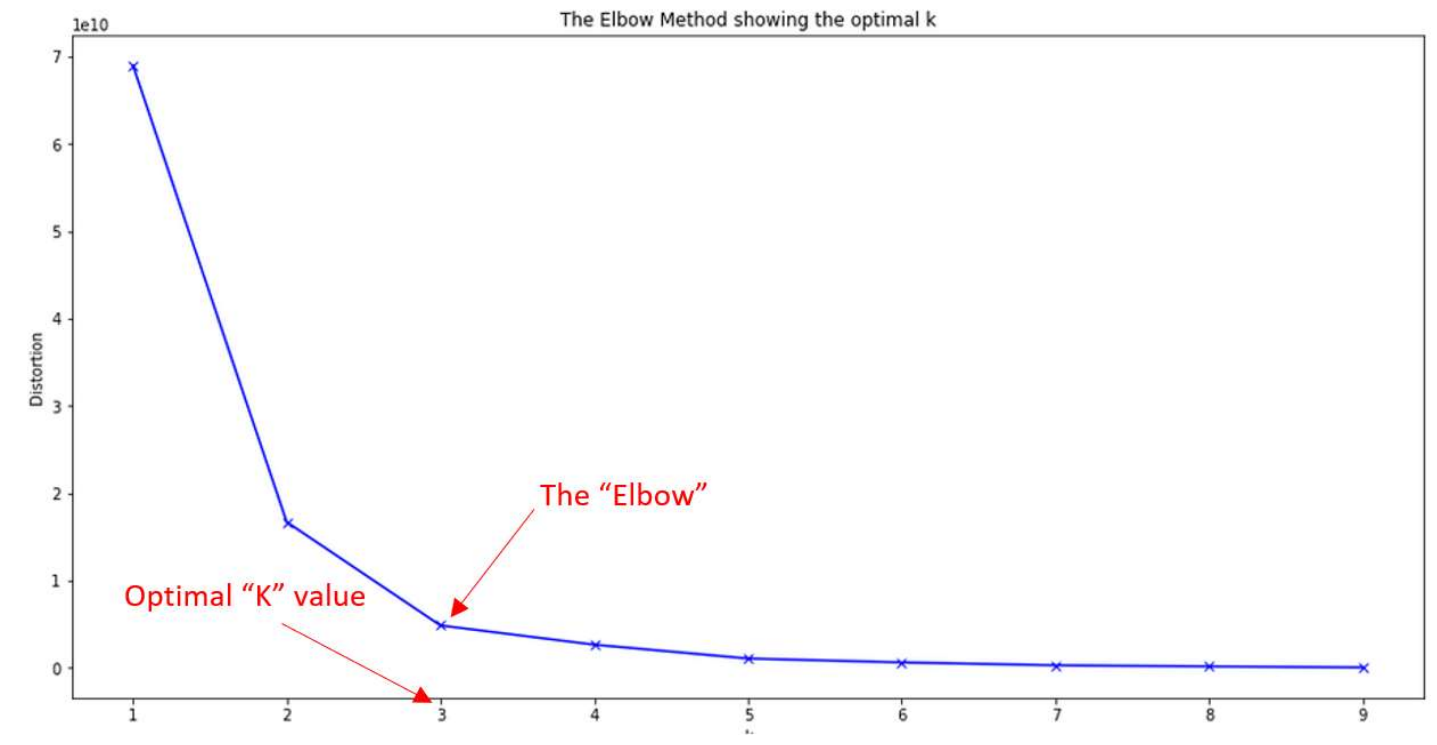

Figure 3.2. The "Elbow Method" shows how the distortion in the system increase as the number of clusters used in k-means analysis increase.

Once the number of preferred clusters are known the k-means algorithm can be used. The purpose was to determine what groups of muscles were similar. If the muscles 
clusters for the dog were similar to the muscle clusters of the rat, then perhaps

different scaling methods could be applied for each cluster. K-means algorithms are able to take higher dimensional data and find relationships between the various data points.

Table 3. The different $k$-means analyses that were used along with their inputs.

\section{Analysis}

Inputs

Dog Only Analysis (DOA) [mass, muscle length, fiber length, PCSA $]_{\operatorname{dog}}$

Rat Only Analysis (ROA) [mass, muscle length, fiber length, PCSA] $]_{\text {rat }}$

Dog-Rat Analysis (DRA) $\quad[\mathrm{m}, \mathrm{ml}, \mathrm{fl}, \mathrm{PCSA}]_{\operatorname{dog}} \&[\mathrm{~m}, \mathrm{ml}, \mathrm{fl}, \mathrm{PCSA}]_{\mathrm{rat}}$

Table 4. The results from a $k$-means analysis. DOA $=$ dog only analysis, $R O A=$ rat only analysis, and $D R A=$ dog-rat analysis.

\begin{tabular}{lccc} 
& \multicolumn{3}{c}{ 3 Cluster Analysis } \\
Muscle Name & DOA & ROA & DRA \\
& Cluster & Cluster & Cluster \\
\hline Gluteus superficialis & 0 & 0 & 0 \\
Adductor longus & 0 & 0 & 0 \\
Extensor digitorum longus & 0 & 0 & 0 \\
Gastrocnemius, medial head & 0 & 0 & 0 \\
Gastrocnemius, lateral head & 0 & 0 & 0 \\
Biceps femoris & 1 & 1 & 1 \\
Semimembranosus & 1 & 1 & 1 \\
Adductor magnus \& brevis & 1 & 1 & 1 \\
Vastus lateralis \& intermedius & 1 & 1 & 1 \\
Gluteus medius & 2 & 2 & 2 \\
Rectus femoris & 2 & 2 & 2 \\
Semitendinosus & 2 & 2 & 2 \\
Gracilis & 2 & 2 & 2 \\
Vastus medialis & 2 & 2 & 2
\end{tabular}


The goal was to see if muscles clustered independently in the rat were similar to muscles clustered independently in the dog. While the analysis did yield there are similarities between the dog muscles and rat muscles, for example the muscles contained in cluster 0,1 and 2 for the dog happen to be the same muscles grouped in clusters 0,1 and 2 for the rat. So, muscles in cluster 0 for the dog have more in common with each other than with muscles grouped in cluster 1 and 2 . The same can be said for the rat. However, the relationships causing the muscles to be grouped together is not clear. For example, the adductor magnus and brevis were in a separate cluster than the adductor longus for both the dog and rat. This data was then compressed to two-dimensions using a principal component analysis (PCA). This enabled a graphical representation of the data.

A multi-linear regression model was then built for each desired prediction (mass, length, fiber, PCSA) for each cluster in python. Each model was trained on an 80/20 split of training/test data. The inputs for the model were always dog muscle mass, length, fiber length and PCSA. The true label for the desired prediction was the rat architecture it was supposed to predict. For example, in order to determine rat muscle mass for a cluster 0 muscle, the model was given the muscle mass, resting muscle length, fiber length and PCSA for each dog muscle in cluster 0 . Then the real rat mass for the same cluster 0 muscle was given to the training set $(80 \%)$ then the model would predict the rat muscle mass for the remaining $(20 \%)$ of the rat cluster 0 muscles. 


\subsubsection{Multiple Linear Regression}

The next method attempted was a multi-linear regression in python without clustering. The number of inputs was varied to see if additional relationships would improve the models. The idea was adding relationships between the data such as density which is a function of mass, muscle resting length and PCSA may improve the models ability to predict any one of those values. The overall methodology was the same as before just without clustering.

Table 5. Coefficients used to determine the rat forelimb data.

\begin{tabular}{lcccccccc}
\multicolumn{1}{c}{$\begin{array}{c}\mathbf{y}= \\
\text { Predicted Rat Value }\end{array}$} & $\mathbf{y}$-intrcpt & mass & length & fiber & PCSA & density & AI & R \\
\cline { 2 - 9 } & $\mathbf{b}_{\mathbf{0}}$ & $\mathbf{b}_{\mathbf{1}}$ & $\mathbf{b}_{\mathbf{2}}$ & $\mathbf{b}_{\mathbf{3}}$ & $\mathbf{b}_{\mathbf{4}}$ & $\mathbf{b}_{\mathbf{5}}$ & $\mathbf{b}_{\mathbf{6}}$ & $\mathbf{b}_{7}$ \\
\hline Mass & 2509.2196 & 0.01 & -212.00 & 189.00 & 27.70 & -3.20 & 0 & 0 \\
Muscle Length & 5.7869888 & 0 & 0.03 & 0.04 & 0.38 & -0.01 & 12.70 & -4.22 \\
Fiber Length & 4.9519382 & 0 & -0.05 & 0.04 & 0.35 & -0.01 & 12.20 & -4.10 \\
PCSA & 0.1922148 & 0 & -0.02 & 0 & 0.03 & 0 & 0 & 0
\end{tabular}

\subsubsection{The Heuristic Method}

The final method used was a heuristic method developed from observation. The dog and rat were observed in a neutral standing position. The idea for this method is to identify differences between the two postures and apply an offset to single linear model. First the single linear relationship was used on dog hindlimb muscle to predict rat hindlimb models. Then the difference, here represented as a quotient, between the 
predicted rat architecture values and real values were determined, using the following equation:

$$
\text { quotient }=\frac{\text { rat }_{\text {real }}}{\text { rat }_{\text {predicted }}}
$$

These values were then plotted.

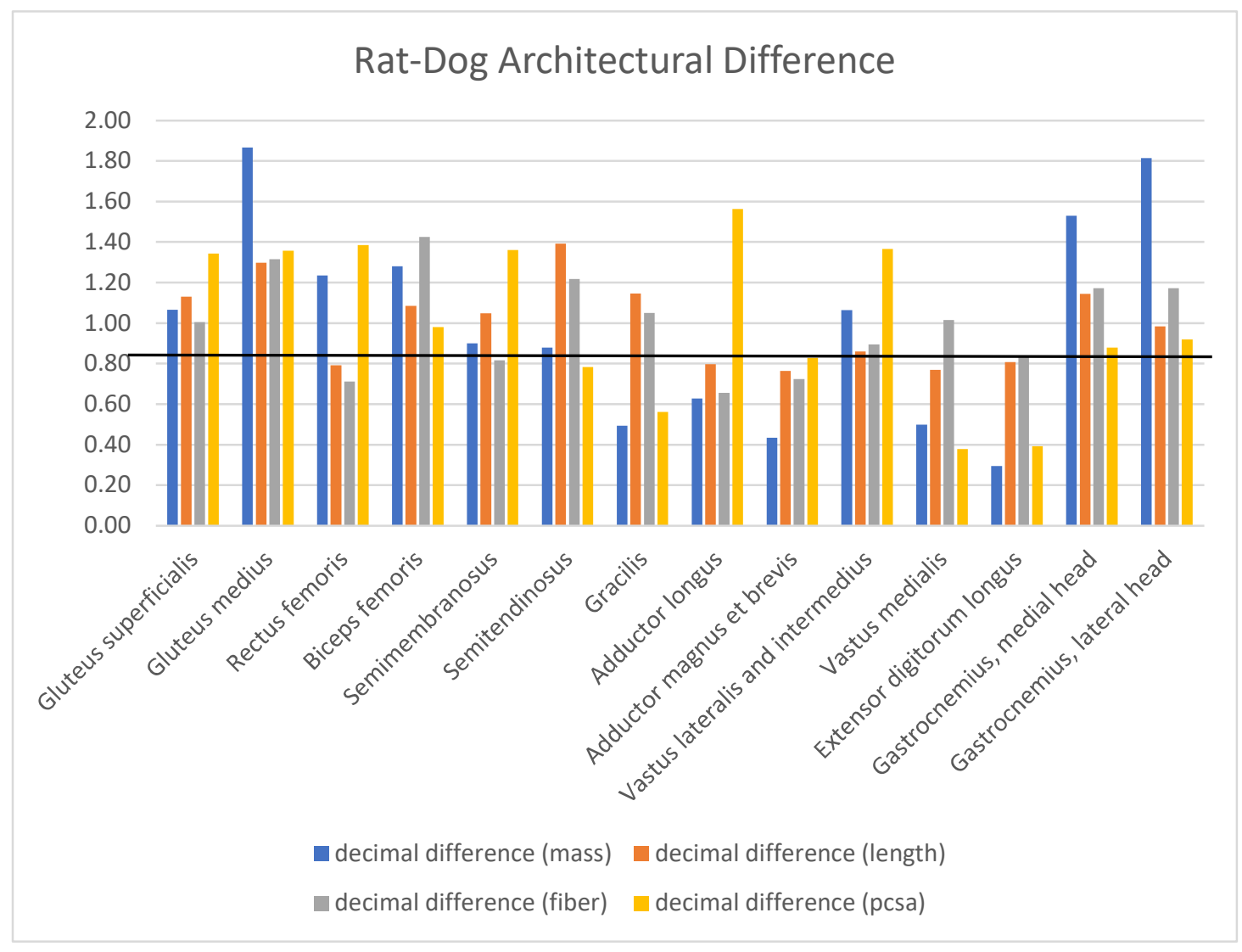

Figure 3.3. The difference between the real and single linear prediction. If the value is 1 the relationship is perfectly linear. 
Next, I had to take into account each muscle's origin and insertion point to see what direction the force was being applied to the forelimb. Based on observational evidence from the rat and dog in standing posture as well as a walking gait, certain aspects stand out. It should be noted Shahar describes the dog used as a 23-kg mixed breed.

Therefore, larger dogs were observed. The first noticeable difference is when a dog is standing the limbs are nearly orthogonal to the ground appearing very upright like a column, whereas a rat has acute angles at the shoulder joint, hip joint, elbow, knee, and ankles.
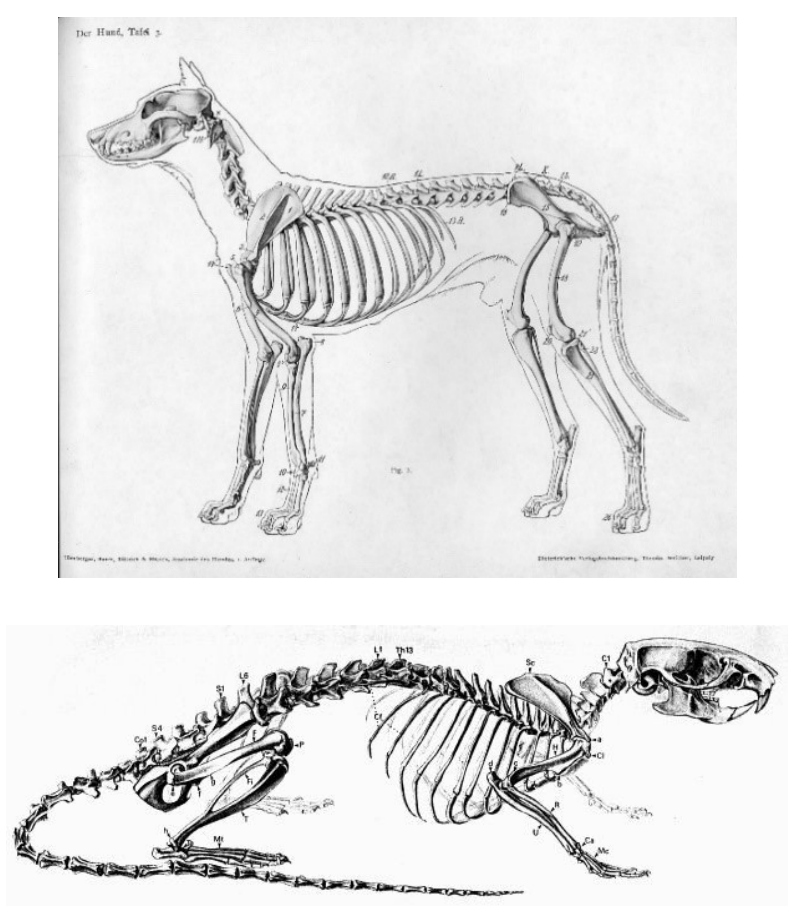

Figure 3.4. The standing posture of a larger dog and a rat.

The reason for this difference in locomotor limb posture is primarily to do with the effect of body mass. A shift in upright posture for larger mammals aligns the segments and joints of the limb with the ground reaction force [33]. Additionally, a shift from a 
crouching more abducted stance to a more upright one decreases the cost (total mechanical power) of locomotion [34]. Because of these observations, the difference between eight groups of muscle functions were chosen to compare the dog and rat hindlimb. Based on the function of each muscle eight classifiers were generated.

Table 6. Muscle classifiers based on function.

\begin{tabular}{cll} 
Class & Hindlimb & Forelimb \\
\hline $\mathbf{1}$ & Adduction & Adduction \\
$\mathbf{2}$ & Abduction & Abduction \\
$\mathbf{3}$ & Hip Flexor & Shoulder Flexor \\
$\mathbf{4}$ & Hip Extensor & Shoulder Extensor \\
$\mathbf{5}$ & Knee Flexor & Elbow Flexor \\
$\mathbf{6}$ & Knee Extensor & Elbow Extensor \\
$\mathbf{7}$ & Dorsiflexor & Dorsiflexor \\
$\mathbf{8}$ & Plantar-flexor & Plantar-flexor
\end{tabular}

Each hindlimb muscle was then assigned a classifier based on its observed function.

Table 7. Hindlimb classifiers.

\begin{tabular}{lcl} 
Muscle Name (Shahar) & Class & Class Name \\
\hline Gracilis & 1 & Adduction \\
Adductor longus & 1 & Adduction \\
Adductor magnus et brevis & 1 & Adduction \\
Biceps femoris & 2 & Abduction \\
Rectus femoris & 3 & Hip Flexor \\
Gluteus superficialis & 4 & Hip Extensor \\
Gluteus medius & 4 & Hip Extensor \\
Semimembranosus & 5 & Knee Flexor \\
Semitendinosus & 5 & Knee Flexor
\end{tabular}




$\begin{array}{lll}\text { Vastus lateralis and intermedius } & 6 & \text { Knee Extensor } \\ \text { Vastus medialis } & 6 & \text { Knee Extensor } \\ \text { Extensor digitorum longus } & 7 & \text { Dorsiflexor } \\ \text { Gastrocnemius, medial head } & 8 & \text { Plantar-flexor } \\ \text { Gastrocnemius, lateral head } & 8 & \text { Plantar-flexor }\end{array}$

At times, a muscle will perform multiple functions. In these instances, like a K-means analysis, the observed primary function was narrowed down to a single class if it exhibited $51 \%$ or more of the overall function. For example, the semitendinosus muscle seems to provide both knee flexion as well as limb adduction. However, based on observation the semitendinosus contributes more to knee flexion than to limb adduction, and therefore it was assigned to the knee flexion class (class-5). Once each hindlimb muscle was assigned to a class, the mean architectural value correction needed was determined for each class in relation to the linearly scaled dog architecture. This provided a structure to use the quasi-linear relationship between the dog and rat while offsetting the muscles based on a difference in muscle function. Since dog and rats have different standing postures, a hypothesis was formed to account for the difference. A muscle used to hold in the rat in a crouch abducted stance will not need to scale directly proportional to the mass of the dog since the dog is in an upright, less abducted posture. Following the same procedure as outlined for the hindlimbs, each forelimb muscle was assigned a class based on its observed function. 
Table 8. Forelimb muscle classes determined by observation.

\begin{tabular}{lcl} 
Muscle Name & Class & Class Name \\
\hline Acromiodeltoid & 4 & Shoulder extensor \\
Acromiotrapezius & 3 & Shoulder flexor \\
Biceps brachii & 5 & Elbow flexor \\
Brachialis & 5 & Elbow flexor \\
Clavotrapezius & - & Clavicle (no class) \\
Cleidomastoid & - & Clavicle (no class) \\
Common digital extensor & 7 & Dorsiflexor \\
Deep digital flexor & 8 & Plantarflexor \\
Deep Pectoral & 1 & Adduction \\
Infraspinatus & 3 & Shoulder flexor \\
latissimus dorsi muscle & 3 & Shoulder flexor \\
Rhomboideus capitis & 4 & Shoulder extensor \\
Rhomboideus Cervicis & 4 & Shoulder extensor \\
Rhomboideus thoracis & 4 & Shoulder extensor \\
Serratus Ventralis & 1 & Adduction \\
Spinodeltoid & 3 & Shoulder flexor \\
Spinotrapezius & 1 & Adduction \\
Subscapularis & 3 & Shoulder flexor \\
Superficial pectoral & 2 & Abduction \\
Supraspinatus & 3 & Shoulder flexor \\
Teres major & 3 & Shoulder flexor \\
Teres minor & 3 & Shoulder flexor \\
Triceps brachii & 6 & Elbow extension \\
\end{tabular}

Once each muscle was assigned a class, the same linear relationship was used. Then the same offsets for each class were applied. In addition to the rat mass, resting muscle length, and PCSA, the muscle fiber type percentage and the tendon data was needed.

\subsubsection{Muscle Fiber Type}

Armstrong found that $95 \%$ of rat hindlimb muscles were composed of fast-twitch muscle fiber [14]. Armstrong also found the fast- and slow-twitch muscle percentage for both the dog fore-and hindlimb. The same heuristic approach from the rat forelimb 
architectural data was used to predict the fiber type percentages for the rat forelimb. A direct comparison between reported dog hindlimb fiber type and rat hindlimb fiber type was used to determine the offset needed for the fast-twitch (Type II) fibers. Once the offsets per class were established, they were applied directly to the dog forelimb fiber type data. Since slow-twitch fibers plus fast-twitch fibers always equal $100 \%$, the model only needed to predict fast-twitch muscle fiber. Slow-twitch fiber percentage was determined by subtracting the fast-twitch percent from $100 \%$.

$$
\text { slow twitch muscle } \%=100-\text { fast twitch muscle } \%
$$

Any muscle that was predicted to have fast-twitch fibers greater than $100 \%$ were then scaled down to be $99 \%$ fast-twitch fiber and $1 \%$ slow-twitch fiber. This was because no muscles in the rat hindlimb were $100 \%$ slow- or fast-twitch.

\subsubsection{Tendon Architectural Data}

While rat hindlimb muscle architecture was difficult to find and rat forelimb architectural data did not exist, rat tendon geometric data was completely absent, except for one brief mention. The mean rat tendon length is reported as 500 microns and 60 microns in diameter [29]. Tendon data, specifically tendon length and tendon cross-sectional area are necessary to predict the elastic behavior. In order to predict the data, I assumed the tendon deviated from the tendon mean in the same way the connected muscle deviated from the muscle mean. For example, if the muscle had a cross-section three standard deviations larger than the mean, then intuitively the 
tendon would also have a cross-section larger than the tendon mean. In order to make the cross-sectional area of the tendon larger, allowing it to connect the larger muscle to bone, the deviation from the tendon mean was assumed to be the same deviation as the muscle it attached to. This enabled me to have different tendon lengths for the different muscles. In order to ensure the deviation of the tendon was the same as the muscle it was connected to, the deviation and mean for each muscle was calculated as well as the population mean and standard deviation. The tendon length was determined using the following function,

$$
L_{t_{i}}=500 \times 10^{-6}[m] \cdot\left(1-\frac{L_{m_{i}}-\mu_{m}}{\mu_{m}}\right)
$$

Where $L_{t_{i}}$ is the tendon length for the $i^{\text {th }}$ muscle. $L_{m_{i}}$ is the resting muscle length for the $i^{\text {th }}$ muscle, and $\mu_{m}$ is the mean for all the muscles. By applying this function to all the tendons, I was able to determine unique values. Similarly, I used the same function but replaced length with diameter to predict tendon diameter values.

$$
d_{t_{i}}=500 \times 10^{-6}[\mathrm{~m}] \cdot\left(1-\frac{d_{m_{i}}-\mu_{m}}{\mu_{m}}\right)
$$

Where $d_{t_{i}}$ is the tendon diameter of the $i^{t h}$ muscle, $d_{m_{i}}$ is the diameter of the $i^{\text {th }}$ muscle, and $\mu_{m}$ is the mean for all the muscles. I was then able to determine the crosssectional area of the tendon using, 


$$
A=\frac{1}{4} \pi d^{2}
$$

With rat forelimb data the next step was to determine the passive parameters for each muscle as well as the active contractile component. I was able to confirm that each tendon deviated from the mean like its corresponding muscle by checking the ratio of individual muscle deviation to population mean.

\subsection{Viscoelastic Passive and Active Parameters}

Currently there is not a straightforward methodology for determining the elastic spring and viscous damping coefficients of linear-Hill skeletal muscle [19]. This work provides a framework/model for determining the viscoelastic parameters as well the active contractile force and max velocity as a function of fast-twitch fiber percentage.

There is some variance in skeletal muscle between different animal species and humans show significant variability between individuals [11]. However, one should expect variance of different muscles across species as well as in the same muscle across the same species. This is because the bicep spring constant of a professional body builder would not be the same as that of an administrator. Additionally, even though they are the same species, one would not expect the max isometric contraction for both individuals to be the same even if they measured the same height, weight, and age. However, if both individuals measured the same height, weight, percent fat, age, as well as individual muscle length and PCSA then one should expect the muscle 
properties would also be nearly identical. Variance among different muscles of the same individual are due to pinnation angle and muscle fiber type [10]. Therefore, an early hypothesis of this research was that fundamentally all mammalian skeletal muscle tissue is the same, and properties should scale with muscle size and shape. This method allows for a material science approach, where given some geometric data, and fundamental properties of the material (crystallin structure for metals, or sarcomere density per unit area for skeletal muscle) predictions can be made about the material. So, given muscle geometry such as PCSA resting length, and percent fastand slow-twitch fiber, muscle properties should be predictable. Physiological crosssectional area is preferred over anatomical cross-sectional area (ACSA), or simply cross-sectional (CSA) area because the PCSA accounts for the pinnation angle $(\alpha)$ of the muscle fibers with respect to the tendon. 

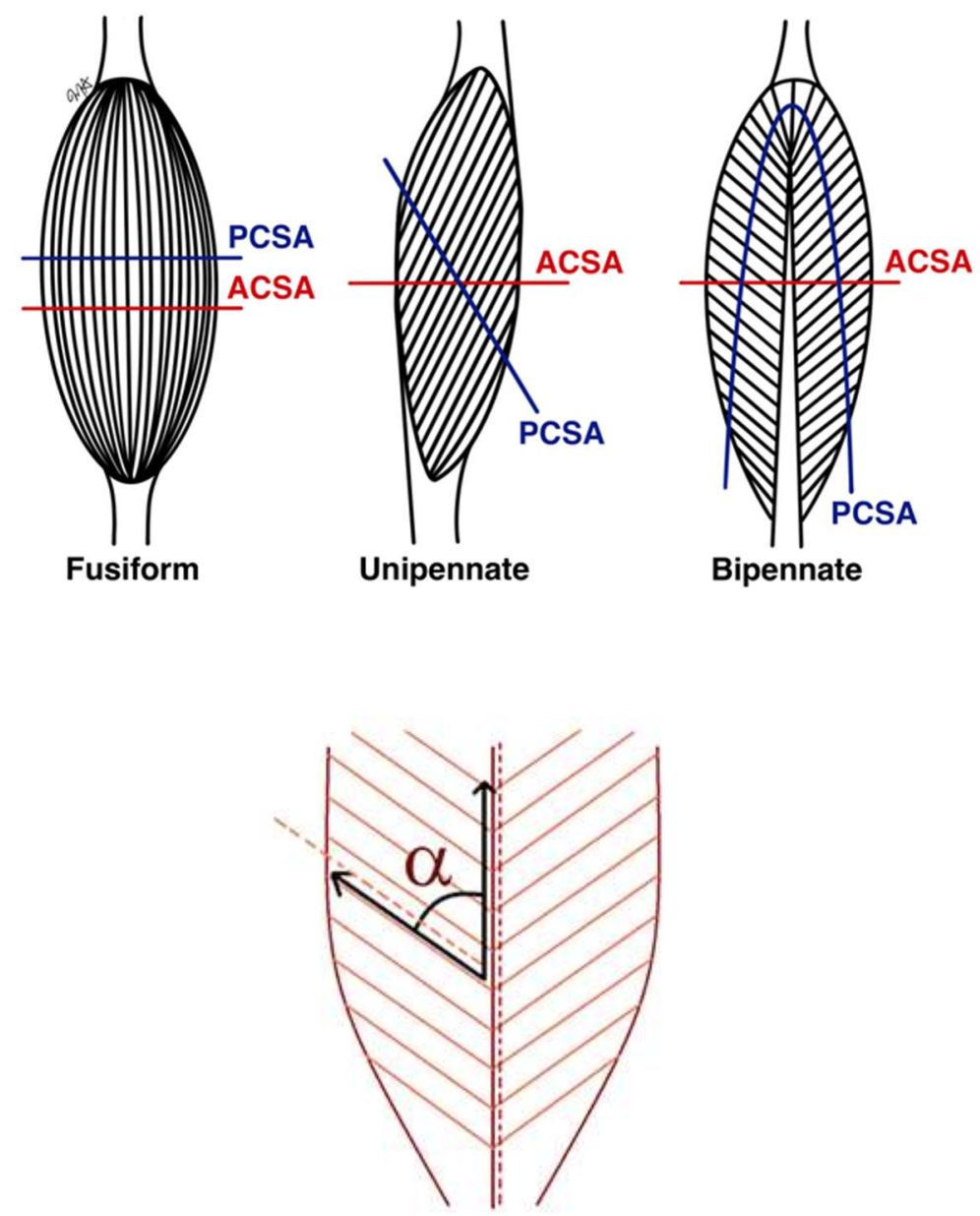

Figure 3.5. The comparison of physiological cross-sectional area (PCSA) and anatomical crosssectional area (ACSA).

An important concept the PCSA captures that the ACSA does not is the number of muscle fibers. When the muscle fibers are not parallel to the tendon or to the direction of desired travel, each sarcomere contraction contributes to the overall change in length as a function of the pinnation angle. Furthermore, when the pinnation angle is not zero, more muscle fibers can be packed into a given volume producing more force when compared to a parallel-fibered muscle of equal volume [10]. This creates for a 
larger muscle activation $(A)$ but as previously described also is inversely proportional to the change in length and therefore contraction velocity.

\subsubsection{Spring Constants}

The spring constants $\left(k_{p e}\right.$ and $\left.k_{s e}\right)$ represent the elastic component of the muscle body and tendon, respectively. In order to determine the elastic spring constant of skeletal muscle, pre-existing relationships of stress and strain were used. The Modulus of Elasticity (or Young's Modulus) for skeletal muscle was reported as $22.59 \pm$ $3.31 \mathrm{kPa}$ for the human medial gastrocnemius, and $23.56 \pm 4.08 \mathrm{kPa}$ for the human lateralis gastrocnemius [35]. As stated earlier, a fundamental hypothesis of this research is all skeletal muscle is the same, therefore the Young's Modulus used for skeletal muscle in this work is $23.08 \mathrm{kPa}$. The stress strain relationship is defined as,

$$
\sigma=E \cdot \varepsilon
$$

Substituting in the values for stress $(\sigma)$ and strain $(\varepsilon)$ yields,

$$
\frac{F}{A}=E \cdot \frac{\Delta L}{L}
$$

This can be put into Hooke's Law form of $F=k \cdot \Delta L$.

$$
F=\frac{A E}{L} \cdot \Delta L
$$


Here the spring constant is,

$$
k_{p e}=\frac{A E}{L}
$$

Where $A$ is the cross-sectional area, $E$ is the modulus of elasticity and $L$ is the resting length. The cross-sectional area used in this work is the PCSA because as indicated previously it takes account the fiber pennation angle and therefore is a more accurate description of the muscle body when you are concerned with elasticity, force, and velocity. Although the elasticity of muscle has non-linear regions [36], an approximation can be made using this linear-Hill model.

The same method and derivation was used for tendons.

$$
k_{s e}=\frac{A E}{L}
$$

Here the differences are: Young's modulus for tendons is reported as $1 \mathrm{GPa}[21]$ and the anatomical cross-section is used because there is no pennation angle of the tendon with respect to itself.

\subsubsection{The Active Contractile Component: Specific Muscle Tension}

As previously discussed, the sarcomere is the fundamental unit providing contractile force of the muscle body. This force has been investigated in relation to area [37] [12]. The resulting force per area is defined as specific muscle tension. Specific muscle 
tension is defined per unit area as $22.5 \mathrm{~N} / \mathrm{cm}^{2}$ [37] [12]. This value can be used to solve for the maximum isometric force $(A)$ through the relationship,

$$
A=22.5 \frac{N}{\mathrm{~cm}^{2}} \cdot \operatorname{PCSA}
$$

A study involving guinea pigs used this specific muscle tension and PCSA to make accurate predictions of the max isometric tetanic tension produced by different individual muscles [37]. Interestingly, a study by Rospars found muscles used for flying and swimming have smaller specific muscle tension than muscles used for terrestrial locomotion [12].

\subsubsection{Viscous Damping Coefficients}

The viscous effects demonstrated by the muscle are due to the fluids within the muscle such as extracellular fluid. As the muscle contracts and expands it exhibits a viscous damping-like behavior. These viscous effects are represented by the damping coefficient $b$. Damping plays a role in how fast the muscle is able to contract as well as the force generated by the muscle. This is best scene in a force-velocity curve for skeletal muscle. 


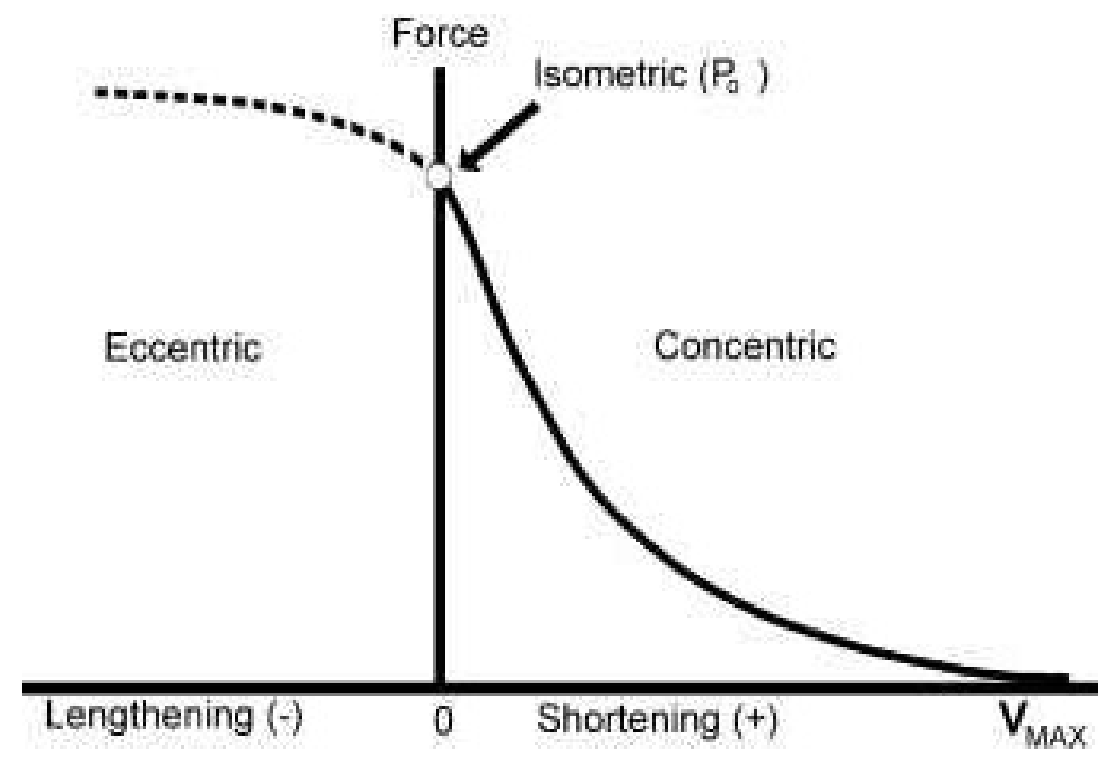

Figure 3.6 Force-Velocity Relationship for skeletal muscle.

It is important to note that any value under the curve is also possible. A muscle can be partially contracted keeping the joint in a fixed location. This specific case would lie on the y-axis as there is no velocity component, however, it would be considerably below the isometric force $y$-intercept. In order to determine the damping effect of skeletal muscle, the governing equation could be arranged to solve for the maximum possible velocity when there is no measured force output. This arrangement puts the solution to the far right of the graph (Figure 3.8). When the change in measured tension and the measured tension are set to zero the governing equation becomes,

$$
b=\frac{-k_{p e} \Delta L-A}{\dot{L}}
$$

$L$ is the resting length and is a given from muscle architectural data. There are two unknowns in this equation. The first being the damping coefficient $(b)$ and the second 
being velocity $(\dot{L})$, in this case maximum velocity. Here the effects of fast- and slowtwitch muscle fiber must be considered. The difference in the maximum velocity produced by both fiber types is [38],

$$
\dot{L}_{\text {max }}=\left\{\begin{array}{l}
\text { fast twitch }=16 l_{0} / \mathrm{s} \\
\text { slow twitch }=6 l_{0} / \mathrm{s}
\end{array}\right.
$$

Where $l_{0}$ is the resting muscle length and $s$ is seconds. This equation can be rewritten in a single function to allow a continuum of values.

$$
\dot{L}_{\text {max }}=\left(6+\left(10 \cdot N_{f t}\right)\right) * l_{0} / s
$$

Where $N_{f t}$ is the number of fast-twitch muscle fibers. Now if the number of fasttwitch muscle fibers is zero the equation becomes the same as 3.13 for slow-twitch muscle fibers and if the number of fast-twitch muscle fibers is $100 \%$ then the equation becomes the same as 3.13 for fast-twitch muscle fibers. All values are now known and $b$ can be solved for. It should also be noted that the Hill muscle model treats damping as linear. Therefore, it creates a straight line between the max force and the max velocity. This is a limitation of the model that would therefore overestimate the amount of force that can be produced at a specific velocity for nearly all cases except the max velocity and max force. 


\subsubsection{The Muscle Parameter Model}

The purpose of developing these relationships was to establish the muscle parameter model. The reason for creating this model, which is shown collectively below, was due to the fact there is not a single established methodology for predicting muscle parameters. The advantage of this approach is to use a first-principles material science approach whereby understanding the fundamental mechanisms at work such as the sarcomere and myofibril as well as their interactions with other fundamental mechanisms such as the tendon and muscle body allows for a predictive model to be built using only the muscle geometry (PCSA and resting length) as well as the major fiber type (percentage fast-twitch). This can be summarized in the following equations:

The elastic spring force due to the muscle body (Equation 3.9),

$$
k_{p e}=\frac{E_{m u s c l e} \cdot P C S A}{L}
$$

The elastic spring force due to the tendons (Equation 3.10),

$$
k_{s e}=\frac{E_{\text {tendon }} \cdot P C S A}{L}
$$

The max active contractile component (Equation 3.11),

$$
A_{\max }=22.5 \frac{\mathrm{N}}{\mathrm{cm}^{2}} \cdot P C S A
$$


The viscous damping effect due to the muscle body (Equation 3.12),

$$
b=\frac{-k_{p e} \Delta L-A}{\dot{L}}
$$

Where (Equation 3.14),

$$
\dot{L}_{\max }=\left(6+\left(10 \cdot N_{f t}\right)\right) * l_{0} / s
$$

\subsection{Building the model in Animatlab}

The first step in designing a simulated biomechanical model of a rat forelimb was to determine how the forelimb operated and how the skeleton moved through space. Through observation of a rat in motion I was able to determine the forelimb model uses two ball-and-socket joints to model clavicle and scapula movement. A third balland-socket joint is used at the shoulder and two hinge joints are used at the elbow and wrist. Scapula motion is further constrained by muscle and spring elements. Each forelimb has 11 degrees of freedom, and 23 Hill-type muscles. 

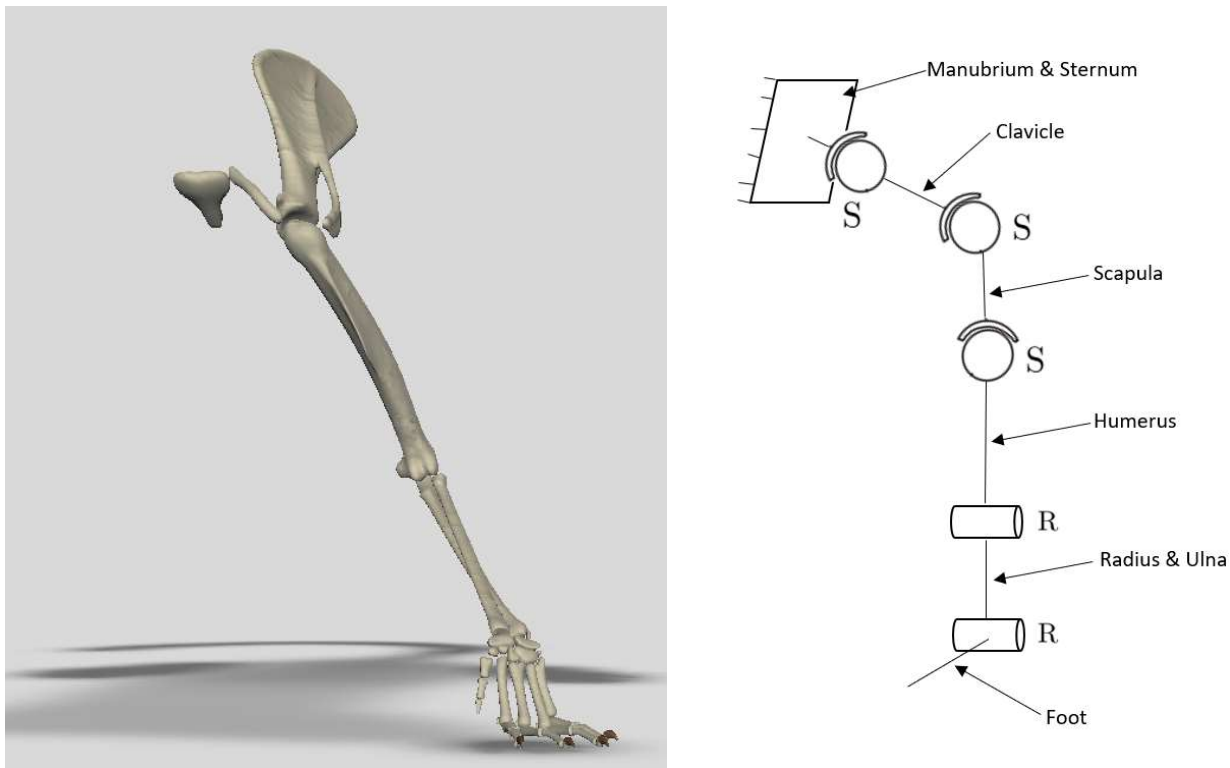

Figure 3.7. The rat forelimb is comprised of three spherical joints and two revolute joints.

This model can be further described mathematically by establishing the location and orientation of each limb. 


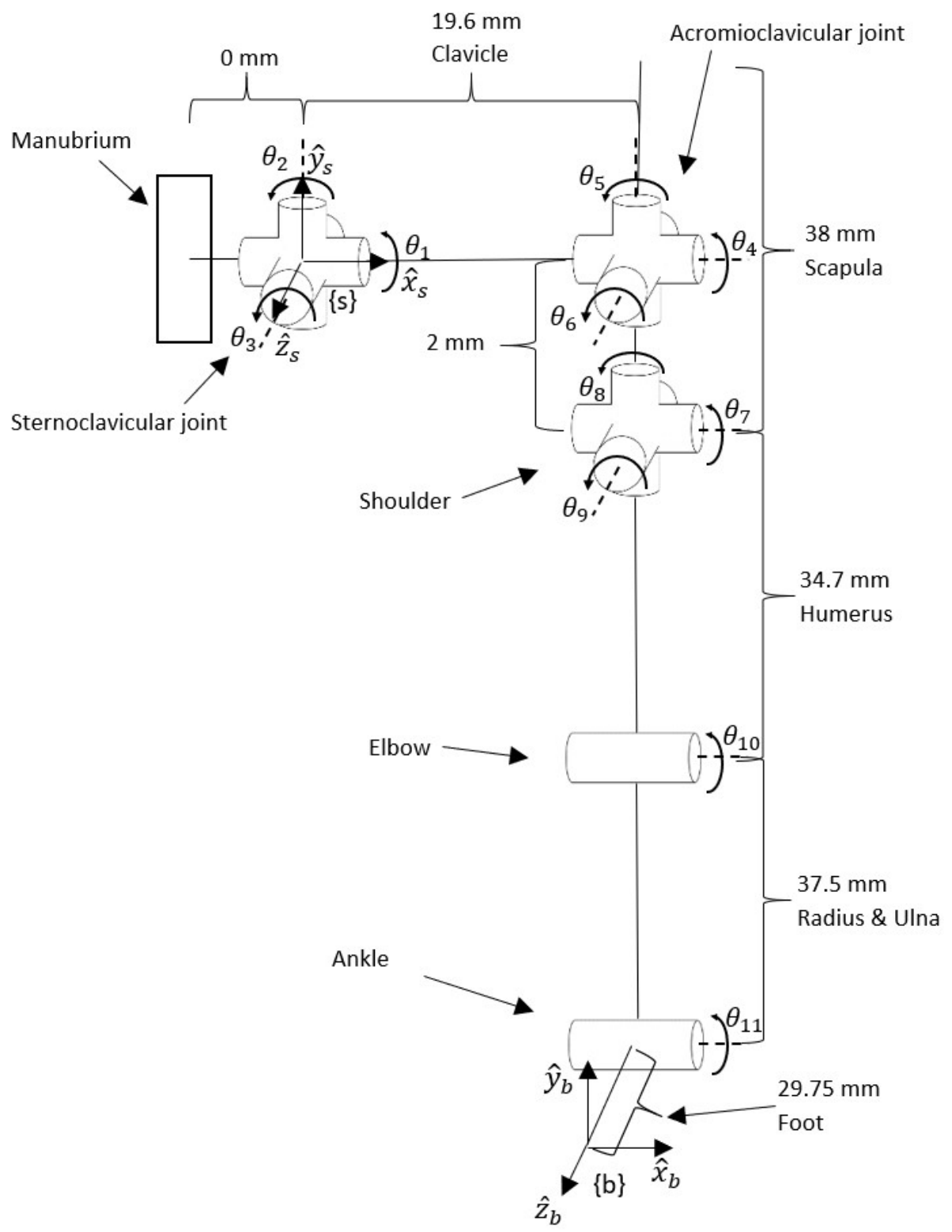

Figure 3.8. The mathematical system describing the movement of the rat forelimb.

After determining the degrees-of-freedom the next step was to determine the muscles being used and how they are connected to the skeleton. A software called Biosphera3d 
was used to get a 3D visualization of the rat and determine the muscle to bone origin and insertion points. Each muscle attaching to the forelimb, including the clavicle, scapula, humerus, radius, ulna and smaller bones of the wrist and hand were documented, and the attachment points were recorded. The attachment points were then added to the AnimatLab model.

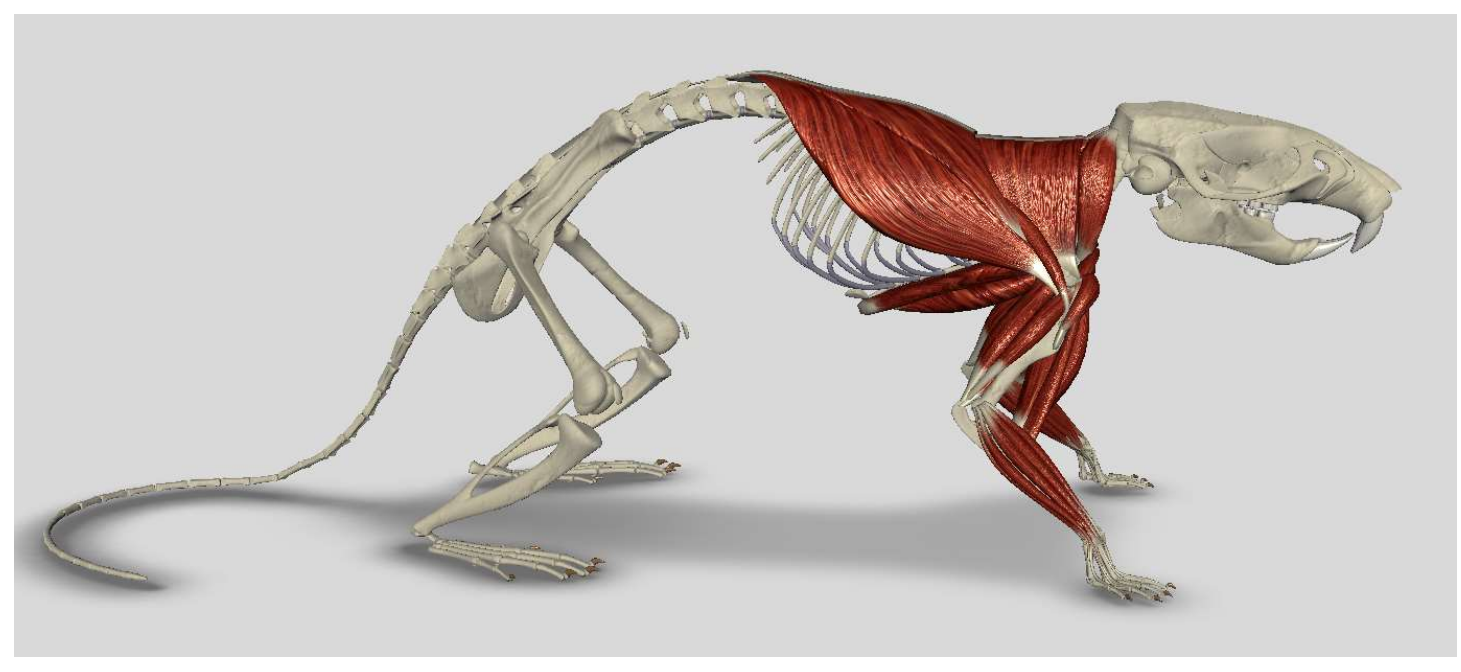

Figure 3.9. The muscles used to manipulate the forelimbs of the rat. Image taken from the Biosphera3d software.

The next step in the process was to determine the muscle path. Each muscle group (such as the biceps) would be represented by a single muscle in the simulated model. This proved to be very challenging as many of the muscles included multiple attachment points, such as the acromiodeltoid muscle which connects the scapula to the clavicle as well as the humerus, or the triceps brachii muscle which attaches the scapula to the humerus and the ulna. Further complications arose when muscles 
attached to the spine or rib cage such as acromiotrapezius muscle which attaches the scapula to multiple segments of the cervical and thoracic spine. Here the center point of the attachment surface was approximated and added to the AnimatLab model. Once the model was built the parameters $k_{s e}, k_{p e}, b$, and $A$ were added to the model for each muscle. In order to test the model, it needed to be compared to a video of a real anesthetized rat whose limbs were lifted and dropped to examine how the passive muscle parameters behaved. The last step was to manipulate the simulated AnimatLab model's limbs to be in the same orientation as the real rat model and then run the simulation to compare the behavior. The model was compared side-by-side with the real rat for two different tests and at different speeds. The first test tested passive muscle properties as the rat forelimb was extended posterior of the shoulder and released. The second test placed the rat forelimb anterior of the shoulder and released. The simulated model's forelimb was placed in the same approximate location for test 1 and test 2 and the video results were compared side by side. The video is played at $1 / 8^{\text {th }}$ speed, for test 1 and 2 , then $1 / 4$ speed for test 1 and 2 , and finally both tests are played in real time. The purpose was to allow the viewer time to fully examine the models, as the tests took less than one second. 


\section{Chapter 4 Results}

\subsection{Muscle Architecture Predictions}

Here are the results from each of the models used to predict rat muscle architecture, given rat and dog muscle architectural data.

\subsubsection{Linear relationships between the dog and rat muscle architecture.}
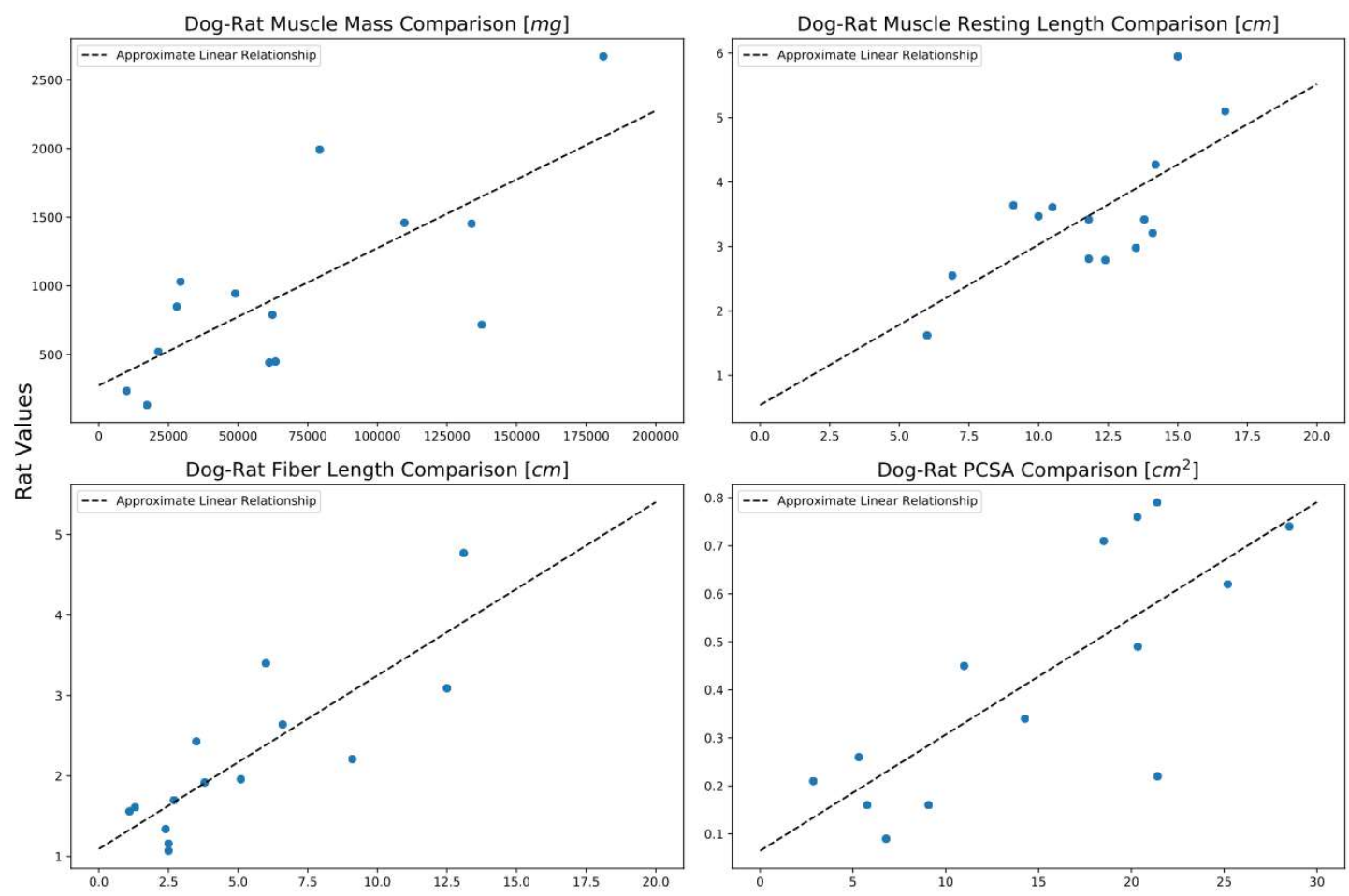

Figure 4.1. Dog-Rat muscle architecture approximate linear relationships.

\subsubsection{Dog Rat Hindlimb Comparison Using Proportional Lengths}

The predicted rat hindlimb values were compared to the real rat hindlimb values and the percent error for each of the four categories (muscle mass, muscle resting length, fiber length, and PCSA) were calculated. 
Table 9. The mean percent error for each predicted value when compared to the real value.

\begin{tabular}{lcccc} 
Architecture & Muscle Mass & Muscle Resting Length & Fiber Length & PCSA \\
\hline \% error \pm std & $62.83 \pm 93.50$ & $10.30 \pm 23.36$ & $7.98 \pm 25.29$ & $35.64 \pm 71.24$
\end{tabular}

\subsubsection{Multiple Linear Regression of Separate Clusters Determined by K-Means}

\section{Analysis}

The "Elbow Method", which was completed in Python, suggested three clusters.

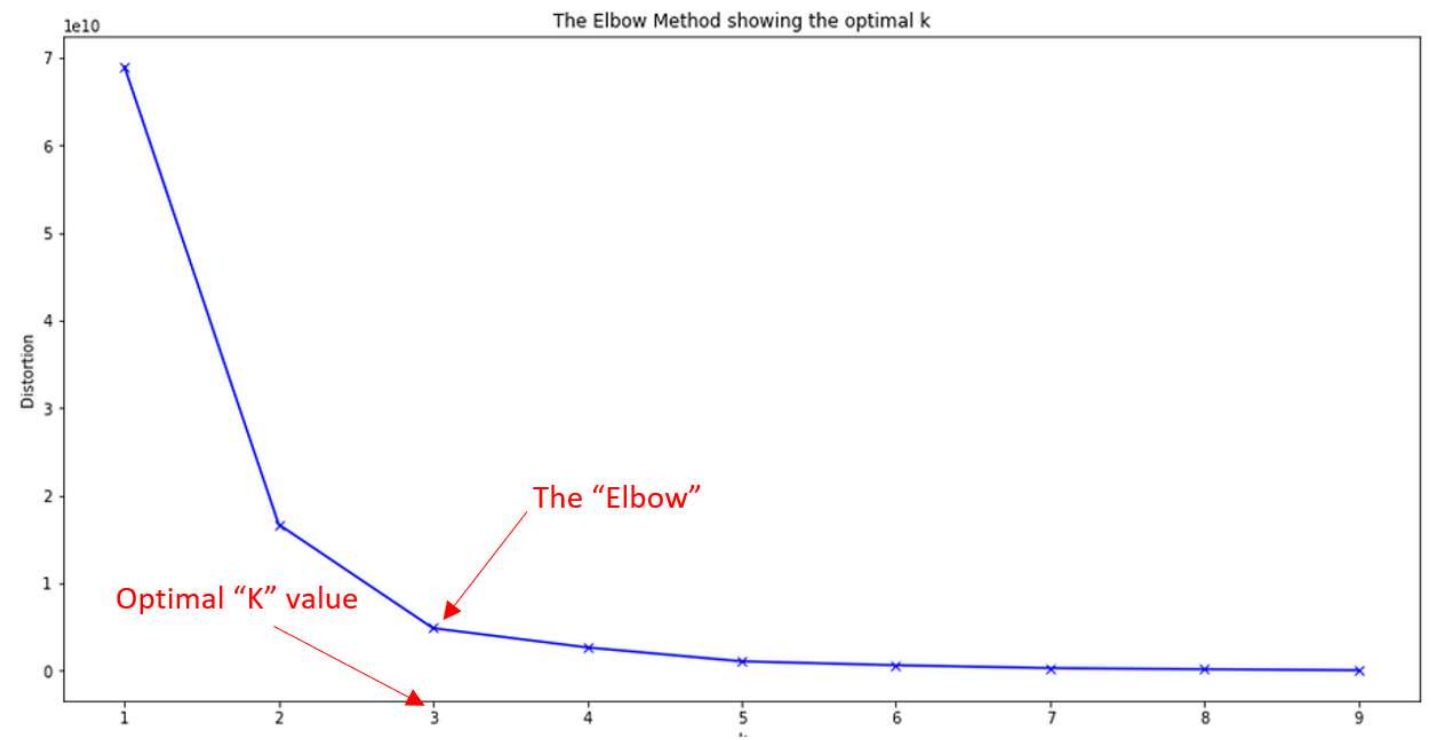

Figure 4.2. The results from the "Elbow Method" used in python to determine the number of clusters needed for analysis.

As a result, a three cluster study was conducted. 12 multiple linear regression models were used and their $R^{2}$ values were compared. 
Table 10. The k-means clustered multi-linear regression models used to predict rat muscle architecture. Each row is a different model.

\begin{tabular}{clll} 
Cluster & \multicolumn{1}{c}{ Model Inputs (dog data only) } & Predicted Rat Value & $\mathbf{R}^{\mathbf{2}}$ \\
\hline 0 & mass, resting length, fiber length, PCSA, density & Mass & 0.97 \\
0 & mass, resting length, fiber length, PCSA, density & Resting Length & 0.89 \\
0 & mass, resting length, fiber length, PCSA, density & Fiber Length & 0.91 \\
0 & mass, resting length, fiber length, PCSA, density & PCSA & 0.95 \\
1 & mass, resting length, fiber length, PCSA, density & Mass & 0.56 \\
1 & mass, resting length, fiber length, PCSA, density & Resting Length & 0.37 \\
1 & mass, resting length, fiber length, PCSA, density & Fiber Length & 0.6 \\
1 & mass, resting length, fiber length, PCSA, density & PCSA & 0.69 \\
2 & mass, resting length, fiber length, PCSA, density & Mass & -3 \\
2 & mass, resting length, fiber length, PCSA, density & Resting Length & -1.2 \\
2 & mass, resting length, fiber length, PCSA, density & Fiber Length & 0.54 \\
2 & mass, resting length, fiber length, PCSA, density & PCSA & -2.8
\end{tabular}

\subsubsection{Multiple Linear Regression}

Multiple linear regression was used to predict the four desired architectural values.

The number of inputs was increased in an effort to increase the models accuracy. The model started with just the four architectural values (mass, resting length, fiber length, and PCSA). However, relationships between these values such as density which is calculated used mass, resting length and PCSA, or the architectural index (AI) which is defined as the quotient of fiber length to muscle resting length, and finally the radius $(R)$ which was approximated as $\sqrt{P C S A / \pi}$ were added as inputs to improve the models accuracy. 
Table 11. The multi-linear regression models trained on dog and rat hindlimb data used to predict forelimb data.

\begin{tabular}{llc}
\multicolumn{1}{c}{ Model Inputs (dog data only) } & \multicolumn{1}{c}{ Predicted Rat Value } & $\mathbf{R}^{2}$ \\
\hline mass, resting length, fiber length, PCSA & Mass & 0.73 \\
mass, resting length, fiber length, PCSA & Resting Length & 0.73 \\
mass, resting length, fiber length, PCSA & Fiber Length & 0.73 \\
mass, resting length, fiber length, PCSA & PCSA & 0.61 \\
mass, resting length, fiber length, PCSA, density & Mass & 0.76 \\
mass, resting length, fiber length, PCSA, density & Resting Length & 0.77 \\
mass, resting length, fiber length, PCSA, density & Fiber Length & 0.75 \\
mass, resting length, fiber length, PCSA, density & PCSA & 0.61 \\
mass, resting length, fiber length, PCSA, density, AI & Mass & 0.77 \\
mass, resting length, fiber length, PCSA, density, AI & Resting Length & 0.82 \\
mass, resting length, fiber length, PCSA, density, AI & Fiber Length & 0.82 \\
mass, resting length, fiber length, PCSA, density, AI & PCSA & 0.61 \\
mass, resting length, fiber length, PCSA, density, AI, R & Mass & 0.79 \\
mass, resting length, fiber length, PCSA, density, AI, R & Resting Length & 0.90 \\
mass, resting length, fiber length, PCSA, density, AI, R & Fiber Length & 0.94 \\
mass, resting length, fiber length, PCSA, density, AI, R & PCSA & 0.59
\end{tabular}

Using the best $R^{2}$ value for each of the predicted values, the models were then used to predict forelimb values. 


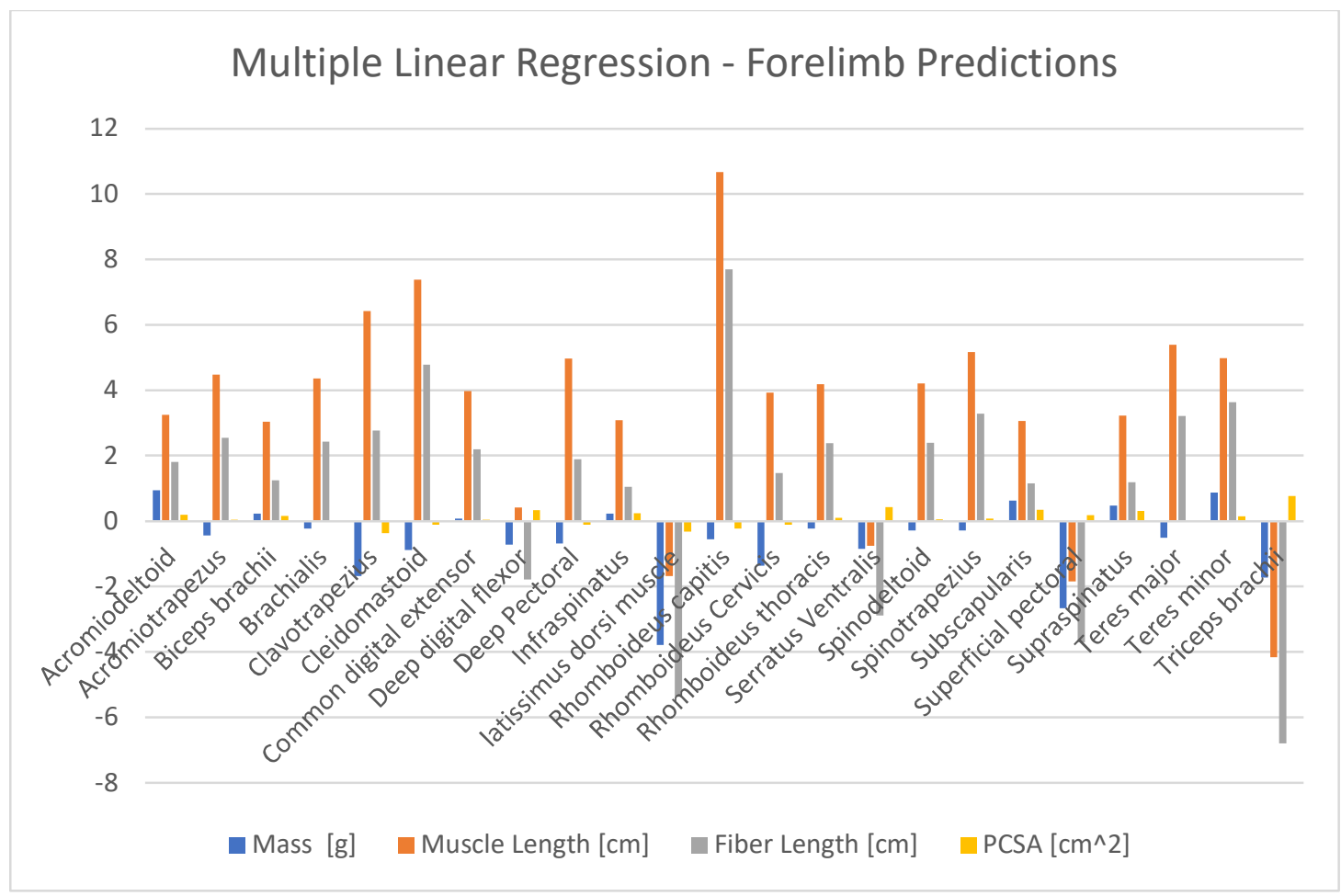

Figure 4.3. The predicted forelimb values using the multiple linear regression models.

\subsubsection{The Heuristic Method}

The first step of the heuristic method was to apply the single linear regression model.

Table 12. Slope intercept equations used.

\begin{tabular}{lccc}
\multicolumn{1}{c}{ Rat Predicted Value } & Slope & y-intercept & $\mathbf{R}^{\mathbf{2}}$ \\
\hline Muscle mass & 0.01 & 275.18 & 0.54 \\
Resting muscle length & 0.2491 & 0.5384 & 0.51 \\
Fiber length & 0.2155 & 1.0931 & 0.69 \\
PCSA & 0.0242 & 0.0649 & 0.61
\end{tabular}

The quotient of real to predicted hindlimb values were plotted. If the quotient equals one then the relationship is perfectly linear, otherwise an offset was needed. 


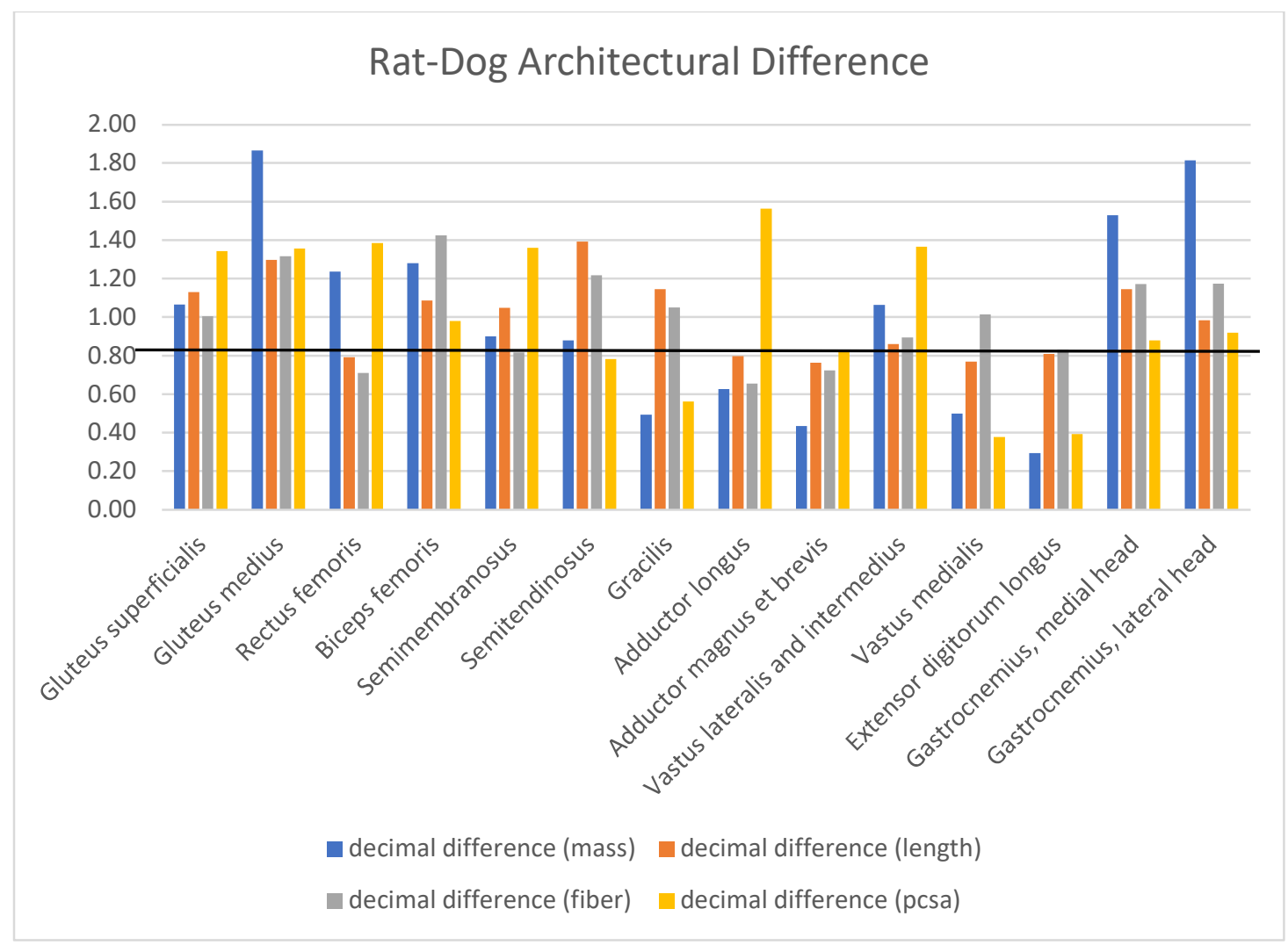

Figure 4.4. The difference between the real and single linear prediction. If the value is 1 the relationship is perfectly linear. 
Once each muscle was assigned to a class, the offset needed to make the quotient of real to predicted rat data equal to one, was calculated.

Table 13. Shift necessary for each difference between the linear rat-dog model and the heuristic approach. Values are given in mean \pm standard deviation.

\begin{tabular}{clcccc} 
Class & Class Name & mass & length & fiber & PCSA \\
\hline 1 & Adduction & $0.52 \pm 0.08$ & $0.90 \pm 0.17$ & $0.81 \pm 0.17$ & $0.98 \pm 0.42$ \\
2 & Abduction & $1.28 \pm 0$ & $1.09 \pm 0$ & $1.42 \pm 0$ & $0.98 \pm 0$ \\
3 & Shoulder flexors & $1.24 \pm 0$ & $0.79 \pm 0$ & $0.71 \pm 0$ & $1.38 \pm 0$ \\
4 & Shoulder extensors & $1.47 \pm 0.4$ & $1.21 \pm 0.08$ & $1.16 \pm 0.16$ & $1.35 \pm 0.01$ \\
5 & Elbow flexors & $0.89 \pm 0.01$ & $1.22 \pm 0.17$ & $1.02 \pm 0.2$ & $1.07 \pm 0.29$ \\
6 & Elbow extensors & $0.93 \pm 0.29$ & $0.81 \pm 0.05$ & $0.87 \pm 0.06$ & $1.04 \pm 0.5$ \\
7 & Dorsiflexion & $0.29 \pm 0$ & $0.81 \pm 0$ & $0.83 \pm 0$ & $0.39 \pm 0$ \\
8 & Plantarflexion & $1.67 \pm 0.14$ & $1.06 \pm 0.08$ & $1.17 \pm 0.00$ & $0.90 \pm 0.02$
\end{tabular}

The predicted heuristic values were a combination of the single linear regression values with the addition of the classifier offset (Table 13). These predicted heuristic values were then compared to the single linear regression model as well as the real rat hindlimb data. 


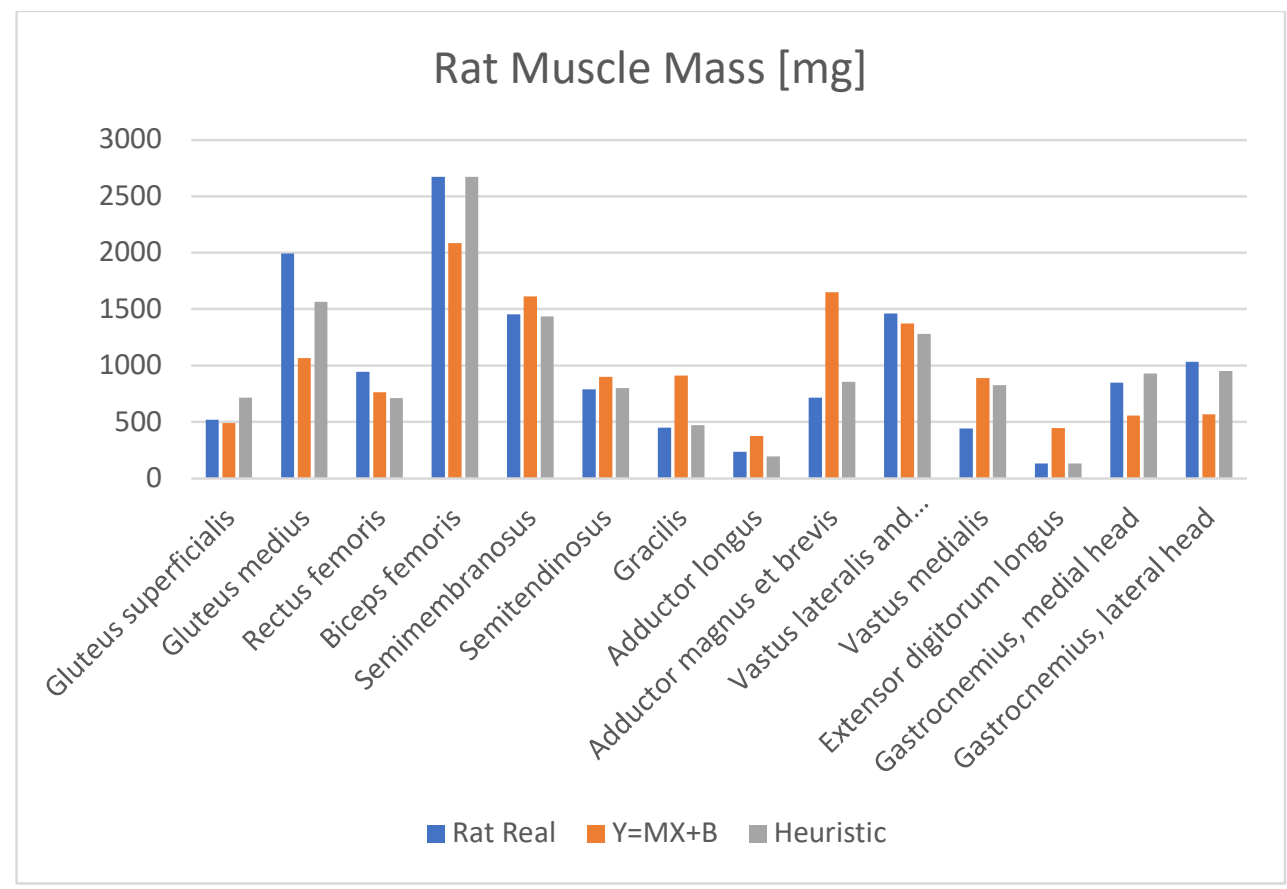

Figure 4.5. The predicted muscle mass values for the rat hindlimb using the heuristic compared to the real rat values.

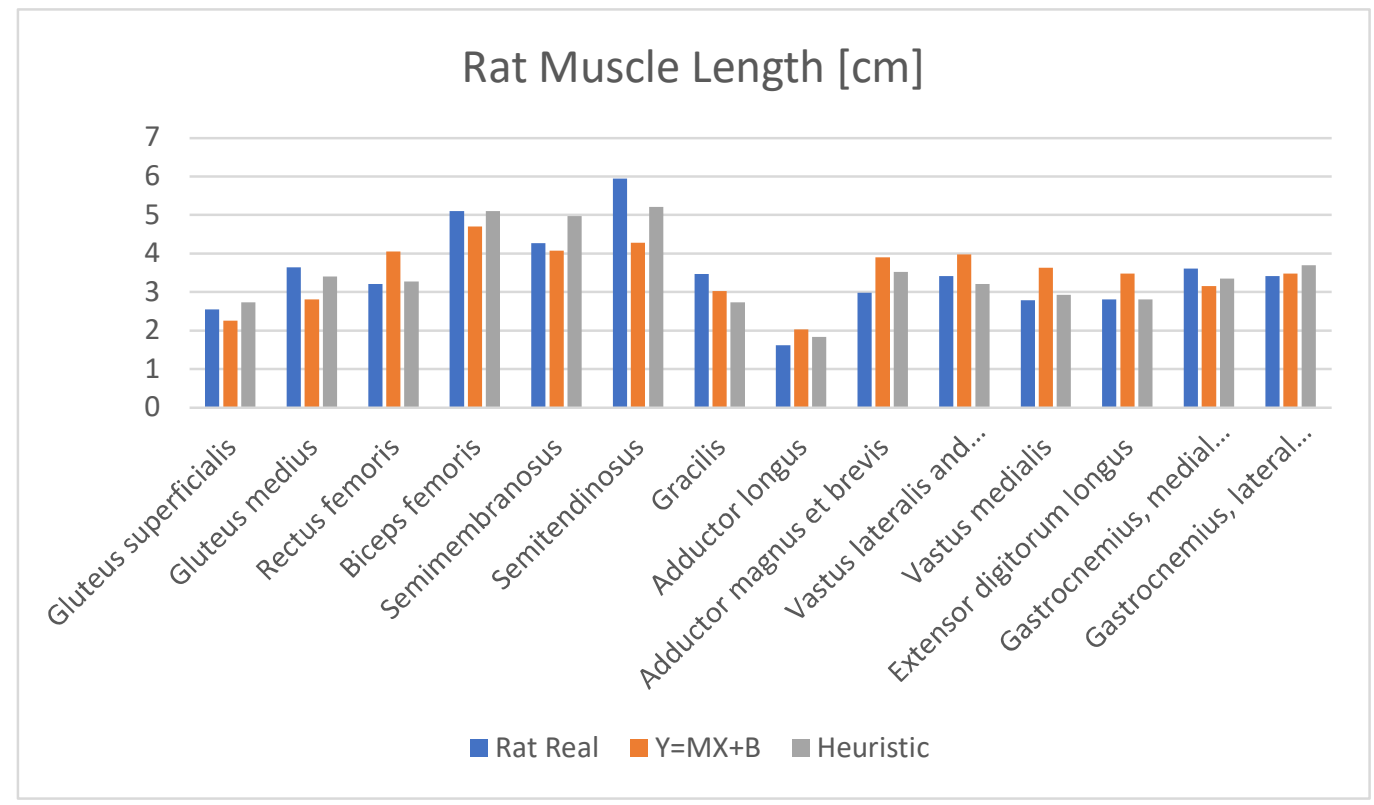

Figure 4.6. The predicted muscle resting length values for the rat hindlimb using the heuristic compared to the real rat values. 


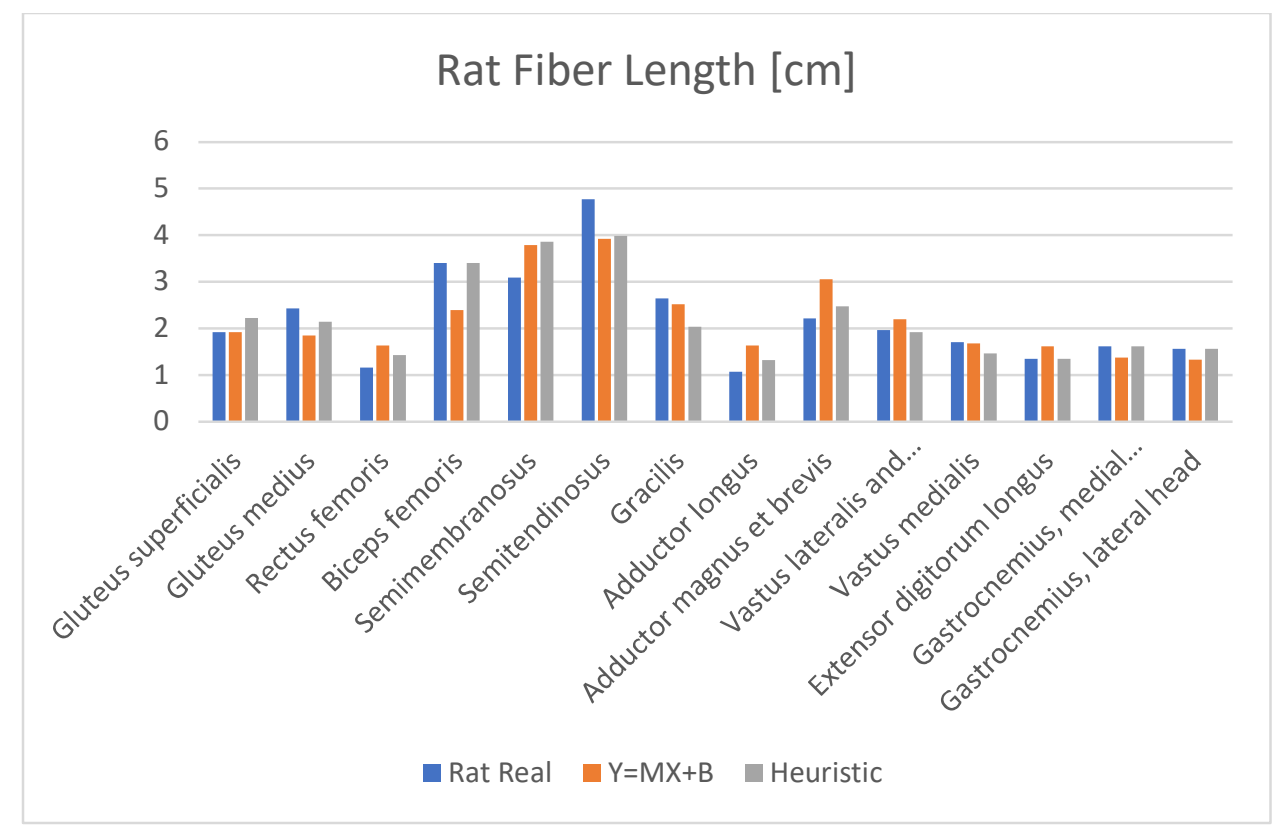

Figure 4.7. The predicted fiber length values for the rat hindlimb using the heuristic compared to the real rat values.

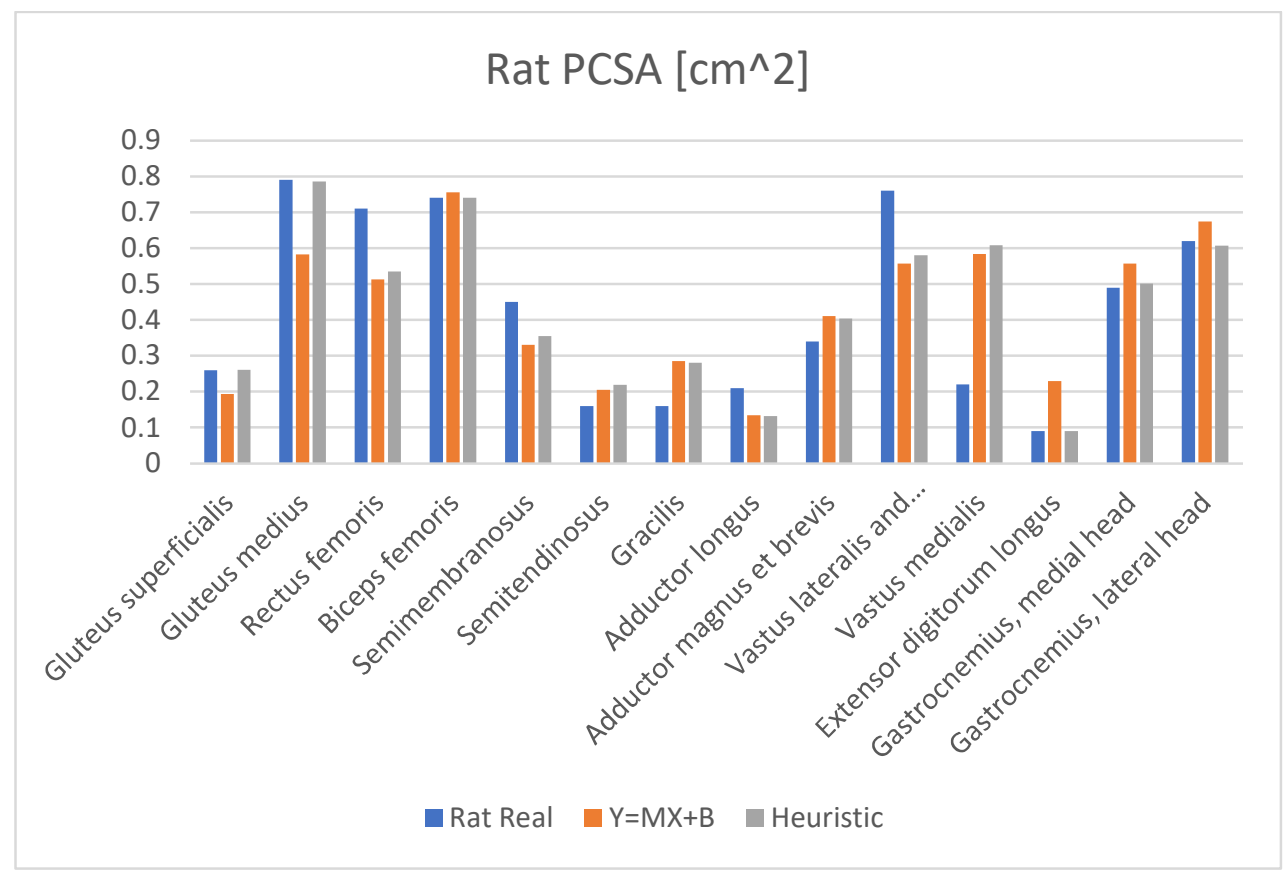

Figure 4.8. The predicted PCSA values for the rat hindlimb using the heuristic compared to the real rat values. 
Since $R^{2}$ values could not be used because the heuristic model is non-linear the percent error for each model was compared.

Table 14. The percent error for each model. Values are represented as mean \pm standard deviation.

\begin{tabular}{lcccc} 
Model & Mass & Muscle Length & Fiber Length & PCSA \\
\hline Linear Model & $59.7 \pm 62.8$ & $18.2 \pm 9.4$ & $21.0 \pm 14.7$ & $45.7 \pm 49.5$ \\
Heuristic Model & $17.4 \pm 22.0$ & $8.8 \pm 6.4$ & $11.9 \pm 9.4$ & $29.9 \pm 45.4$ \\
L - H & 42.3 & 9.4 & 9.1 & 15.7
\end{tabular}

The heuristic model was then used to make rat forelimb predictions.

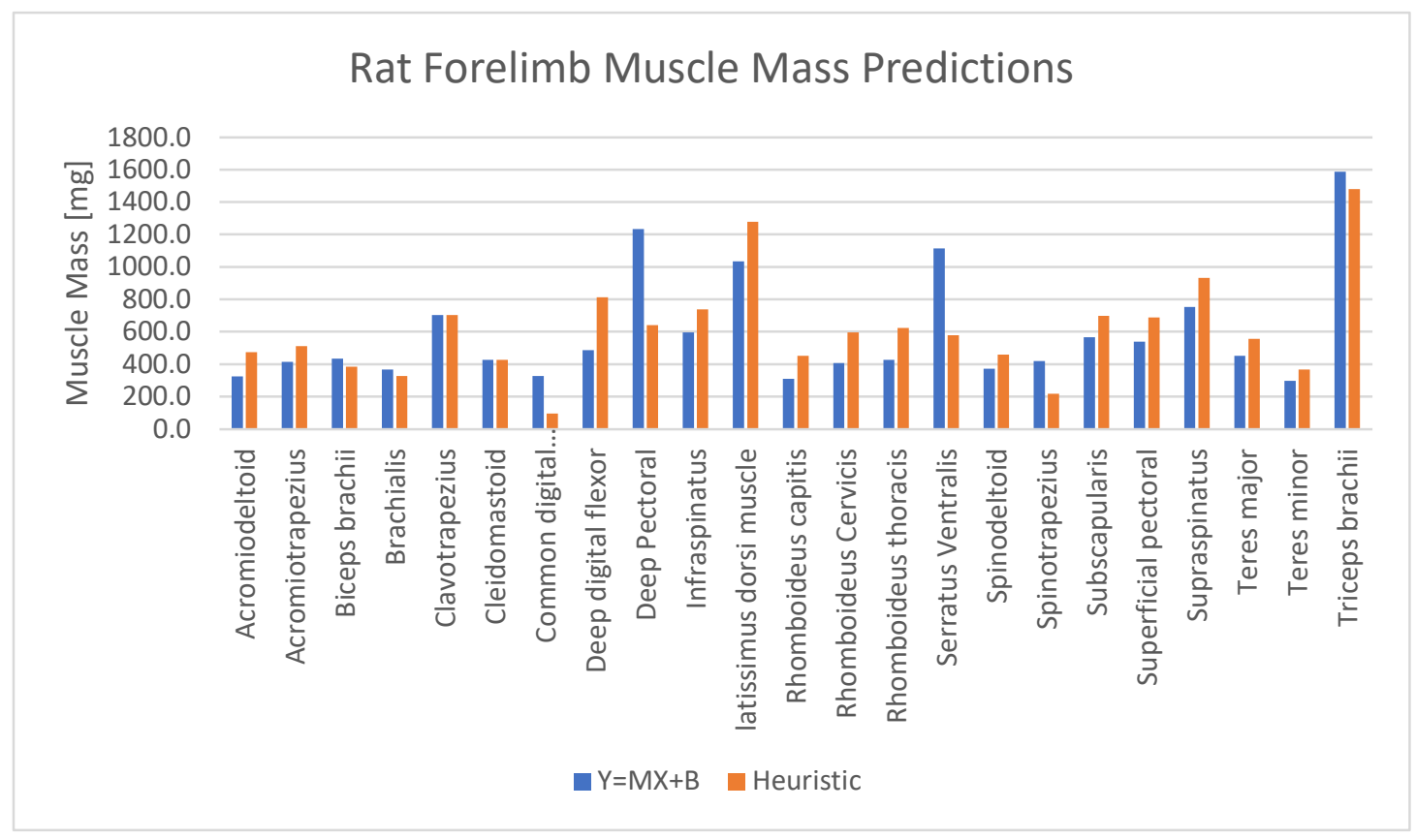

Figure 4.9. The rat forelimb muscle mass predictions from the linear model as well as the heuristic model. 


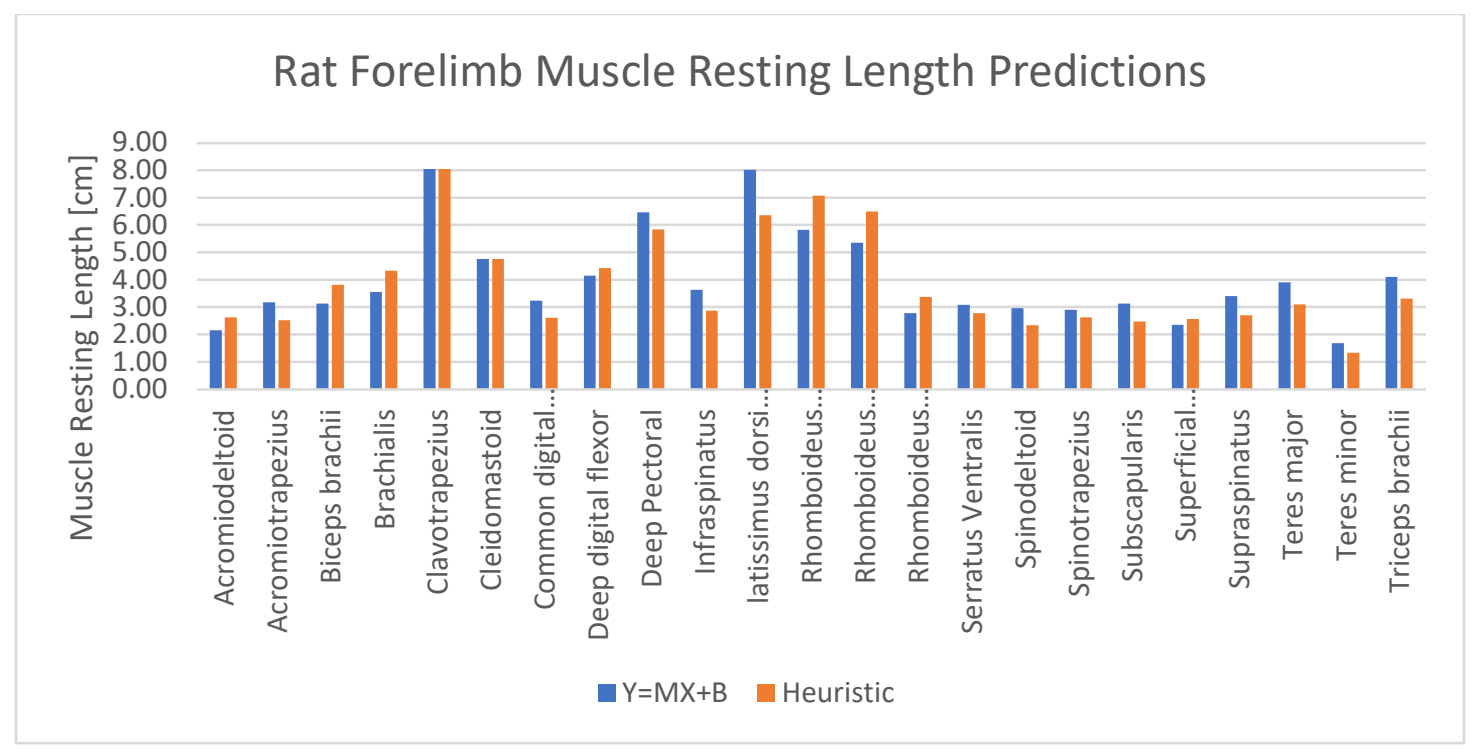

Figure 4.10. The rat forelimb muscle resting length predictions from the linear model as well as the heuristic model.

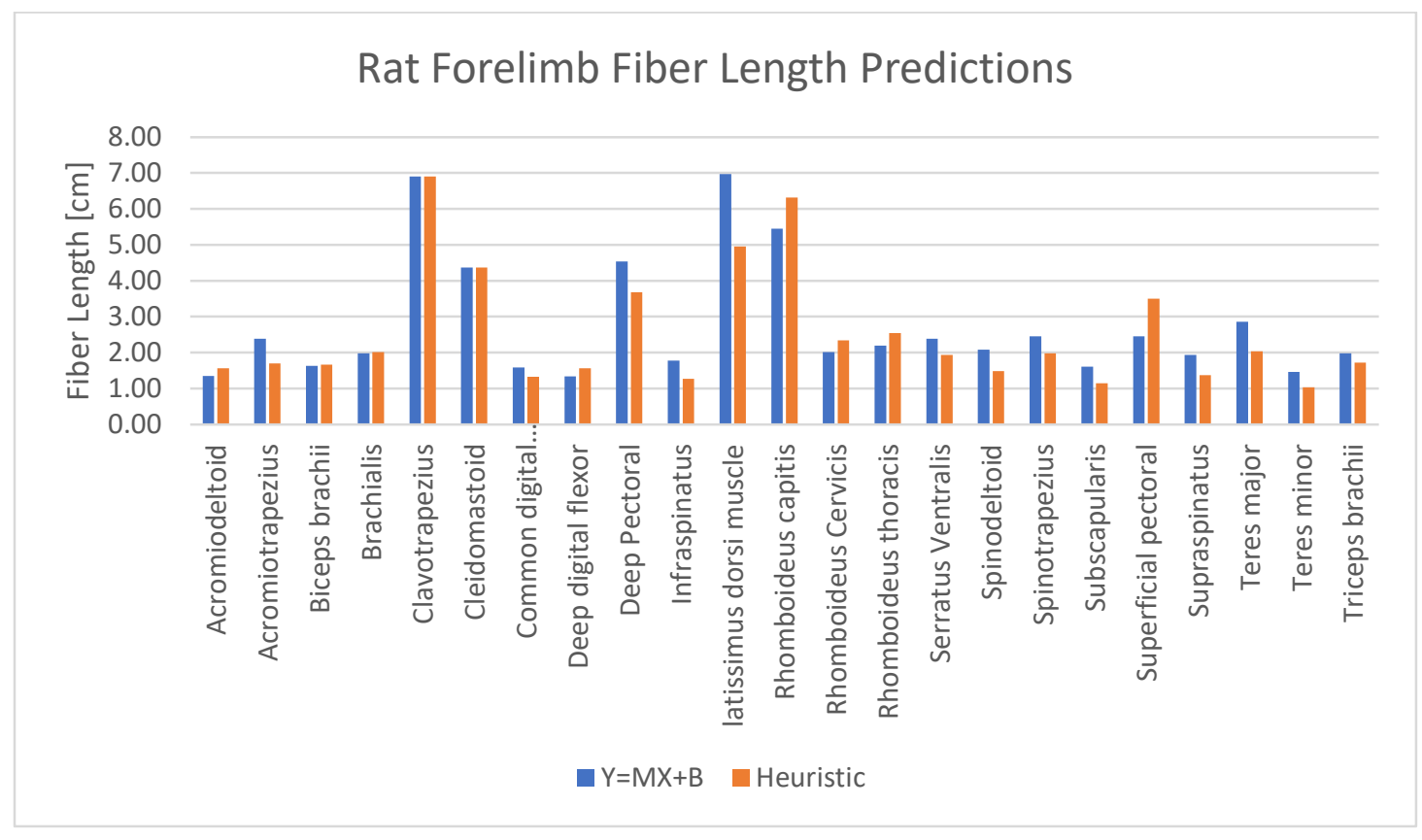

Figure 4.11. The rat forelimb fiber length predictions from the linear model as well as the heuristic model. 


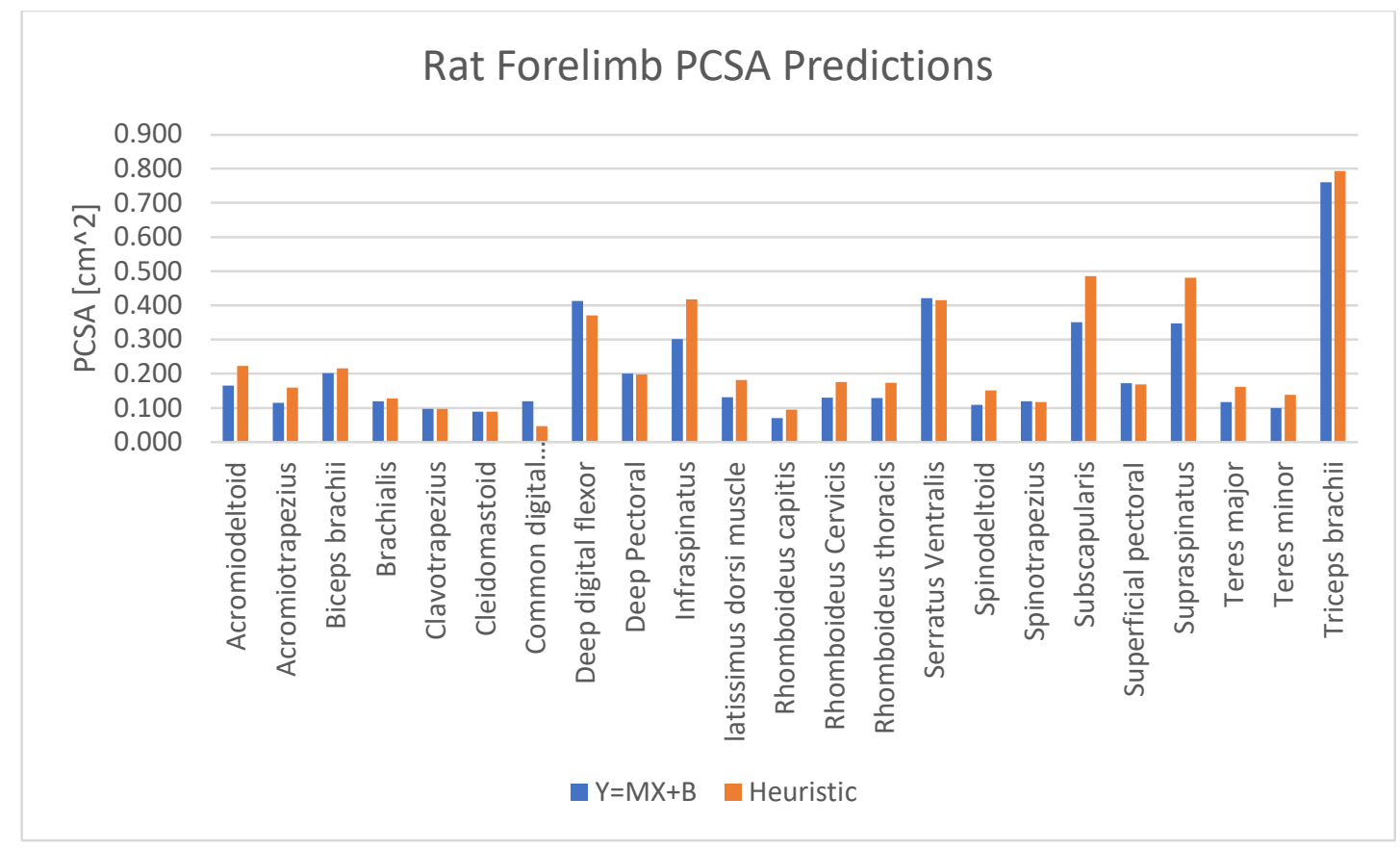

Figure 4.12. The rat forelimb PCSA predictions from the linear model as well as the heuristic model.

\subsubsection{Rat Muscle Fiber Type}

The heuristic method was applied to muscle fiber type using the same classes and approach as the muscle mass, resting length, fiber length, and PCSA.

Table 15. The adjustments needed to transition from dog fast-twitch muscle percentage to rat.

\begin{tabular}{cllc} 
Class & Hindlimb & Forelimb & Adjustment \\
\hline $\mathbf{1}$ & Adduction & Adduction & 1.01 \\
$\mathbf{2}$ & Abduction & Abduction & 1.41 \\
$\mathbf{3}$ & Hip Flexor & Shoulder Flexor & 1.63 \\
$\mathbf{4}$ & Hip Extensor & Shoulder Extensor & 1.36 \\
$\mathbf{5}$ & Knee Flexor & Elbow Flexor & 1.30 \\
$\mathbf{6}$ & Knee Extensor & Elbow Extensor & 1.99 \\
$\mathbf{7}$ & Dorsiflexor & Dorsiflexor & 1.38 \\
$\mathbf{8}$ & Plantar-flexor & Plantar-flexor & 1.77
\end{tabular}




\subsubsection{The Rat Forelimb Muscle Architectural Predictions}

The heuristic model was used to predict forelimb architectural values.

Table 16. Forelimb architecture predicted by the heuristic method.

\begin{tabular}{|c|c|c|c|c|c|}
\hline Muscle Name & $\begin{array}{c}\text { Muscle } \\
\text { Mass [mg] }\end{array}$ & $\begin{array}{c}\text { Resting } \\
\text { Length } \\
{[\mathrm{cm}]}\end{array}$ & $\begin{array}{c}\text { Fiber } \\
\text { Length } \\
{[\mathrm{cm}]}\end{array}$ & $\begin{array}{c}\text { PCSA } \\
{\left[\mathbf{c m}^{2}\right]}\end{array}$ & $\begin{array}{c}\text { Type II } \\
\text { muscle } \\
\text { fiber [\%] }\end{array}$ \\
\hline Acromiodeltoid & 474.35 & 2.62 & 1.57 & 0.22 & 67.8 \\
\hline Acromiotrapezius & 512.06 & 2.52 & 1.70 & 0.16 & 99.0 \\
\hline Biceps brachii & 385.85 & 3.82 & 1.66 & 0.22 & 67.8 \\
\hline Brachialis & 327.75 & 4.33 & 2.01 & 0.13 & 69.1 \\
\hline Clavotrapezius & 703.0 & 8.0 & 6.9 & 0.1 & 62.5 \\
\hline Cleidomastoid & 426.6 & 4.7 & 4.4 & 0.1 & 77.0 \\
\hline Common digital extensor & 96.62 & 2.61 & 1.32 & 0.05 & 99.0 \\
\hline Deep digital flexor & 813.95 & 4.42 & 1.57 & 0.37 & 99.0 \\
\hline Deep Pectoral & 639.91 & 5.83 & 3.68 & 0.20 & 70.6 \\
\hline Infraspinatus & 737.78 & 2.87 & 1.27 & 0.42 & 66.7 \\
\hline latissimus dorsi muscle & 1279.39 & 6.35 & 4.95 & 0.18 & 99.0 \\
\hline Rhomboideus capitis & 452.95 & 7.06 & 6.32 & 0.09 & 65.1 \\
\hline Rhomboideus Cervicis & 596.27 & 6.49 & 2.34 & 0.18 & 44.8 \\
\hline Rhomboideus thoracis & 624.12 & 3.37 & 2.54 & 0.17 & 85.5 \\
\hline Serratus Ventralis & 577.64 & 2.78 & 1.93 & 0.41 & 48.4 \\
\hline Spinodeltoid & 460.05 & 2.34 & 1.48 & 0.15 & 81.4 \\
\hline Spinotrapezius & 217.95 & 2.62 & 1.98 & 0.12 & 63.0 \\
\hline Subscapularis & 698.24 & 2.48 & 1.14 & 0.49 & 91.1 \\
\hline Superficial pectoral & 688.33 & 2.56 & 3.50 & 0.17 & 76.2 \\
\hline Supraspinatus & 931.49 & 2.70 & 1.37 & 0.48 & 87.9 \\
\hline Teres major & 557.03 & 3.09 & 2.03 & 0.16 & 84.6 \\
\hline Teres minor & 366.65 & 1.33 & 1.04 & 0.14 & 99.3 \\
\hline Triceps brachii & 1479.84 & 3.31 & 1.73 & 0.79 & 76.8 \\
\hline
\end{tabular}




\subsubsection{The Tendon Architectural Predictions}

Once the deviation of all the muscles bodies compared to the mean muscle values were determined, the same deviation was applied to the tendons. The following values were predicted for the different tendons.

Table 17. The tendon values used.

\begin{tabular}{lccc} 
Muscle attached to tendon & $\begin{array}{c}\text { Tendon } \\
\text { Length } \\
{[\boldsymbol{\mu m}]}\end{array}$ & $\begin{array}{c}\text { Tendon } \\
\text { Diameter } \\
{[\boldsymbol{\mu m}]}\end{array}$ & $\begin{array}{c}\text { Tendon } \\
\text { ACSA } \\
{[\boldsymbol{\mu m}]^{2}}\end{array}$ \\
\hline Acromiodeltoid & 341.1 & 31.3 & 771.0 \\
\hline Acromiotrapezius & 328.1 & 53.0 & 2204.1 \\
\hline Biceps brachii & 497.1 & 61.7 & 2990.2 \\
\hline Brachialis & 564.4 & 47.4 & 1766.4 \\
\hline Clavotrapezius & 1046.8 & 41.5 & 1350.8 \\
\hline Cleidomastoid & 618.5 & 39.6 & 1233.6 \\
\hline Common digital extensor & 339.8 & 28.7 & 647.5 \\
\hline Deep digital flexor & 575.2 & 80.9 & 5134.0 \\
\hline Deep Pectoral & 759.8 & 59.1 & 2738.8 \\
\hline Infraspinatus & 374.4 & 85.8 & 5785.0 \\
\hline latissimus dorsi muscle & 826.9 & 56.6 & 2514.9 \\
\hline Rhomboideus capitis & 919.9 & 40.8 & 1307.4 \\
\hline Rhomboideus Cervicis & 845.1 & 55.7 & 2437.7 \\
\hline Rhomboideus thoracis & 439.5 & 55.4 & 2410.6 \\
\hline Serratus Ventralis & 361.8 & 85.5 & 5744.2 \\
\hline Spinodeltoid & 305.0 & 51.6 & 2088.2 \\
\hline Spinotrapezius & 341.3 & 45.5 & 1623.7 \\
\hline Subscapularis & 323.0 & 92.5 & 6717.4 \\
\hline Superficial pectoral & 333.2 & 54.5 & 2332.6 \\
\hline Supraspinatus & 351.3 & 92.1 & 6666.3 \\
\hline Teres major & 402.7 & 53.4 & 2236.6 \\
\hline Teres minor & 173.8 & 49.3 & 1907.3 \\
\hline Triceps brachii & 431.2 & 118.2 & 10977.2 \\
\hline & & & \\
\hline
\end{tabular}




\subsection{The Passive and Active Predicted Muscle Parameters for the Rat Forelimb}

The max active contractile force $(A)$ along with the max shortening velocity $(\dot{L})$ and the viscoelastic parameters $\left(k_{s e}, k_{p e}, b\right)$ for the rat forelimb are shown in Table 18.

Table 18. The passive and active muscle parameters predicted for the forelimb.

\begin{tabular}{|c|c|c|c|c|c|}
\hline Muscle Name & $\begin{array}{c}A \\
{[\mathbf{N}]}\end{array}$ & $\begin{array}{c}\dot{L} \\
{[\mathrm{~cm} / \mathbf{s}]}\end{array}$ & $\begin{array}{c}\boldsymbol{k}_{p e} \\
{[\mathbf{N} / \mathbf{m}]}\end{array}$ & $\begin{array}{c}\boldsymbol{k}_{s e} \\
{[\mathbf{N} / \mathbf{m}]}\end{array}$ & $\begin{array}{c}b \\
{[\mathrm{Ns} / \mathbf{m}]}\end{array}$ \\
\hline Acromiodeltoid & 5.0 & 33 & 19.6 & 1130.33 & 16.5 \\
\hline Acromiotrapezius & 3.6 & 40 & 14.6 & 3358.68 & 9.9 \\
\hline Biceps brachii & 4.9 & 49 & 13.1 & 3007.42 & 11.0 \\
\hline Brachialis & 2.9 & 56 & 6.8 & 1564.79 & 5.7 \\
\hline Clavotrapezius & 2.2 & 98 & 2.8 & 645.24 & 2.5 \\
\hline Cleidomastoid & 2.0 & 65 & 4.3 & 997.27 & 3.4 \\
\hline Common digital extensor & 1.1 & 41 & 4.1 & 952.73 & 2.8 \\
\hline Deep digital flexor & 8.3 & 70 & 19.4 & 4462.85 & 13.1 \\
\hline Deep Pectoral & 4.5 & 76 & 7.8 & 1802.20 & 6.4 \\
\hline Infraspinatus & 9.4 & 36 & 33.5 & 7725.63 & 28.5 \\
\hline latissimus dorsi muscle & 4.1 & 101 & 6.6 & 1520.61 & 4.5 \\
\hline Rhomboideus capitis & 2.1 & 88 & 3.1 & 710.60 & 2.7 \\
\hline Rhomboideus Cervicis & 4.0 & 68 & 6.3 & 1442.21 & 6.4 \\
\hline Rhomboideus thoracis & 3.9 & 49 & 11.9 & 2742.27 & 8.8 \\
\hline Serratus Ventralis & 9.3 & 30 & 34.5 & 7938.42 & 34.2 \\
\hline Spinodeltoid & 3.4 & 33 & 14.9 & 3423.41 & 11.3 \\
\hline Spinotrapezius & 2.6 & 32 & 10.3 & 2378.66 & 9.0 \\
\hline Subscapularis & 10.9 & 37 & 45.2 & 10399.01 & 32.1 \\
\hline Superficial pectoral & 3.8 & 35 & 15.2 & 3500.04 & 12.0 \\
\hline Supraspinatus & 10.8 & 40 & 41.2 & 9489.07 & 30.0 \\
\hline Teres major & 3.6 & 45 & 12.1 & 2777.07 & 9.0 \\
\hline Teres minor & 3.1 & 21 & 23.8 & 5485.36 & 16.1 \\
\hline Triceps brachii & 17.8 & 45 & 55.3 & 12729.58 & 43.4 \\
\hline
\end{tabular}




\subsection{The AnimatLab Model}

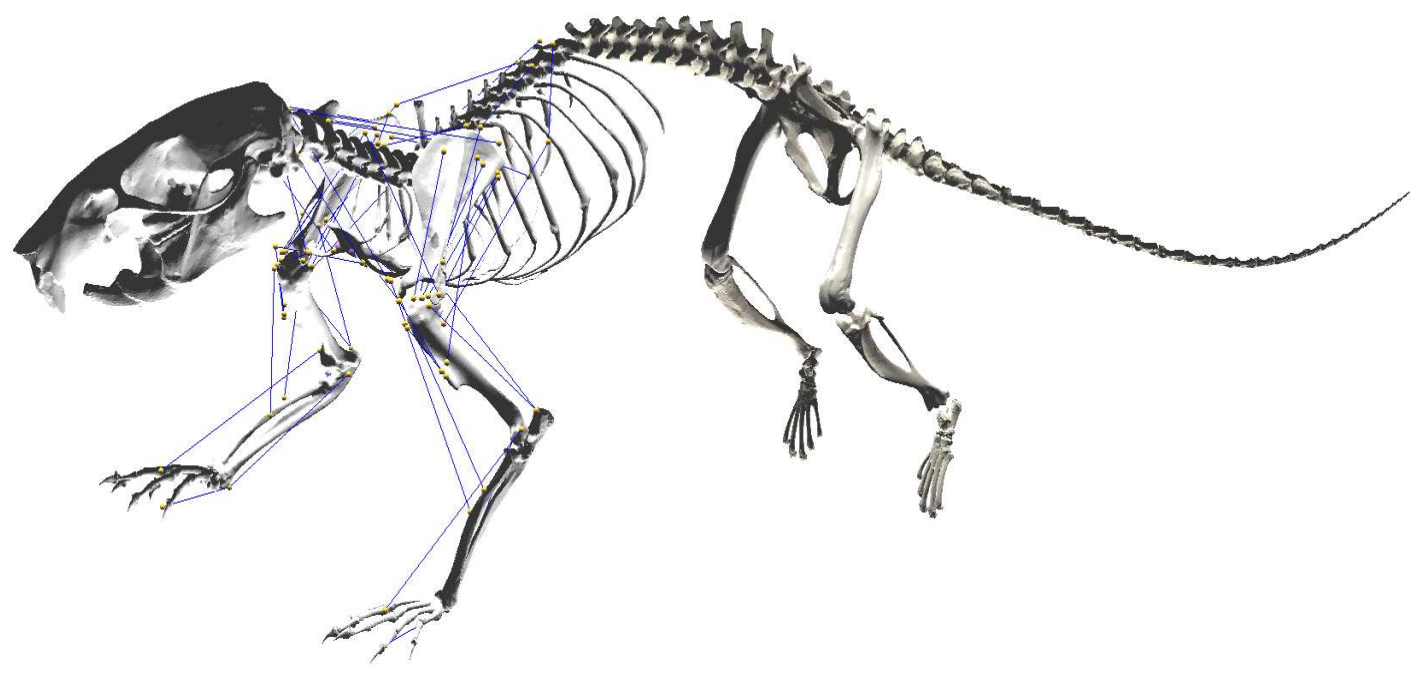

Figure 4.13. The AnimatLab model of the rat. Blue lines represent the muscle-tendon unit.

A video was used to evaluate the AnimatLab model with the real rat. Information for this video can be found in the appendix. 


\section{Chapter 5 Discussion and Future Work}

\subsection{Rat Muscle Architecture}

\subsubsection{Linear Regression}

The low $R^{2}$ values from the linear best fit lines between dog and rat architectural properties, demonstrates there is not a strong linear relationship between the two. However, upon examination of the figures there seemed to be clusters of muscles in two to three distinct groups.

\subsubsection{Using the proportional length approach.}

The challenge of this approach was skeletal data was not available for either the rat or the dog. Therefore, the 'mean dog' lengths were used, and the rat limbs were approximated using the mesh files for the model. However, ultimately this was worse at predicting rat forelimb muscles than simply applying the linear relationship equations. The main issue from this method was the dog data reported by Shahar, was from a "mixed breed" dog and therefore exact proportions could not be determined.

\subsubsection{K-Means Analysis}

After seeing what appeared to be clusters of muscle data from the single linear model, the k-means algorithm identified three distinct clusters. However, the primary issue seemed to be a lack of data. An $80 / 20$ split means each model had at times three inputs to train and one to test. Had the data been an order of magnitude larger, this method may have proved valuable. Additionally, it was difficult to determine why the muscles were clustered the way they were. Upon first glance it seemed the main factor was the 
overall size, however, if the data was orders of magnitude larger, the similarities could be further explored.

\subsubsection{Multiple Linear Regression}

As promising as some of the $\mathrm{R}^{2}$ values are, this is a textbook example of overfitting due to a lack of data. In fact, when these models were used to predict forelimb values after being trained on the hindlimb, often times negative values for mass, resting muscle length, fiber length, and PCSA were predicted. Each model produced coefficients for each input and mass played the smallest role in determining values. This could be due to the fact that dog mass was in considerably larger than the corresponding resting length, fiber length, and PCSA values. The hope was by establishing relationships between the data such as density which was calculated using the muscle mass, resting length, and PCSA that the model would be more effective. This seemed to be effective during the training phase, however, as stated earlier in the end this overfit the model.

\subsubsection{Heuristic Model}

The difference in dog and rat standing posture seemed to be the most plausible explanation for the reason why a linear method could not consistently predict rat architectural values. Unfortunately, due to the severe lack of data, some classes only contained one muscle. Therefore, mean and standard deviation are functionally useless for those classes. However, it was evident that certain classes needed to be bigger or smaller when compared to the linear predicted dog - rat values. These values were then applied to each subsequent class for the rat forelimbs, effectively offsetting the 
linear relationship per class to better represent the real system. When this model was compared to the single linear regression and multiple linear regression model, the heuristic method outperformed both (Figure 4.3 and Table 14). The main reasons were,

1) the multiple linear regression was overfit to the training data and

2) the single linear regression model did not seem to be able to account for a difference in rat posture.

Because the single linear model could not correct for these differences in posture the heuristic method was chosen to offset the rat muscles based on function (adduction, abduction, flexion, extension, etc.).

\subsubsection{Muscle fiber type}

The heuristic method also proved to be the most reliable method for predicting rat forelimb muscle fiber type. The rat muscles had a higher percent of fast twitch fibers than the dog [14] [15]. This could be because the rat moves more quickly relative to its body sixe when compared to larger animals. Some muscle classes required little adjustment, such as muscles used for adduction, whereas fast-twitch muscle fibers in muscles used in the knee/elbow extension were nearly twice as abundant in the rat as they were in the dog. This is believed to be primarily due to the difference in posture. Only fast-twitch muscle fibers needed to be determined since the sum of fast- and slow-twitch muscle fiber type always equals 100\%. Therefore slow-twitch muscles were determined by subtracting the fast-twitch muscle percentage from $100 \%$. 


\subsubsection{Tendon Architecture}

The challenge with tendon architecture was that there was no data available other than the mean length and diameter values for rat hindlimbs [29]. However, having the tendon deviate from the tendon mean in the same way the muscle deviated from the muscle mean, allowed for specialized tendon values which were dependent upon the muscle they were connected to. While this method is not ideal, it does prevent a tendon from having a single unvaried diameter when connected to a muscle that has twice the diameter as the average muscle.

\subsection{Active and Passive Muscle Properties}

Using the Modulus of Elasticity and geometric properties is a tried a true method applied across all of material science to include metals, ceramics, polymers, and woods. Using the same approach for skeletal muscle allows for precise, although the accuracy is still not fully known, predictions. When muscles are similar in shape, fiber type, and pennation angle, they are predicted to have similar values. For example, the acromiotrapezius muscle and teres major muscle have similar mass, resting muscle length, fiber length, and PCSA values. Therefore, these muscles have very similar predicted muscle properties. One of the benefits of this approach is it allows muscles to have predictable values.

\subsection{The AnimatLab Model Performance}

The AnimatLab rat's back, head, and hips were held fixed while the fore and hindlimbs are free to move. Prior to the muscle parameters being added to the model the rat forelimbs would hang in an unnatural position with the scapulas inverting and 
resting on the humerus. Additionally, the limbs would form a straight column when the simulation was run. After the muscle parameters were updated and the simulation began running the rat forelimbs would remain in a crouched, abducted stance very similar to the real rat when it was suspended with its limbs freely hanging at its sides.

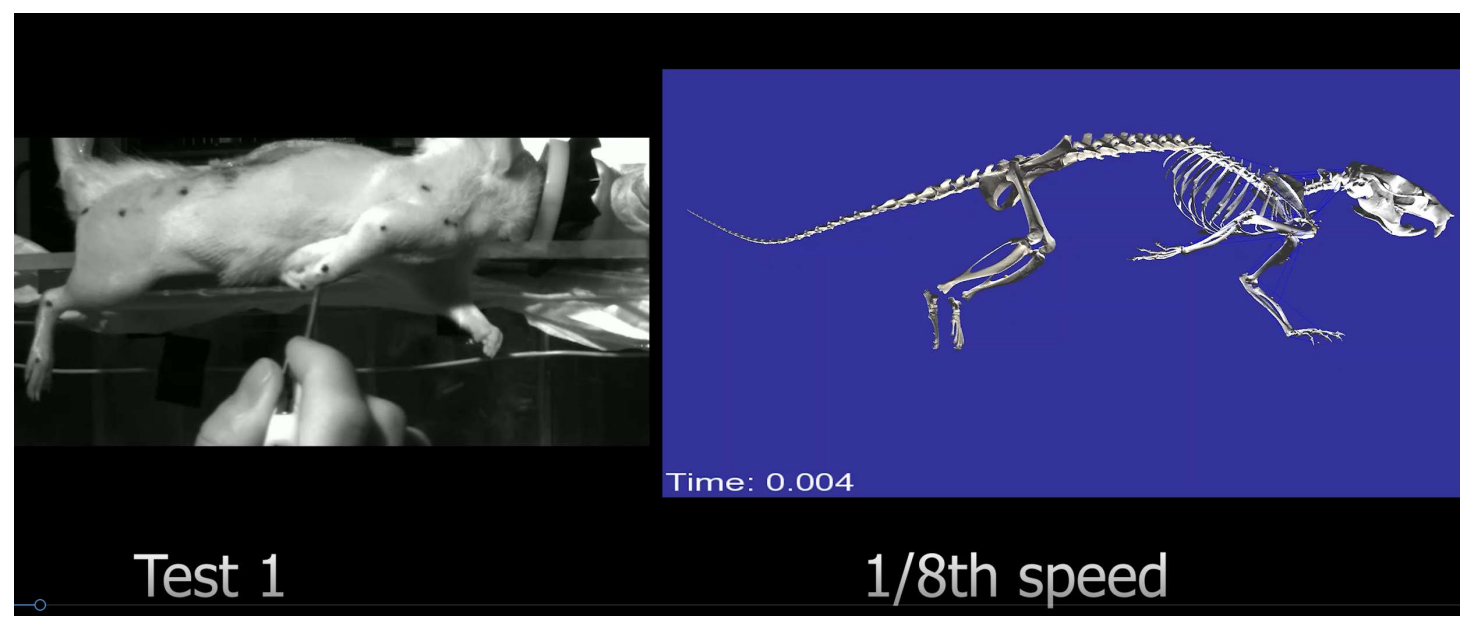

Figure 5.1. A screen shot of the video used to validate the AnimatLab model, showing the initial conditions for test 1.

During the first test, the real rat arm reaches the resting position faster. This could be due to one of two reasons or a combination of the two.

1. The mass of the simulated forelimb was less than the real rat forelimb.

2. The elastic elements of the real rat could be more tight (larger $k_{p e}$ and $k_{s e}$ ) than the simulated model.

The mass of the simulated rat was less than the real system. This is because seven of the 30 forelimb muscles were not included in the simulated rat because they were used to manipulate the phalanges. Therefore, the simulated model is missing the mass of these muscles. Additionally, there was no data on the weight of the skeleton or the rats 
body fat or skin and hair. This would mean the real rat's forelimb would weigh more and would be less effected by any opposing elastic element. Furthermore, the spring constants used in the rat forelimb could be smaller than the real system. However, the damping of the simulated model seems to be very similar to the real rat as upon careful examination both the real rat and the simulated rats forelimb overshoot the final resting position twice.

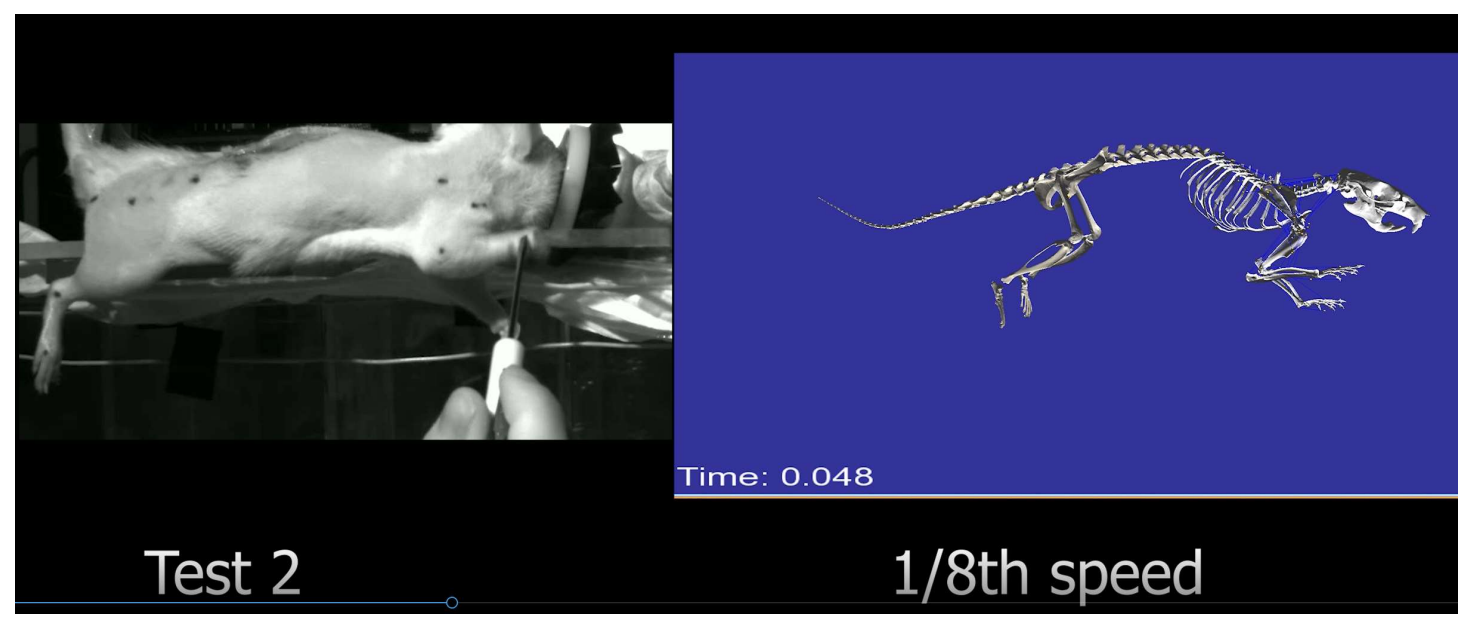

Figure 5.2. A screen shot of the video used to validate the AnimatLab model, showing the initial conditions for test 2.

During the second test, the real rat forelimb reaches the resting position faster. This could be due to three reasons or a combination of the three:

1. The mass of the simulated forelimb was less than the real rat forelimb.

2. The elastic elements of the real rat could be more tight (larger $k_{p e}$ and $k_{s e}$ ) than the simulated model.

3. Muscles could be overdamped, decreasing the velocity of the system. 
As mentioned previously the mass of the forelimb especially the lower portion of the limb was less in the simulated model when compared to the real rat. Additionally, the spring constants for some of the muscles could have been less in the simulated model when compared to the real rat. Finally, the muscles in the forelimb could have a larger damping coefficient $(b)$ than the real rat.

Another challenge of the AnimatLab model is the fact the model does not and cannot account for the effects of the skin, hair, and body fat. Skin has an elastic component to it and at this time skin cannot be added to the AnimatLab model. When looking at the real rat it can be seen that the forelimb begins to distinguish itself from the main body at approximately the elbow (Figure 5.1). Whereas the simulated model's forelimb is distinguished from the body at the sternoclavicular joint. This is because the AnimatLab model does not account for skin. 


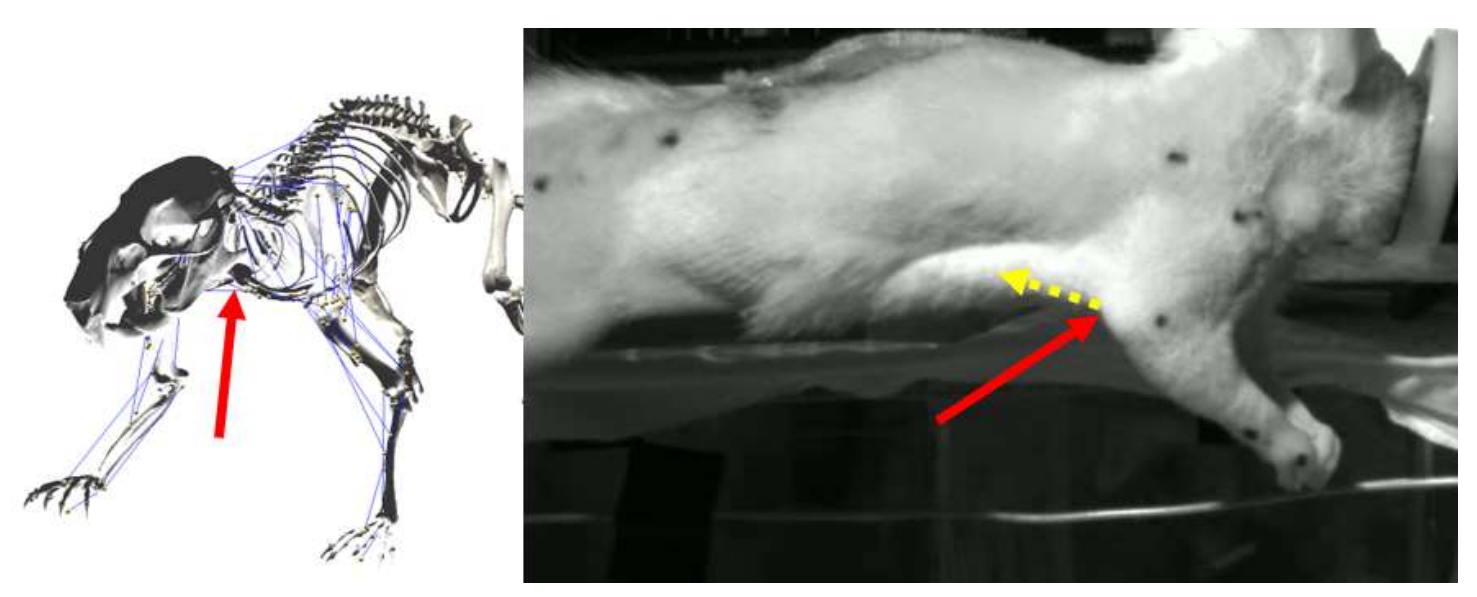

Figure 5.3. The image on the left is of the AnimatLab model and the image on the right is an anesthetized rat. The red arrows indicate where the limb begins to distinguish itself from the main body and the yellow dotted arrow indicated a possible spring force exerted on the forelimb by the skin.

Future work needed for this model is to have a rat preform tests 1 and 2 and to use shearwave ultrasound elastography to measure the spring force and modulus of elasticity of each muscle [35]. Then the rat's architectural data for all the muscles of the fore- and hindlimb, as well as muscle fiber types and tendon architectural data need to be collected. Additionally, the weight and volume of body fat and skin needs to be collected. Muscle properties can then be predicted with the real values obtained to determine the accuracy of the predictive muscle properties model. The AnimatLab model can be updated to reflect the real values and the AnimatLab model can then preform Test 1 and Test 2 and the results can be compared with the rat the data was obtained from. When the model is based off of and compared to a single rat the real model accuracy can be evaluated. Currently the AnimatLab model is based off a blend of different rat and dog data and then tested against a different rat. This was due to a lack of data. 


\subsection{Conclusion}

The main roadblock for this research was the lack of available data for the rat forelimb. This proved to be one of the most challenging and time-consuming aspects of the overall research. It also provides the most uncertainty for the model. This is because the viscoelastic parameters are determined using the predicted architectural data. Then the AnimatLab model is built using the predicted viscoelastic properties. Fundamentally the AnimatLab is restricted in its accuracy by the accuracy of the rat architectural data. Additionally, on the road to developing a biomechanical model of the rat forelimb, theoretically determining the viscoelastic parameters as well as the active contractile force, and contractile velocities were especially difficult given that a unified method is not established. The methods for the model proposed in this work are based off tried and true material science methods. More work needs to be done to gather more data on the modulus of elasticity for skeletal muscle and tendons.

Similarly, more data is needed to determine the effects of muscle fiber type with respect to force contraction and viscous damping. While there may be many factors in determining how skeletal muscle will behave, I would argue we should expect this. Just as in material science where for isotropic materials the properties such as Young's Modulus, tensile yield and ultimate strength are greatly altered depending on the how the material was manufactured (hot-rolled, cold rolled, cast, etc.) or what type of alloy it is, so there is also variety in skeletal muscle depending on pennation angle, muscle fiber type and overall muscle size. This great variety of materials has been studied for nonbiological systems since the iron age and we now have enough data to be able to 
predict how each material will behave. Furthermore, even materials whose properties are highly anisotropic are broken down and studied until we are able to make predictions. In this same way, skeletal muscle needs to be studied and predictive models built from fundamental truths, such as sarcomeres are the force generator and pennation angle affects the behavior of the muscle. Once enough data has been collected and all the parameters have been factored, the models established in this work can be refined and more accurate predictions can be made. One of the challenges is that many of the systems have been experimentally shown to be non-linear. This provides another layer of complexity. However, this research proposes a model to develop the muscle properties using linear relationships with the understanding the results will be approximate and deviation from the real non-linear systems in inherent. 


\section{References}

[1] C. M. Eng, L. H. Smallwood, M. P. Rainiero, M. Lahey, S. R. Ward and R. L. Lieber, "Scaling of muscle architecture and fiber types in the rat hindlimb," The Journal of Experimental Biology, vol. 211, pp. 2336-2345, 2008.

[2] R. Shahar and J. Milgram, "Morphometric and anatomic study of the hind limb of a dog," American Journal of Veterinary Research, vol. 62, pp. 928-933, 2001.

[3] R. Shahar and J. Milgram, "Morphometric and Anatomic Study of the Forelimb of the Dog," Journal of Morphology, vol. 263, pp. 107-117, 2005.

[4] A. J. Hunt, N. S. Szczecinski, E. Andrada, M. Fischer and R. D. Quinn, "Using Animal Data and Neural Dynamics to Reverse Engineer a Neuromechanical Rat Model," Biomimetic and Biohybrid Systems. Living Machines 2015. Lecture Notes in Computer Science, vol. 9222, 2015.

[5] R. M. Alexander and H. C. Bennet-Clark, "Storage of elastic strain energy in muscle and other tissues," Nature, vol. 265, pp. 114-177, 1977.

[6] A. Lai, A. G. Schache, Y.-C. Lin and M. G. Pandy, "Tendon elastic strain energy in the human ankle plantar-flexors and its role with increased running speed," Journal of Experimental Biology, vol. 217, pp. 3159-3168, 2014.

[7] E. K. Moo and W. Herzog, "Single sarcomere contraction dynamics in a whole muscle," Scientific Reports, vol. 8, 2018.

[8] H. Huxley and J. Hanson, "Changes in the Cross-Striations of Muscle during Contraction and Stretch and their Structural Interpretation," Nature, vol. 173, pp. 973-976, 1954.

[9] A. F. Huxley and R. Niedergerke, "Structural Changes in Muscle During Contraction: Interference Microscopy of Living Muscle Fibres," Nature, vol. 173, pp. 971-973, 1954.

[10] E. Aziz, E. L. Brainerd and T. J. Roberts, "Variable gearing in pennate muscles," Proceedings of the National Academy of Sciences of the United States of America, vol. 105, pp. 1745-1750, 2008.

[11] S. Schiaffino and C. Reggiani, "Fiber types in mammalian skeletal muscles," Physiological Review, vol. 91, pp. 1447-1531, 2011. 
[12] J.-P. Rospars and N. Meyer-Vernet, "Force per cross-sectional area from molecules to muscles: a general property of biological motors," Royal Society Open Source, vol. 3, no. 7, 2016.

[13] U. S. University, "Structural Factors - Muscle Fiber Type," Utah State University, [Online]. Available: https://usu.instructure.com/courses/422916/pages/structural-factors-musclefiber-type. [Accessed 2505 2021].

[14] R. B. Armstrong and R. O. Phelps, "Muscle fiber type composition of the rat hindlimb," American Journal of Anatomy, vol. 171, no. 3, pp. 259-272, 1984.

[15] R. B. Armstrong, C. W. Saubert IV, H. J. Seeherman and C. R. Taylor, "Distribution of Fiber Types in Locomotory Muscles of Dogs," The American Journal of Anatomy, vol. 163, pp. 87-98, 1982.

[16] W. Scott, J. Stevens and S. A. Binder-Macleod, "Human Skeletal Muscle Fiber Type Classifications," Physical Therapy, vol. 81, pp. 1810-1816, 2001.

[17] K. W. Ranatunga, "The force-velocity relation of rat fast- and slow-twitch muscles examined at different temperatures," The Journal of Physiology, vol. 351, pp. 517-529, 1984.

[18] E. Briese, "Normal Body Temperature of Rats: The Setpoint Controversy," Neuroscience and Biobehavioral Reviews, vol. 22, pp. 427-436, 1998.

[19] R. D. Herbert and S. C. Gandevia, "The passive mechanical properties of muscle," Journal of Applied Physiology, vol. 126, pp. 1442-1444, 2019.

[20] T. J. Roberts, "Contribution of elastic tissues to the mechanics and energetics of muscle function during movement," Journal of Experimental Biology, vol. 219, pp. 266-275, 2016.

[21] C. N. Maganaris and J. P. Paul, "In vivo human tendon mechanical properties," The Journal of Physiology, 2004.

[22] V. Kovanen, H. Suominen and E. Heikkinen, "Mechanical Properties of Fast and Slow Skeletal Muscle with Special Reference to Collagen and Endurance Training," Journal of Biomechanics, vol. 17, pp. 725-735, 1984.

[23] A. V. Hill, "The Heat of Shortening and the Dynamic Constants of Muscle," The Royal Society, vol. 126, no. 843, 1938. 
[24] H. Maas and P. A. Huijing, "Synergistic and antagonistic interactions in the rat forelimb: acute effects of coactivation," Journal of Applied Physiology, vol. 107, pp. 1453-1462, 2009.

[25] J. P. Charles, O. Cappellari, A. J. Spence, J. R. Hutchinson and D. J. Wells, "Musculoskeletal Geometry, Muscle Architecture and Functional Specialisations of the Mouse Hindlimb," Plos One, 2016.

[26] W. L. Johnson, D. L. Jindrich, R. R. Roy and V. R. Edgerton, "A threedimensional model of the rat hindlimb: musculoskeletal geometry and muscle moment arms," J Biomech., 2008.

[27] W. L. Johnson, L. D. Jindrich, H. Zhong, R. R. Roy and V. R. Edgerton, "Application of a Rat Hindlimb Model: A Prediction of Force Spaces Reachable Through Stimulation of Nerve Fascicles," IEE Trans Biomed Eng., 2011.

[28] P. Negredo, J.-L. L. Rivero, B. González, A. Ramón-Cueto and R. Manso, "Slow- and fast-twitch rat hind limb skeletal muscle," The Journal of Physiology, vol. 10, pp. 2593-2610, 2008.

[29] J. Zelená and T. Soukup, "The in-series and in-parallel components in rat hindlimb tendon organs," Neuroscience, vol. 9, pp. 899-910, 1983.

[30] D. Grimm, "Science Mag," Science, 12 Jan 2021. [Online]. Available: https://www.sciencemag.org/news/2021/01/how-many-mice-and-rats-are-usedus-labs-controversial-study-says-more-100-million. [Accessed 12 June 2021].

[31] H. Stark, M. S. Fischer, A. Hunt, F. Young, R. Quinn and E. Andrada, "A threedimensional musculoskeletal model of the dog," Scientific Report, vol. 11, 2021.

[32] M. Fischer, Dogs in Motion (Hunde in Bewegung), Dortmund: VDH Service GmbH, 2011.

[33] A. A. Biewener, "Scaling Body Support in Mammals: Limb Posture and Muscle Mechanics," Science, vol. 245, pp. 45-48, 1989.

[34] D. K. Riskin, C. J. Kendall and J. W. Hermanson, "The crouching of the shrew: Mechanical consequences of limb posture in small mammals," PeerJ, vol. 4, p. e2131, 2016.

[35] Y. N. Feng, Y. P. Li, C. L. Liu and Z. J. Zhang, "Assessing the elastic properties of skeletal muscle and tendon using shearwave ultrasound elastography and MyotonPRO," Nature, 2018. 
[36] C. Then, T. J. Vogl and G. Silber, "Method for characterizing viscoelasticity of human gluteal tissue," Journal of Biomechanics, vol. 45, pp. 1252-1258, 2012.

[37] P. L. Powell, R. R. Roy, P. Kanim, M. A. Bello and V. R. Edgerton, "Predictability of skeletal muscle tension from architectural determinations in guinea pig hindlimbs," Journal of Applied Physiology: Respiratory, Environmental and Exercise Physiology, vol. 57, pp. 1715-1721, 1984.

[38] S. Thallera and H. Wagner, "The relation between Hill's equation and individual muscle properties," Journal of Theoretical Biology, vol. 231, no. 3, 2004.

[39] F. E. Zajac, "Muscle and Tendon: Properties, Models, Scaling, and Application to Biomechanics and Motor Control," Critical Reviews in Biomedical Engineering, vol. 17, pp. 359-411, 1989.

[40] F. Young, A. J. Hunt, H. J. Chiel and R. D. Quinn, "Using Animatlab for Neuromechanical Analysis: Linear Hill Parameter Calculation," Conference on Biomimetic and Biohybrid Systems Living Machines 2020: Biomimetic and Biohybrid Systems, pp. 402-414, 2020.

[41] F. Young, C. Rode, A. Hunt and R. Quinn, "Analyzing Moment Arm Profiles in a Full-Muscle Rat Hindlimb Model," Biomimetics, vol. 4, 2019.

[42] S. Vivekanandan, D. S. Emmanuel and R. S. Saluja, "Modelling of gastrocnemius muscle using Hill's equation in COMSOL Multiphysics 4.0a," International Journal of Computer Science Issues, vol. 9, no. 3, pp. 396-400, 2012.

[43] L. Toniolo, L. Maccatrozzo, M. Patruno, E. Pavan, F. Caliaro, R. Rossi, C. Rinaldi, M. Canepari, C. Reggiani and F. Mascarello, "Fiber types in canine muscles: myosin isoform expression and functional characterization," American Journal of Physiology-Cell Physiology, vol. 292, pp. C1915-C1926, 2007.

[44] M. Tian, P. D. Hoang, S. C. Gandevia, R. D. Herbert and L. E. Bilston, "Viscous elements have little impact on measured passive length-tension properties of human gastrocnemius muscle-tendon units in vivo," Journal of Biomechanics, vol. 44, pp. 1334-1339, 2011.

[45] A. K. Thota, S. C. Watson, E. Knapp, B. Thompson and R. Jung, "Neuromechanical Control of Locomotion in the Rat," Journal of Neurotrauma, vol. 22, pp. 442-465, 2005. 
[46] R. Shahar and L. Banks-Sills, "Biomechanical Analysis of the Canine Hind Limb: Calculation of Forces During Three-legged Stance," The Veterinary Journal, vol. 163, pp. 240-250, 2002.

[47] C. Y. Seow, "Hill's equation of muscle performance and its hidden insight on molecular mechanisms," The Journal of General Physiology, vol. 142, pp. 561$573,2013$.

[48] S. Schleifenbaum, M. Schmidt, R. Möbius, T. Wolfskämpf, C. Schröder, R. Grunert, N. Hammer and T. Prietzel, "Load and failure behavior of human muscle samples in the context of proximal femur," BMC Musculoskeletal Disorders, vol. 17, 2016.

[49] E. D. Ryan, T. J. Herda, P. B. Costa, J. M. Defreitas, T. W. Beck, J. R. Stout and J. T. Cramer, "Passive properties of the muscle-tendon unit: The influence of muscle cross-sectional area," Muscle \& Nerve, vol. 39, no. 2, pp. 227-229, 2009.

[50] F. Romero and F. J. Alonso, "A comparison among different Hill-type contraction dynamics formulations for muscle force estimation," Mechanical Sciences, vol. 7, no. 1, 2016.

[51] C. Reggiani and S. Schiaffino, "Muscle hypertrophy and muscle strength: dependent or independent variables? A provocative review," European Journal of Translational Myology, vol. 30, 2020.

[52] P. M. Prodinger, P. Foehr, D. Bürklein, O. Bissinger, H. Pilge, K. Kreutez, R. v. Eisenhart-Rothe and T. Tischer, "Whole bone testing in small animals: systematic characterization of the mechanical properties of different rodent bones available for rat fracture models," European Journal of Medical Research, vol. 23, 2018.

[53] C. A. Phillips, D. W. Repperger, A. T. Neidhard-Doll and D. B. Reynolds, "Biomimetic model of skeletal muscle isometric contraction: I. an energeticviscoelastic model for the skeletal muscle isometric force twitch," Computers in Biology and Medicine, vol. 34, pp. 307-322, 2004.

[54] M. A. Pellegrino, M. Canepari, R. Rossi, G. D'Antona, C. Reggiani and R. Bottinelli, "Orthologous myosin isoforms and scaling of shortening velocity with body size in mouse, rat, rabbit and human muscles," The Journal of Physiology, vol. 546, no. 3, pp. 677-689, 2003. 
[55] I. Ogneva, D. V. Lebedev and B. S. Shenkman, "Transversal Stiffness and Young's Modulus of Single Fibers from Rat Soleus Muscle Probed by Atomic Force Microscopy," Biophysical Journal, vol. 98, pp. 418-424, 2010.

[56] T. A. McMahon, "Mechanics of Locomotion," The International Journal of Robotics Research, vol. 3, 1984.

[57] C. P. McGowan, R. R. Neptune and W. Herzog, "A phenomenological model and validation of shortening-induced force depression during muscle contractions," Journal of Biomechanics, vol. 43, pp. 449-454, 2010.

[58] M. A. Mathewson, M. A. Chapman, E. R. Hentzen, J. Fridén and R. L. Lieber, "Anatomical, architectural, and biochemical diversity of the murine forelimb muscles," Journal of Anatomy, vol. 221, pp. 443-451, 2012.

[59] R. B. Martin, D. B. Burr, N. A. Sharkey and D. P. Fyhrie, Skeletal Tissue Mechanics, Springer, 2015.

[60] C. N. Maganaris and M. V. Narici, Tendon Injuries - Basic Science and Clinical Medicine, Springer, 2005.

[61] C. N. Maganaris, "Force-length characteristics of in vivo human skeletal muscle," Acta Physiologica Scandinavica, vol. 172, pp. 279-85, 2001.

[62] H. Maas and P. A. Huijing, "Synergistic and antagonistic interactions in the rat forelimb: acute effects of coactivation," Journal of Applied Physiology, vol. 107, pp. 1453-1462, 2009.

[63] D. C. Lin and W. Z. Rymer, "Damping actions of the neuromuscular system with inertial loads: human flexor pollicis longus muscle," Journal of Neurophysiology, vol. 85, pp. 1059-1066, 2001.

[64] R. L. Lieber and S. R. Ward, "Skeletal muscle design to meet functional demands," Philosophical Transactions of the Royal Society B, Biological Series, vol. 366, pp. 1466-1476, 2011.

[65] B. C. W. Kot, Z. J. Zhang, A. W. C. Lee, V. Y. F. Leung and S. N. Fu, "Elastic Modulus of Muscle and Tendon with Shear Wave Ultrasound Elastography: Variations with Different Technical Settings," PLoS ONE, 2012.

[66] K. Jovanovic, J. Vranic and N. Miljkovic, "Hill's and Huxley's Muscle Models Tools for Simulations in Biomechanics," Serbian Journal of Electrical Engineering, vol. 12, pp. 53-67, 2015. 
[67] M. Ilton, M. S. Bhamla, X. Ma, S. M. Cox, L. L. Fitchett, Y. Kim, J.-S. Koh, D. Krishnamurthy, C.-Y. Kuo, F. Z. Temel, A. J. Crosby, M. Prakash, G. P. Sutton, R. J. Wood, E. Azizi, S. Bergbreiter and S. N. Patek, "The principles of cascading power limits in small, fast biological and engineered systems," Science, vol. 360, 2018.

[68] B. I. Hyland and V. Jordan, "Muscle activity during forelimb reaching movements in rats," Behavioural Brain Research, vol. 85, no. 2, pp. 175-186, 1997.

[69] C. Hunter and E. J. Clegg, "Changes in skeletal proportions of the rat in response to hypoxic stress," Journal of Anatomy, vol. 144, pp. 201-219, 1973.

[70] A. Hunt, M. Schmidt, M. Fischer and R. Quinn, "A biologically based neural system coordinates the joints and legs of a tetrapod," Bioinspiration \& Biomimetics, vol. 10, 2005.

[71] M. G. Hoy and R. F. Zernicke, "Modulation of limb dynamics in the swing phase of locomotion," Journal of Biomechanics, vol. 18, pp. 4-60, 1985.

[72] J. W. Holmes, "Teaching from classic papers: Hill's model of muscle contraction," Advances in Physiological Education, vol. 30, no. 2, pp. 67-72, 2006.

[73] D. D. Hatcher and A. R. Luff, "Force-Velocity Properties of Fast-Twitch and Slow-Twitch Muscles of the Kitten," The Journal of Physiology, vol. 367, pp. 377-385, 1985.

[74] S. M. Greising, H. M. Gransee, C. B. Mantilla and G. C. Sieck, "Systems biology of skeletal muscle: fiber type as an organizing principle," Wiley Interdisciplinary Reviews: Systems Biology and Medicine, vol. 4, pp. 457-473, 2013.

[75] E. C. Greene, "Anatomy of the Rat," American Philosophical Society, vol. 27 (1935), p. 370, 1955.

[76] P. D. Gollnick, B. Sjödin, J. Karlsson, E. Jansson and B. Saltin, "Human Soleus Muscle: A Comparison of Fiber Composition and Enzyme Activities with Other Leg Muscles," Pflügers Archiv - European Journal of Physiology, vol. 348, pp. 247-255, 1974.

[77] T. Fukunaga, R. R. Roy, F. G. Shellock, J. A. Hodgson, M. K. Day, P. L. Lee, H. Kwong-Fu and V. R. Edgerton, "Physiological cross-sectional area of human leg 
muscles based on magnetic resonance imaging," Journal of Orthopaedic Research, vol. 10, no. 6, pp. 926-934, 1992.

[78] M. S. Fischer, N. Schilling, M. Schmidt, D. Haarhaus and H. Witte, "Basic limb kinematics of small therian mammals," The Journal of Experimental Biology, vol. 205, pp. 1315-1338, 2002.

[79] W. O. Fenn and B. S. Marsh, "Muscular force at different speeds of shortening," The Journal of Physiology, vol. 85, pp. 277-297, 1935.

[80] R. Close, "Dynamic Properties of Mammalian Skeletal Muscle," The Journal of Physiology, vol. 52, pp. 129-197, 1972.

[81] R. Close, "Dynamic properties of fast and slow skeletal muscle of the ray during development," The Journal of Physiology, vol. 173, pp. 74-95, 1964.

[82] M. F. Bonnan, J. Shulman, R. Varadharajan, C. Gilbert, M. Wilkes, A. Horner and E. Brainerd, "Forelimb Kinematics of Rats Using XROMM, with Implications for Small Eutherians and Their Fossil Relatives," PLoS ONE, vol. 31, pp. 122-132, 2016.

[83] E. Andrada, L. Reinhardt, K. Lucas and M. Fischer, "Three-dimensional inverse dynamics of the forelimb of Beagles at a walk and trot," American Journal of Veterinary Research, vol. 78, pp. 804-817, 2017.

[84] R. M. Alexander, "The maximum forces exerted by animals," Journal of Experimental Biology, pp. 231-238, 1985.

[85] "Human skeletal muscle: transition between fast and slow fibre types," Pflugers Archiv: European Journal of Physiology, vol. 461, pp. 537-543, 2011.

[86] J. M. Metzger and R. L. Moss, "Effects of tension and stiffness due to reduced $\mathrm{pH}$ in mammalian fast- and slow-twitch skinned skeletal muscle fibres.," The Journal of Physiology, vol. 428, pp. 737-750, 1990.

[87] A. Hunt, N. Szczecinski and R. Quinn, "Development and Training of a Neural Controller for Hind Leg Walking in a Dog Robot," Frontiers in Neurorobotics, vol. 11, 2017. 


\section{Appendix Supplemental File Details}

Supplemental file - video file comparison of the real and simulated rat.

Title: Josh's Thesis Rat Passive Parameter Validation Video.avi

File Type: Audio Video Interleave (AVI) File

Size: $7,356 \mathrm{~KB}$

Required application software: AVI viewing software such as Windows media player or VLC media player.

Special hardware requirements: None. 